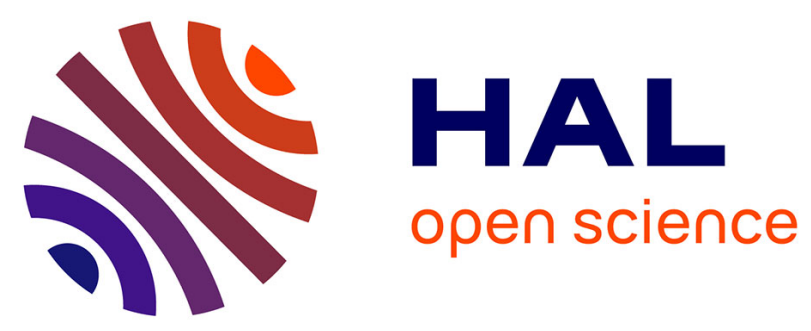

\title{
Un gisement châtelperronien de plein air dans le Bassin parisien : les Bossats à Ormesson (Seine-et-Marne)
}

Pierre Bodu, Hélène Salomon, Jessica Lacarrière, Michael Baillet, Michèle Ballinger, Henri-Georges Naton, Isabelle Théry-Parisot

\section{To cite this version:}

Pierre Bodu, Hélène Salomon, Jessica Lacarrière, Michael Baillet, Michèle Ballinger, et al.. Un gisement châtelperronien de plein air dans le Bassin parisien : les Bossats à Ormesson (Seine-etMarne). Gallia Préhistoire - Préhistoire de la France dans son contexte européen, 2017, 57, pp.3-64. 10.4000/galliap.478 . hal-02009531

\section{HAL Id: hal-02009531 \\ https://hal.science/hal-02009531}

Submitted on 18 Dec 2019

HAL is a multi-disciplinary open access archive for the deposit and dissemination of scientific research documents, whether they are published or not. The documents may come from teaching and research institutions in France or abroad, or from public or private research centers.
L'archive ouverte pluridisciplinaire HAL, est destinée au dépôt et à la diffusion de documents scientifiques de niveau recherche, publiés ou non, émanant des établissements d'enseignement et de recherche français ou étrangers, des laboratoires publics ou privés.

\section{(ㅇ)(1) $\$$}

Distributed under a Creative Commons Attribution - NonCommercial - NoDerivatives $\mid 4.0$ 


\title{
Un gisement châtelperronien de plein air dans le Bassin parisien : les Bossats à Ormesson (Seine-et-Marne)
}

\author{
Pierre Bodu*, Hélène Salomon**, Jesica Lacarrière***, Michael Balllet****, Michèle Ballinger*, \\ Henrl-Georges Naton*****, ISABelle ThérY-PARISOT ${ }^{* * * * * *}$
}

Reçu le 27 janvier 2017 - Accepté après révision le 10 avril 2017 - Modifié le 16 mai 2017

Mots ctés. Ormesson, Île-de-France, Châtelperronien, vaste occupation de plein air, couteau de Châtelperron, production lamellaire, technologie lithique, tracéologie, pigments

Résumé. Le gisement les Bossats à Ormesson, outre ses niveaux badegoulien, solutréen, gravettien et moustérien, a également livré une occupation châtelperronienne sur une grande surface, occupation pelliculaire et dont l'intégrité n'a pas souffert de mélanges ou de façon très limitée avec des niveaux plus anciens ou plus récents. Fouillé au sein de deux sondages, les sondages 3 et 50 ouverts respectivement sur 16 et $2 \mathrm{~m}^{2}$, mais d'une surface estimée à $150 \mathrm{~m}^{2}$ minimum, ce niveau livre déjà un certain nombre d'informations concernant l'industrie lithique, mais également les matières colorantes dont on connaît, à Ormesson, d'autres indices dans le niveau moustérien ainsi que les ressources animales et végétales. Si dans les années qui viennent, les efforts sont portés vers l'exploitation des niveaux plus récents, nous envisageons une fouille extensive du Châtelperronien dans 4 à 5 ans. Sans attendre ce délai, cet article se veut, avant tout, une première présentation de ce nouveau gisement châtelperronien, le plus septentrional à I'heure actuelle. Mais il pose également un certain nombre de jalons concernant les caractères de l'industrie lithique,
Kerwords. Ormesson, Île-de-France, Châtelperronian, large open-air site, Châtelperronian knife, bladelet production, lithic technology, traceology, pigments

Aвstract. Les Bossats site at Ormesson, in addition to its Badegoulian, Solutrean, Gravettian and Mousterian levels, has also delivered a Châtelperronian occupation over a large area, in the form of a thin layer and whose integrity has not suffered from mixtures, or to a very limited extent, with older or newer layers. This occupation has been excavated in two test-pits, test-pits 3 and 50 of respectively 16 and 2 square meters, but with an estimated surface area of 150 square meters. This level already delivers a certain amount of information concerning the lithic industry but also the colouring materials, already present at Ormesson in the Mousterian level, as well as on animal and vegetable resources. If in the years to come efforts are directed towards the exploitation of the younger levels, we plan an extensive excavation of the Châtelperronian in 4 to 5 years. In the meantime, this article is intended as a first presentation of this new Châtelperronian occupation, the northernmost one known at the present time. But it also lays down a certain number of milestones concerning the characteristics of the lithic industry, especially the

* UMR 7041 ArScAn - Maison René-Ginouvès, 21 allée de l’Université, 92023 Nanterre cedex, pierre.bodu@mae.u-paris10.fr | michele. ballinger@mae.u-paris10.fr

** Service de Préhistoire - Université de Liège (ULg), Place du XX Août,7, 4000 Liège, Belgique, helene.salomon@ulg.ac.be

*** UMR 5608 TRACES - CNRS, Université de Toulouse Jean-Jaurès, maison de la recherche, 5 allées A.-Machado, 31058 Toulouse cedex 9, jessic.laca@gmail.com

**** Université Bordeaux, CNRS, ministère de la Culture et de la Communication - PACEA, Bât. B8, Allée Geoffroy Saint-Hilaire, CS 50023, 33600 Pessac cedex \& Universidad de Cantabria - Dept. Ciencias históricas, av. de los Castros, 39005 Santander, Espagne, michael.baillet@ubordeaux.fr

***** GéoArchéon, 30 rue de la victoire, 55210 Viéville-sous-les-Côtes, géoarcheon@geoarcheon.fr

****** Université Côte d'Azur, CNRS, CEPAM - UMR 7264 - Maison des Sciences de l'Homme et de la Société Sud-Est, 24 avenue des Diables Bleus, 06357 Nice cedex 4, isabelle.thery@cepam.cnrs.fr 
en particulier la production laminaire grâce à la réalisation de nombreux remontages explicites et aussi la présence et le rôle de la production lamellaire. En ce sens, le gisement d'Ormesson participe de façon évidente au débat concernant les productions microlithiques du Châtelperronien et les relations que cette industrie dite de transition a pu entretenir d'une part avec le Moustérien et d'autre part avec le Proto-Aurignacien. Comme leurs prédécesseurs moustériens, mais aussi les groupes qui ont occupé le site bien après eux, les Châtelperroniens d'Ormesson ont sans doute tiré parti d'une topographie particulière de la vallée à cet endroit, favorable au rabattage du gibier. Comme eux, ils ont également su trouver à proximité de l'eau, des ressources végétales et du silex. Comme eux enfin, leur occupation a bénéficié d'un recouvrement rapide par des sédiments, sableux en l'occurrence, qui autorise une analyse palethnographique relativement inédite pour cette période. La petite taille de la surface actuellement fouillée est certes une limitation à l'interprétation économique et fonctionnelle globale de ce niveau, mais elle n'empêche pas un questionnement assez poussé sur les différentes catégories d'outils représentées (couteaux, burins) ou absentes (grattoirs) en l'état actuel de la fouille. Les premiers résultats de l'analyse de la faune et des charbons de bois sont présentés, enrichissant cette documentation, sur la sphère technique, de données plus contextuelles.

Malgré un certain isolement géographique, les Bossats, n'en est pas moins partie prenante d'un territoire châtelperronien «septentrional » qui comporte la célèbre grotte du Renne à Arcy-sur-Cure ou encore celle de la Roche-au-Loup à Merry-sur-Yonne (Yonne). Quelques premières comparaisons sont esquissées avec ces deux sites. Une discussion est amorcée à propos de cette pauvreté apparente en gisements châtelperroniens au nord de la Loire qui est peut-être plus à mettre en relation avec l'état de la recherche qu'elle ne serait liée à une véritable faiblesse de l'occupation. blade production, through the realization of numerous explicit refits and also the presence and role of bladelet production. In this sense, the Ormesson site is an obvious part of the debate concerning the microlithic productions of the Châtelperronian and the relations which this so-called transitional industry was able to maintain with the Mousterian, as well as with the ProtoAurignacian.

Like their Mousterian predecessors, but also like the groups that occupied the site after them, the Châtelperronians from Ormesson undoubtedly benefited from a particular topography of the valley at this point, favourable to the driving of game. Like them, they were also able to find water, vegetal resources and flint nearby. Like them, their occupation was rapidly covered by sediments, sandy in this case, which allows a relatively new palethnographic analysis for this period. The small size of the surface presently excavated is certainly a limitation to the overall economic and functional interpretation of this level, but it does not prevent a fairly detailed questioning of the various categories of tools represented (knives, burins) or absent (end-scrapers) in the current state of the excavation. The first results of the faunal and charcoal analysis are presented, enriching this documentation, on the technical side, with more contextual data.

In spite of a certain geographical isolation, les Bossats are nonetheless part of a 'northern' Châtelperronian territory which includes the famous Renne at Arcy-sur-Cure or Roche-au-Loup at Merry-sur-Yonne (Yonne) caves. Some initial comparisons are outlined with these two sites. A discussion is also initiated about this apparent scarcity in Châtelperronian sites north of the Loire River, which is perhaps more related to the actual state of research rather than to a real lack of occupations. 
Le Châtelperronien est probablement l'une des périodes du Paléolithique supérieur qui suscite le plus de débats et donc de polémiques concernant ses auteurs, même si les restes humains associés à cette industrie lithique et osseuse sont, pour l'instant, ceux de néandertaliens (Leroi-Gourhan, 1958, 1964 ; Lévêque et Vandermeersch, 1980 ; Vandermeersch, 1984 ; Lévêque et al., 1993 ; Hublin et al., 1996 ; Zollikofer et al., 2002) $)^{1}$. Il n'en reste pas moins que le nombre limité de vestiges anthropologiques trouvés dans de rares sites châtelperroniens (Saint-Césaire, Arcy-sur-Cure), les contextes et les conditions parfois délicates de leur découverte et les caractéristiques très «Paléolithique supérieur» de l'industrie lithique, mais aussi et surtout de l'industrie et de la parure en matière dure d'origine animale, génèrent des questionnements et des doutes chez un certain nombre de chercheurs et ce faisant des freins à l'affirmation d'une attribution anthropologique définitive. Un article très récemment paru (Welker et al., 2016) fait état de la découverte d'une vingtaine de fragments supplémentaires de vestiges osseux humains dans les niveaux châtelperroniens de la Grotte du Renne à Arcy-sur-Cure, de leur attribution à l'homme de Néandertal et de datations cohérentes avec le Châtelperronien. Il est trivial de dire que Néandertaliens comme Hommes anatomiquement modernes avaient les capacités cognitives et bien évidemment physiques pour réaliser ces industries lithique comme osseuse mais en l'état de la recherche et des débats où des scénarios différents, parfois d'ordre idéologique, s'affrontent, il serait plus qu'utile de découvrir de nouveaux gisements susceptibles de répondre à ces interrogations si centrales dans notre discipline. Quand la fiabilité des fouilles ou l'état de conservation des sites ne sont pas remis en question, ce sont les résultats des datations qui engendrent de vives discussions, ou encore des interprétations multiples sur la maîtrise du travail des matières osseuses ou des parures par les néandertaliens qui suscitent des polémiques exacerbées (Caron et al., 2011 ; d'Errico et al., 1998). Pourtant loin des débats parfois houleux qui concernent les interprétations de l'art préhistorique puisque les châtelperroniens n'en réalisaient pas encore beaucoup en dehors des parures, ce moment dit de «transition» entre deux humanités est cependant un sujet de discorde tout aussi virulent pour un certain nombre de préhistoriens (Bar-Yosef and Bordes, 2010 ; Higham et al., 2010 ; Hublin et al., 2012 ; Mellars, 2010 ; Roussel et al., 2016 ; Taborin, 1990 ; White, 2001). Il convient donc d'essayer de poser de nouvelles bases pour des discussions plus sereines, pour des études scientifiques moins entachées de scénarios anthropologiques «éventuels», car on ne doit pas se satisfaire de ce trop faible nombre de restes néandertaliens provenant de rares gisements, et dont les conditions de découverte sont régulièrement

1. À l'exception d'une dent humaine moderne découverte aux Roches d'Abilly (Indre-et-Loire) dans un niveau châtelperronien dont le contexte est également l'objet de discussions (Aubry et al., 2012; Aubry et al., 2013). discutées. Il n'est pas intéressant de forcer la documentation archéologique dont nous disposons actuellement. Il est plutôt nécessaire de découvrir des gisements châtelperroniens intègres et de rediscuter des séquences stratigraphiques plus ou moins anciennes comme le font par exemple M. Soressi et M. Roussel aux Cottés (Roussel and Soressi, 2013) ou encore J.-G. Bordes au Piage (Bordes, 2002; Zilhão et al., 2006).

Le gisement les Bossats à Ormesson (Seine-et-Marne) fait désormais partie des ensembles châtelperroniens intègres (fig. 1). Il est déjà connu pour ses occupations solutréenne (Bodu et al., 2014a), gravettienne (Bodu et al., 2011 ; Lacarrière et al., 2015), mais aussi pour son niveau attribué à un Moustérien de débitage discoïde (Bodu et al., 2014 b; Mathis et al., 2014) et dans une bien moindre mesure pour un petit ensemble lithique attribué au Badegoulien (fig. 2). La récurrence des occupations paléolithiques s'explique aisément par la topographie des lieux (promontoire, vallée se rétrécissant), par les protections offertes par l'environnement gréseux, mais aussi par la proximité de différents cours d'eau dont le Loing et par l'abondance de bons silex à moins de $5 \mathrm{~km}$ (fig. 3). Le Solutréen se marque par l'existence de plusieurs structures d'habitat composées de nombreux gros blocs de concrétions de calcite (Bodu et al., 2014a), le Gravettien offre un locus aux activités multiples et où l'organisation spatiale est admirablement bien structurée autour d'aires de combustion (Bodu et al., 2011; Lacarrière et al., 2015), le niveau moustérien qui s'étend à minima sur $800 \mathrm{~m}^{2}$ a livré, entre autres, un nombre très important de matières colorantes parmi lesquelles certaines portent des traces de raclage et/ou d'abrasion révélatrices de la production de poudre rouge riche en hématite (Bodu et al., 2014 b; Mathis et al., 2014). En 2014, un

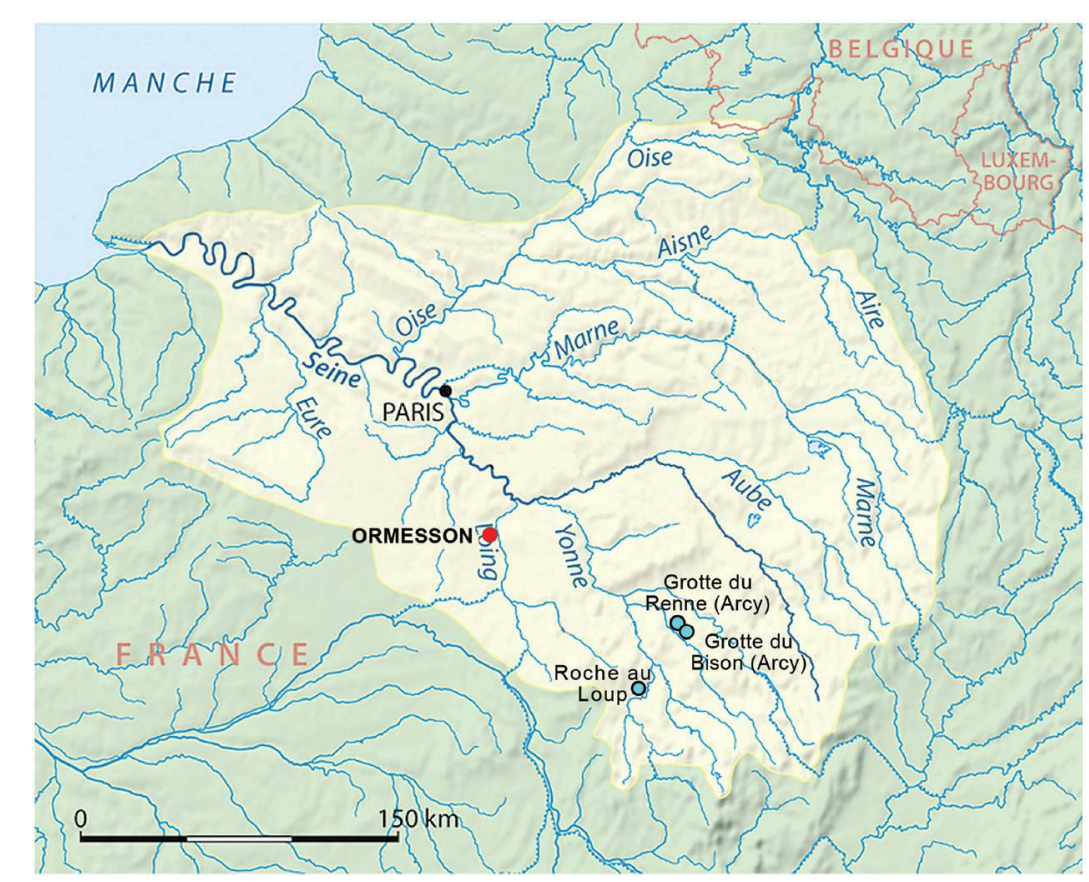

Fig. 1 - Localisation du gisement les Bossats, Ormesson, Seine-etMarne (DAO : M. Ballinger, CNRS).

Location of les Bossats, Ormesson, Seine-et-Marne (CAD: M. Ballinger, CNRS). 


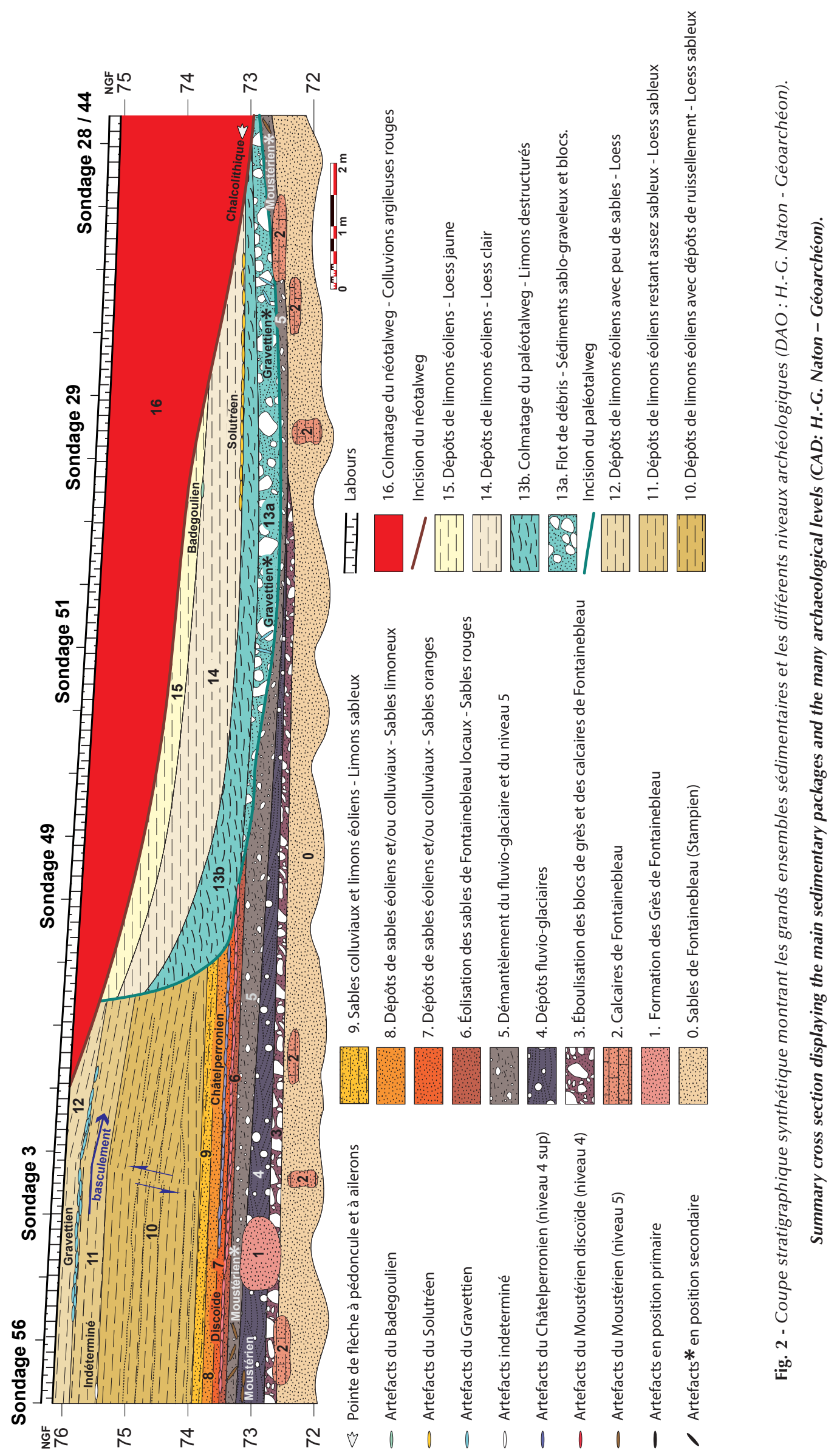



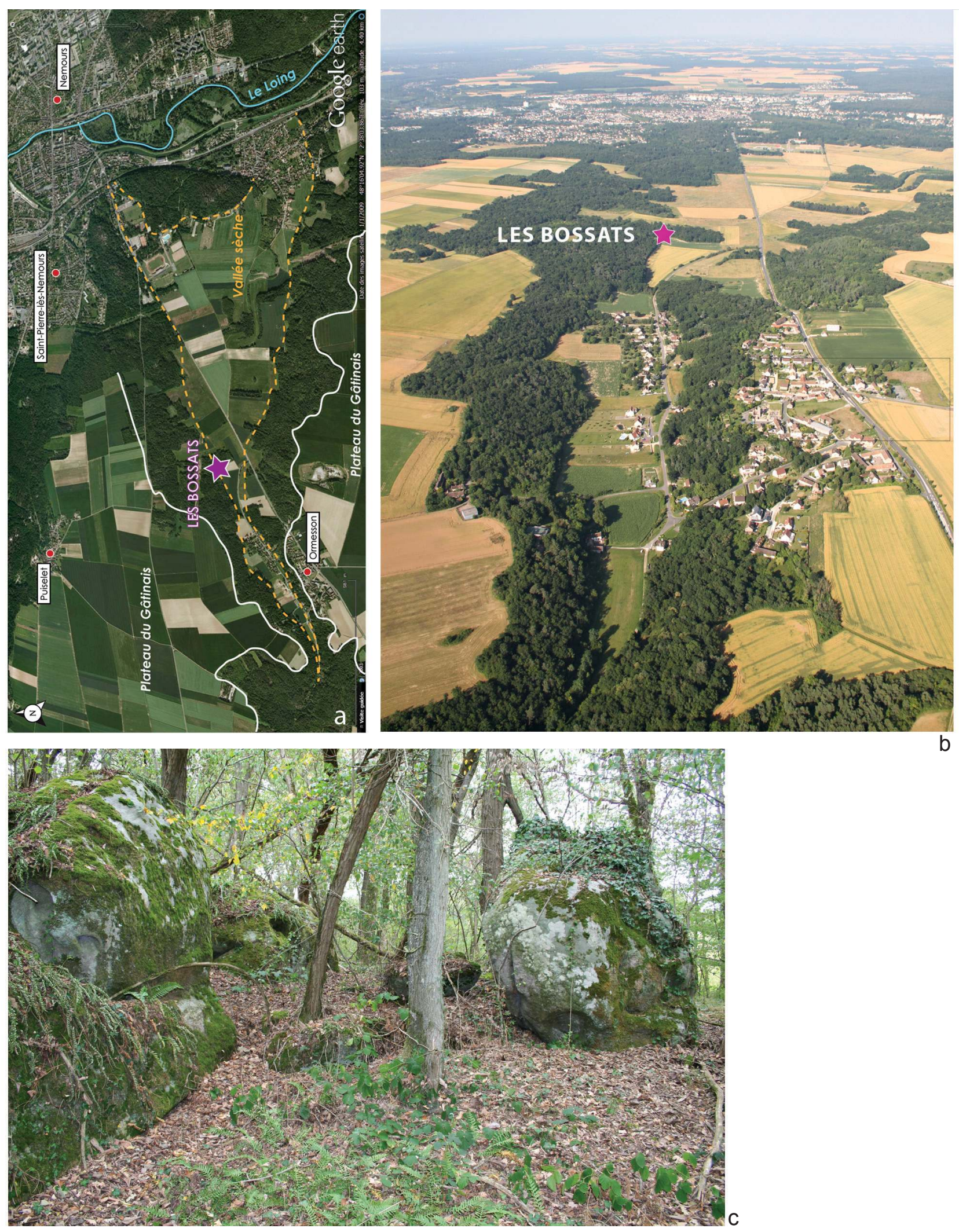

Fig. 3 - a : Photographie aérienne de la vallée menant de Nemours à Ormesson et localisation du site les Bossats (étoile ; (c) : E. Fortier) ; b : Localisation du gisement les Bossats au rétrécissement de la vallée, des affleurements de silex campanien et de la vallée du Loing, sur photo aérienne (Google Earth) ; c : Blocs de grès situés en bordure du gisement les Bossats (@): P. Bodu, CNRS).

a: Aerial photography of the valley leading from Nemours to Ormesson and location of the site les Bossats (star; (: E. Fortier); b: Location of the archaeological site of les Bossats in the narrowing of the valley, of the Campanian flint outcrops and of the Loing Valley, on an aerial view (Google Earth); c: Sandstone blocks at the edge of the site les Bossats (@: P. Bodu, CNRS). 
autre niveau, le quatrième, est venu enrichir la palette des occupations paléolithiques d'Ormesson. Bien que très limité spatialement, ce niveau peut être rattaché, en l'attente d'une confirmation ultérieure, au Badegoulien particulièrement bien représenté dans les gisements proches du bois des Beauregards (commune de Nemours ; Bodu et al., 2007; Schmider, 1971; Schmider et Roblin-Jouve, 2008). Plus récemment en 2015, nous avons eu la confirmation de l'existence d'un cinquième niveau avec deux dates obtenues sur des échantillons organiques provenant d'un foyer isolé (E-F/22, Sondage 50) dans les loess, sans aucun vestige archéologique à proximité. Ces deux dates faites sur os brûlés et terre charbonneuse respectivement de $30110 \pm 390 \mathrm{BP}$ (SacA-43205) et $27930 \pm 300$ BP (SacA-43206), corroborent la position stratigraphique de cette «structure» située à quelques dizaines de cm sous le niveau gravettien, mais leur écart significatif ne permet pas de trancher entre le passage sur le site d'un groupe du Gravettien ancien ou de l'Aurignacien récent qui n'aurait laissé, pour ce que nous en avons documenté pour le moment, que cette aire de combustion. Le tableau ici rapidement dressé est déjà bien suffisant pour faire de ce site un gisement exceptionnel. Or, en 2010, quelques indices discrets nous permettaient d'envisager l'existence d'un sixième ensemble paléolithique inclus dans un niveau sableux à moins d'une vingtaine de $\mathrm{cm}$ au-dessus du Moustérien (Bodu et al., 2014b). Au gré des campagnes de fouille suivantes et notamment en 2013 et 2014, il nous a été possible d'identifier ce niveau comme appartenant au Châtelperronien en en fouillant une surface continue de $16 \mathrm{~m}^{2}$ (sondage 3 ; fig. 4). Cette fouille «extensive» s'est accompagnée

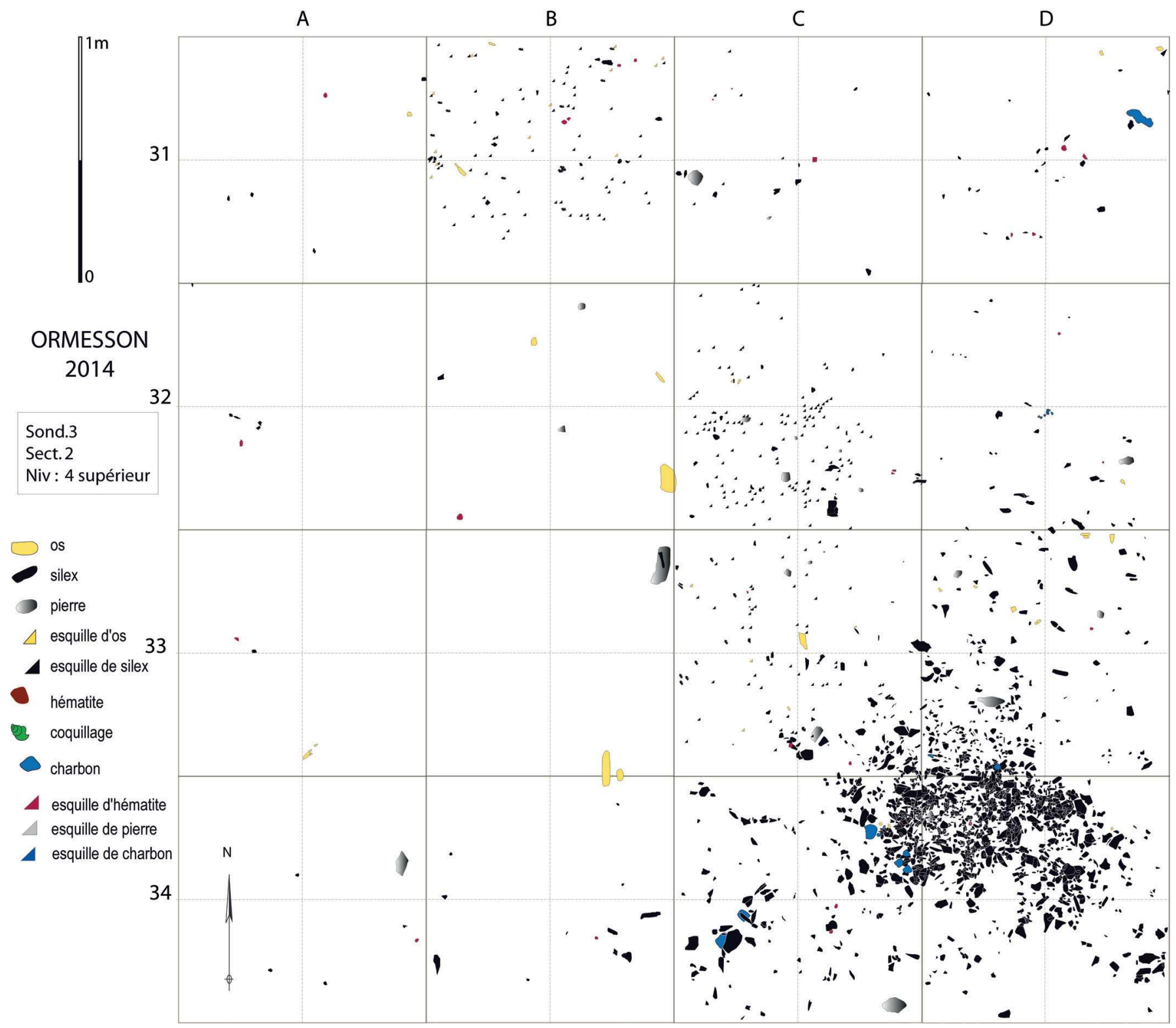

Fig. 4 - Plan du niveau châtelperronien du sondage 3 (DAO : M. Ballinger, CNRS).

Châtelperronian level in test-pit 3 (CAD: M. Ballinger, CNRS). 
de l'identification d'un autre «locus» châtelperronien à une quinzaine de mètres du sondage de la découverte, locus situé à une profondeur comparable et dans un contexte stratigraphique identique (sondage 50 ; fig. 5). Ainsi, le site les Bossats à Ormesson abrite le premier gisement châtelperronien de plein air au nord de la Loire et vient utilement compléter une documentation lacunaire sur un plan géographique, et enrichir notre connaissance des aspects techniques et économiques de la taille de la pierre, mais aussi de l'organisation spatiale et du contexte environnemental d'un tel site. Au-delà de ces aspects, la découverte du niveau châtelperronien à Ormesson relance le débat d'une part sur l'extension géographique présumée de cette industrie dite de transition, sur la pertinence de certains de ses aspects dits «transitionnels» et d'autre part sur les moyens dont disposent les préhistoriens pour distinguer cette industrie somme toute fortement laminaire et parfois lamellaire, des autres panoplies lithiques du Paléolithique supérieur.

\section{UN SITE D'EXCEPTION}

Tous les gisements préhistoriques sont situés à des endroits stratégiques que ce soit pour une topographie particulière, la proximité d'un cours d'eau, la présence de matières premières végétales ou animales ou minérales voire les trois, ou pour des raisons d'ordre plus symbolique ou cultuel. Ormesson ne déroge pas à la règle et plus encore s'affiche très nettement comme un lieu que de nombreuses populations paléolithiques, pour le moins, ont affectionné, et ce pour différentes raisons. La situation topographique des différentes occupations correspond à un promontoire sableux puis loessique dont la pente s'est accentuée au gré des périodes, qui dominait une petite vallée désormais sèche au sud, un paléotalweg à l'est et qui est protégé par des chaos gréseux au nord, à l'ouest et à l'est. Un vaste plateau domine le gisement au nord. Cette situation élevée du site par rapport au fond de vallée et en même temps protégé par des pentes parcourues de blocs de grès et le plateau qui les domine a sans doute constitué un attrait majeur pour les six groupes paléolithiques, au minimum, qui l'ont fréquentée (fig. 6 et 7). Au minimum en effet, car, d'une part, l'absence de toute occupation aurignacienne ${ }^{2}$ pose la question de la survenue de phénomènes de ruissellement, de colluvions qui auraient pu emporter la totalité des niveaux et, d'autre part, les éventuelles occupations postérieures au Solutréo-Badegoulien (Magdalénien, Azilien) dont on connaît de nombreux vestiges dans les gisements proches des Beauregards à Nemours, mais qui manquent ici aux Bossats, ont également pu subir des phénomènes de ruissellements intenses et donc disparaître (Daniel, 1930; Daniel et Daniel, 1953; Delarue et Vignard, 1959; Vignard et Delarue, 1960; Cheynier, 1962; Schmider et Roblin-Jouve, 2008). Un dernier élément enfin constitue sans doute l'un des attraits majeurs du site. C'est sa position stratégique par rapport au passage presque inévitable des troupeaux

2. Même si les premiers articles traitant des occupations badegouliennes du bois des Beauregards attribuaient cette industrie à l'Aurignacien (Delarue et Vignard, 1959). d'herbivores en contrebas, que ces troupeaux soient remontés vers l'ouest sur les vastes plateaux du Gâtinais ou qu'ils soient descendus vers l'est pour atteindre le Loing, empruntant cette voie naturelle que constitue la vallée allant d'Ormesson à Nemours ${ }^{3}$. En amont à l'ouest ainsi qu'à l'aplomb du site, cette dernière est encore étroite ${ }^{4}$ et permet donc des rabattages efficaces de troupeaux. Plus à l'est, la vallée s'élargit et alors un éventuel regroupement des animaux par les hommes est plus difficile à mettre en œuvre (fig. 3 et 6 ). Dans le goulet d'étranglement que constitue cette partie de la vallée d'Ormesson, les chasses aux bisons et autres herbivores ont été vraisemblablement fructueuses comme l'ont été les chasses aux bisons dans le grand ouest américain ou au Canada par les paléoindiens où les troupeaux de bovidés étaient repoussés vers des pièges naturels ou artificiels pour être abattus plus facilement (par ex. le précipice à bisons de Head-Smashed-In en Alberta au Canada; Barsness, 1977). Sans aller jusqu'à écrire que les populations préhistoriques d'Ormesson ont mis à profit les appontements gréseux qui bordent le site à différents endroits pour faire chuter les herbivores, on peut estimer que les chaos rocheux ont constitué, à minima, des promontoires pour observer discrètement ces derniers et peut-être des cachettes idéales pour mieux surprendre les animaux paissant voire des protections lors de charges des bisons énervés.

\section{LE CONTEXTE STRATIGRAPHIQUE}

L'occupation châtelperronienne se situe dans un contexte sédimentaire essentiellement sableux. L'environnement géologique était alors dominé par des blocs de grès stampiens et les sables de Fontainebleau en surface et c'est une végétation sans doute assez rase et peu dense qui devait y pousser. Le sable était donc facilement mobilisable par les agents environnementaux (vent, eau de ruissellement, neige). L'occupation du Châtelperronien est identifiée dans deux sondages, le sondage 3 (fig. 8) et le sondage 50 (fig. 9). Les vestiges de cette occupation se situent en surface de sables de couleur orange, qui recouvrent le niveau moustérien discoïde. Ces sables présentent des taches d'oxydes ferro-manganiques noirs qui indiquent une possible pédogenèse. Ceux qui emballent le Moustérien présentent une coloration nettement plus rouge qui pourrait résulter des activités humaines en relation avec le traitement et l'utilisation de matières colorantes.

Dans la partie nord-ouest du site, le sondage 32 a permis d'observer la présence de niveaux sableux au-dessus d'un bloc de grès de Fontainebleau, qui montrent des caractéristiques similaires aux sables orange observés dans les sondages 3 et 50. Une poche charbonneuse a été découverte dans ces sables (fig. 10) qui a livré la date de $37281 \pm 637 \mathrm{BP}$, cohérente avec une attribution de ce niveau au Châtelperronien.

3. Les chasseurs nous disent qu'à l'heure actuelle c'est une voie empruntée très fréquemment par le gibier.

4. Au point le plus étroit, la vallée délimitée par des versants relativement abrupts encombrés de blocs de grès, présente une largeur de moins de 30 mètres. 


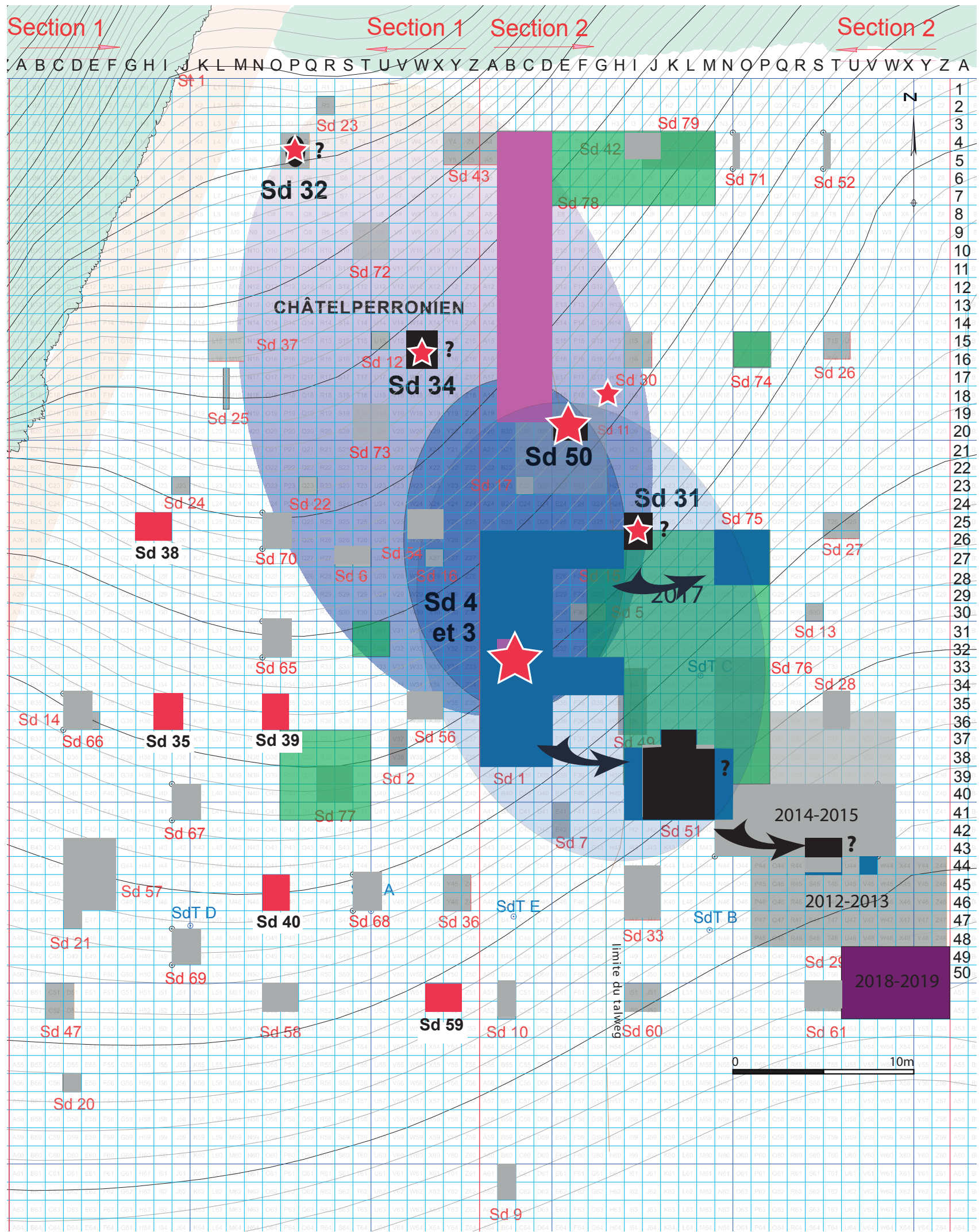

Fig. 5 - Plan de localisation des différents indices châtelperroniens dans le gisement les Bossats (étoiles rouges ; DAO : M. Ballinger, CNRS). 


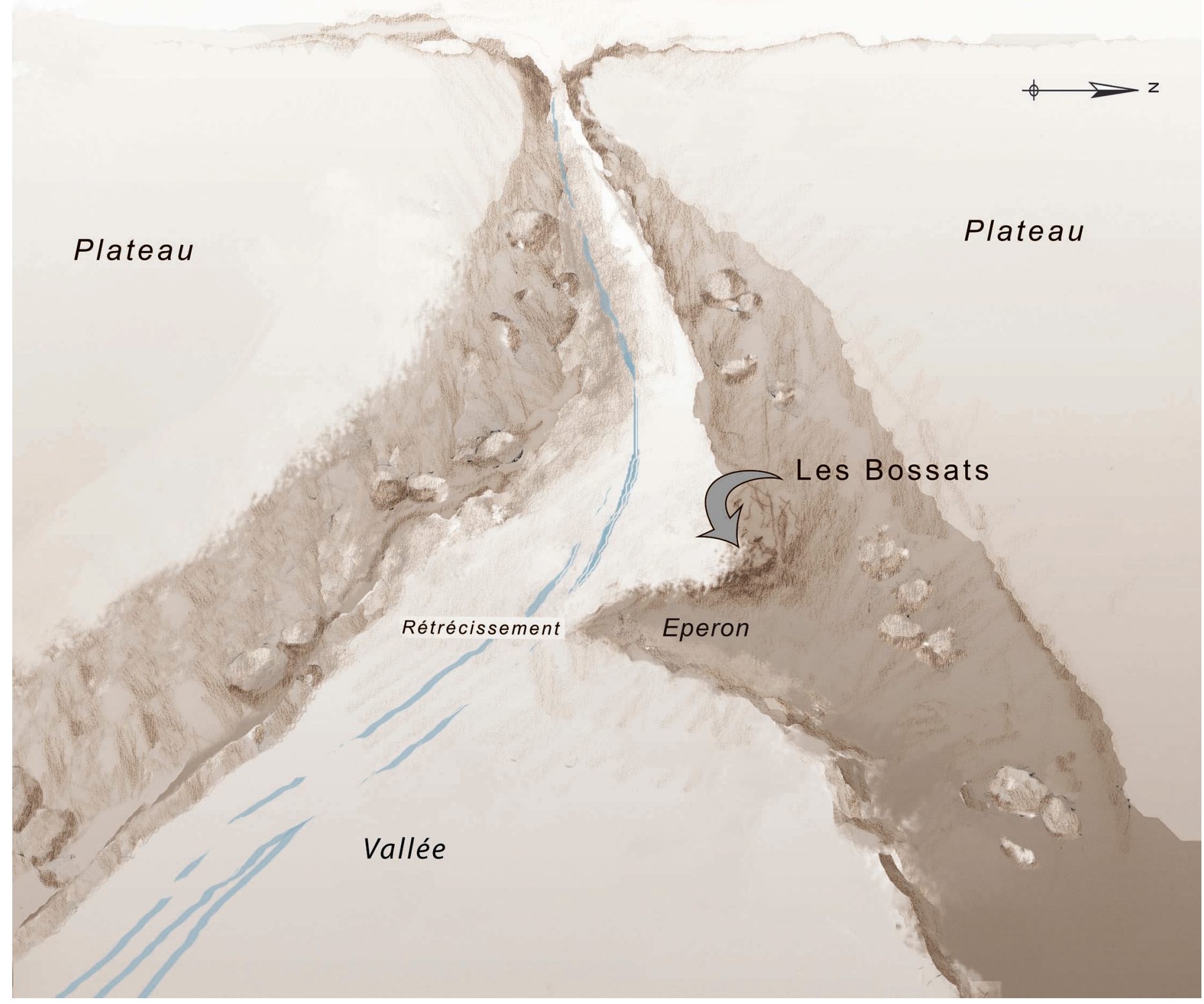

Fig. 6 - Reconstitution de la topographie générale dans et autour du gisement les Bossats (DAO : M. Ballinger, CNRS).

Reconstruction of the landscape topography at les Bossats and in the vicinity (CAD: M. Ballinger, CNRS).

Des limons recouvrent les sables orange qui ennoient l'occupation châtelperronienne. Leur présence s'explique par des apports de longue distance sous la forme de particules balayées et déposées par le vent. La séquence sédimentaire va petit à petit s'enrichir en limons et s'appauvrir en sables. Deux processus sont en jeu : le recouvrement progressif des niveaux moustériens et châtelperroniens par des sables qui va contribuer à leur protection et à une mobilisation très limitée des vestiges et la survenue et l'augmentation plus tardive des apports éoliens de longue distance qui vont, en quelque sorte, fossiliser et donc protéger ces niveaux anciens. Des phénomènes érosifs diachroniques liés à l'écoulement de flux d'eau provenant des plateaux qui dominent le site ont néanmoins affecté l'ensemble châtelperronien, et également le Moustérien. Ils se traduisent par une incision des sédiments postérieurement aux deux occupations et sont à l'origine d'une entité géomorphologique que nous avons dénommée «paléotalweg» au remplissage assez complexe. La partie est du site est plus particulièrement affectée par cette incision d'axe globalement nord-sud ce qui limite l'extension du Châtelperronien dans cette partie orientale du gisement (fig. 11). Cet état de fait est accentué par la formation d'un «néotalweg» qui vient entamer plus récemment le comblement du «paléotalweg» sur sa partie est, en tronquant la séquence jusqu'aux niveaux sédimentaires antérieurs au Moustérien (fig. 12). 


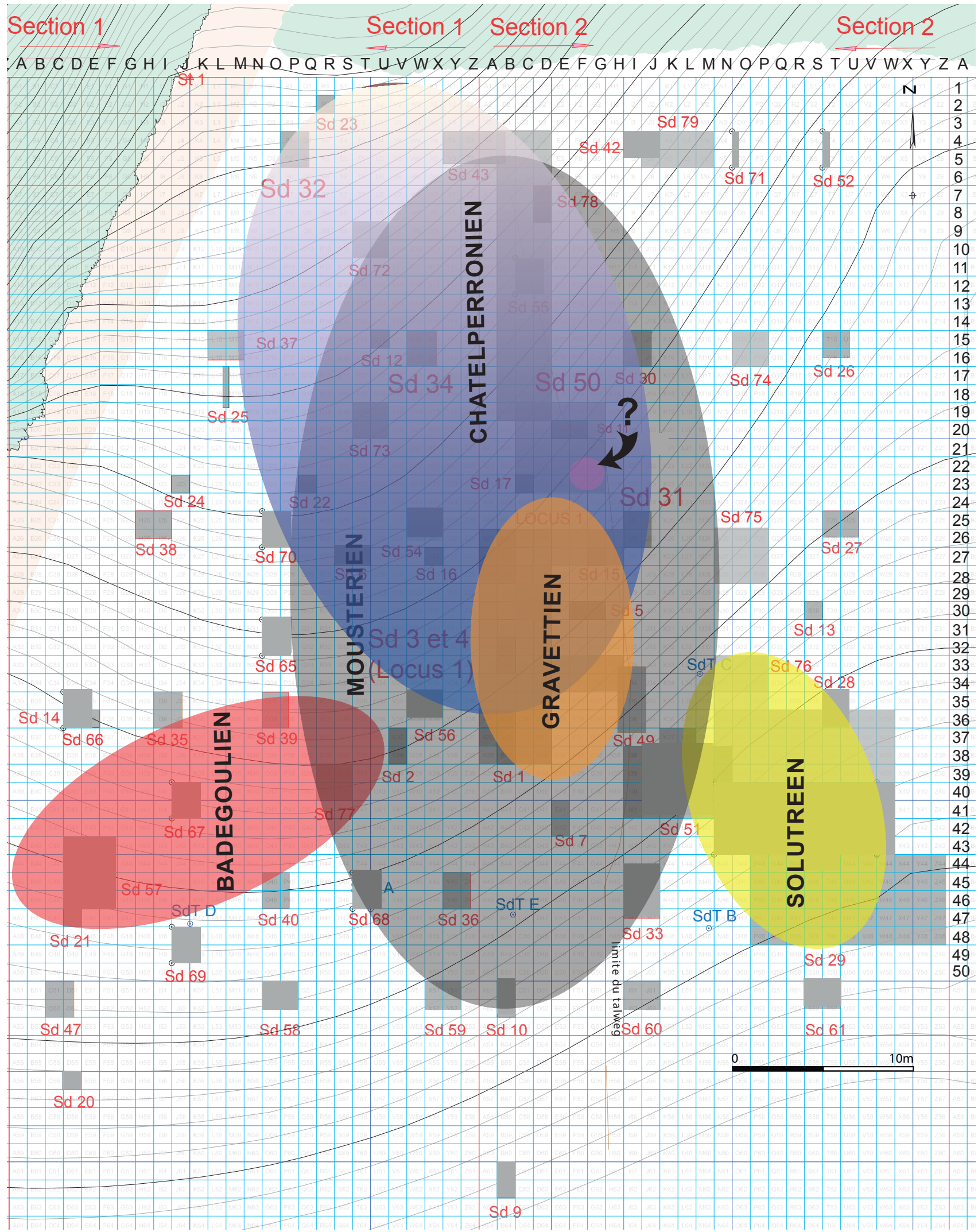

Fig. 7 - Plan de répartition estimée des différents niveaux archéologiques (DAO : M. Ballinger, CNRS). Map of the estimated distribution of the archaeological levels in the different test-pits (CAD: M. Ballinger, CNRS). 


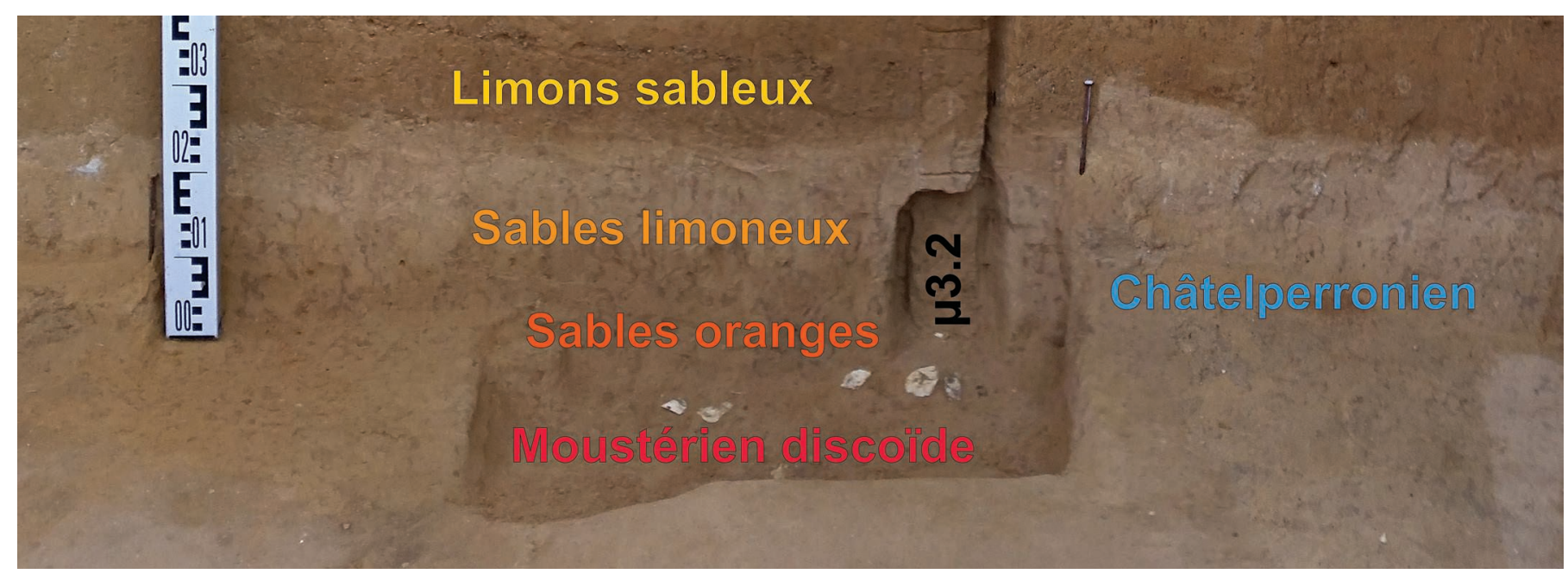

Fig. 8 - Base de la coupe nord du sondage 3 au niveau de la colonne de prélèvements micromorphologiques (ㄷ: H-G. Naton - Géoarchéon). Lower part of the northern profile in test-pit 3, showing the location where the micromorphological samples were taken (৫: H-G. Naton - Géoarchéon).

Fig. 9 - Coupe nord du sondage 50 au-dessus de l'occupation châtelperronienne (): H-G. Naton Géoarchéon).

Northern profile in test-pit 50, above the Châtelperronian occupation level (৫): H-G. Naton Géoarchéon).

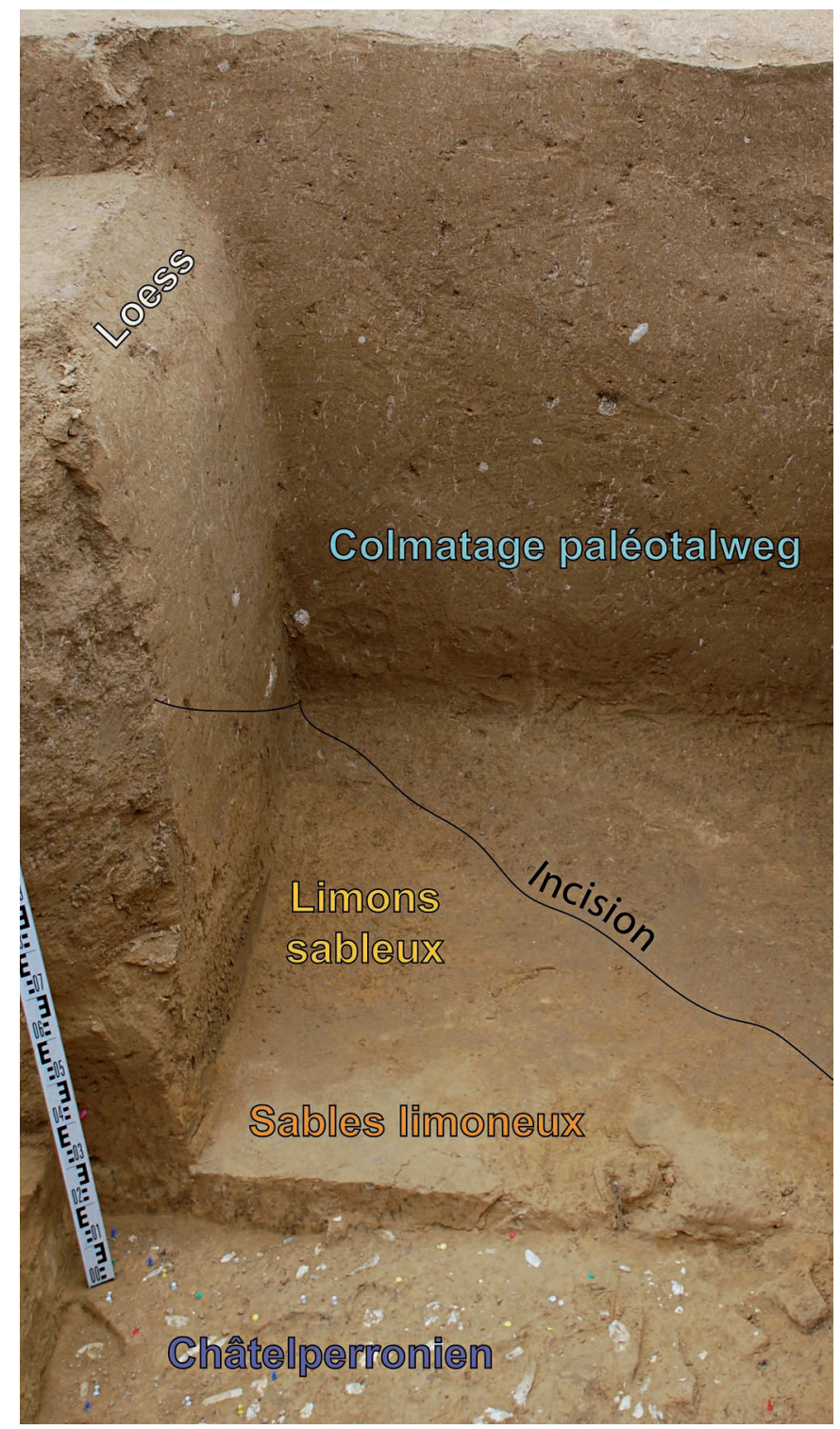




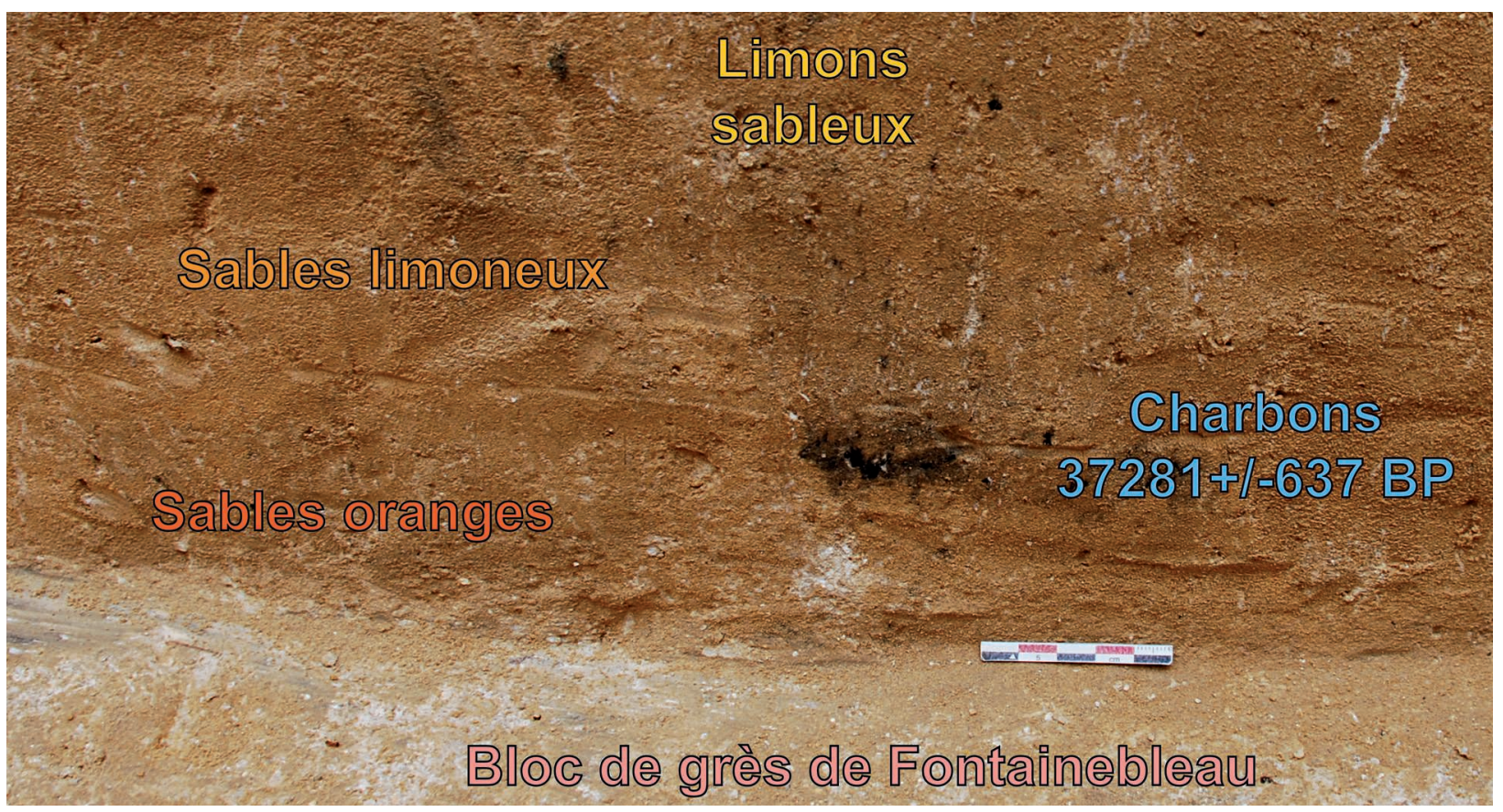

Fig. 10 - Poche charbonneuse au-dessus d'un bloc de grès dans le sondage 32 (๔) : H-G. Naton - Géoarchéon).

Carbon-rich lens above the sandstone block in test-pit 32 (৫): H-G. Naton - Géoarchéon).

\section{Loess}

\section{Faciès colluvial}

\section{Colmatage paléotalweg:}

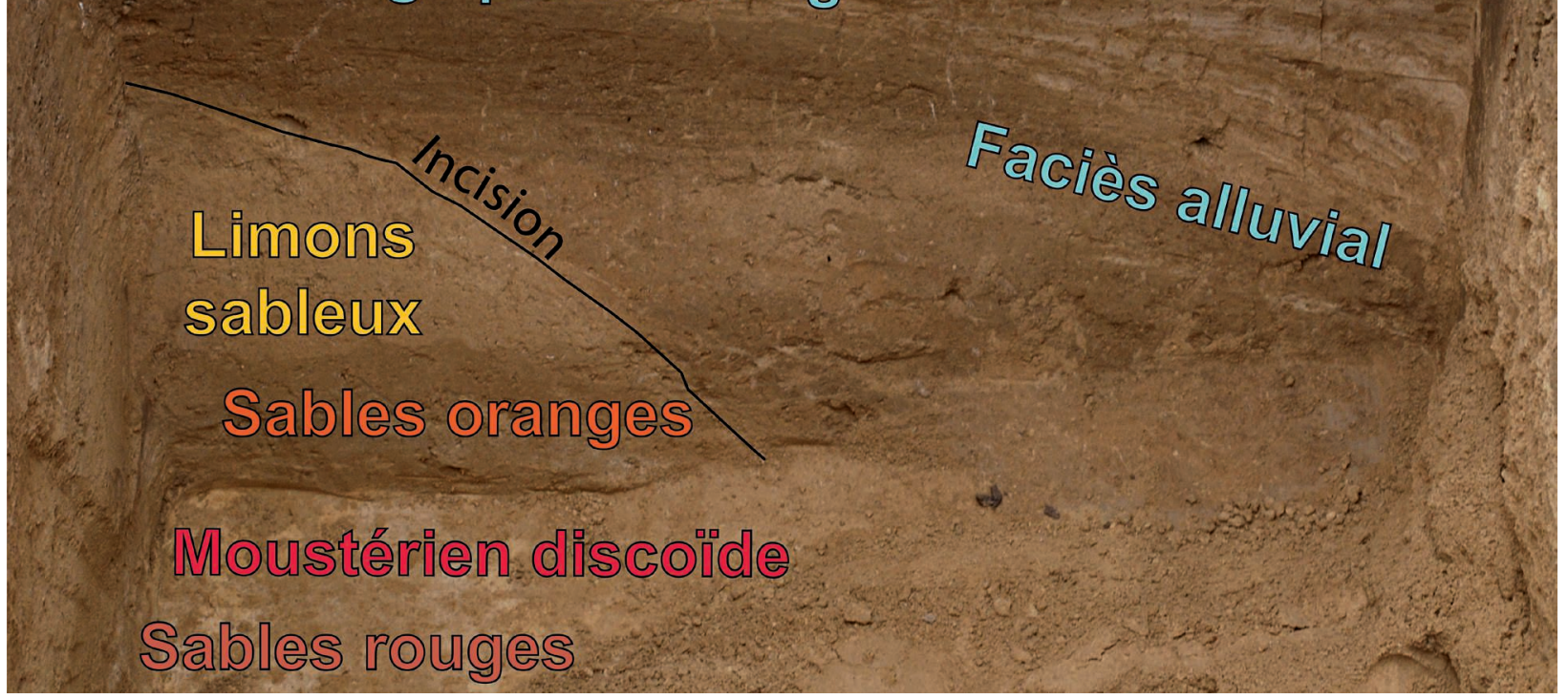

Fig. 11 - Incision et colmatage du paléotalweg dans le sondage 55 (๔ : H-G. Naton - Géoarchéon).

Incision and clogging of the paleothalweg in test-pit 55 (@: H-G. Naton - Géoarchéon). 


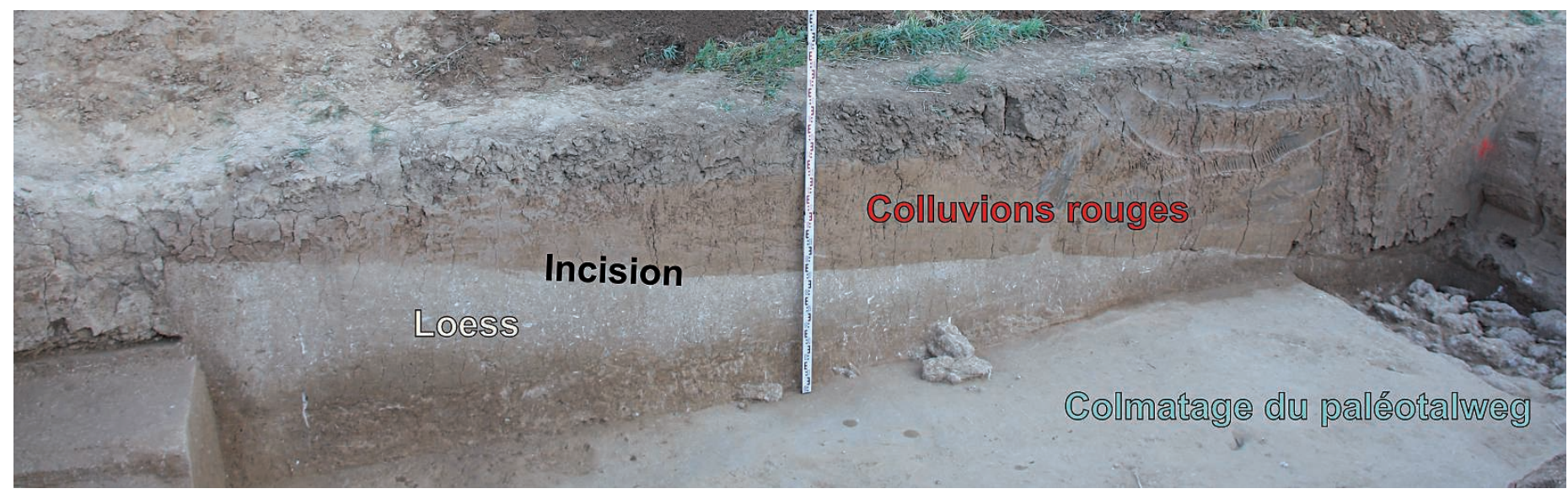

Fig. 12 - Incision et colmatage par les colluvions rouges du néotalweg dans le sondage 29 (৫) : P. Bodu, CNRS et DAO : H-G. Naton - Géoarchéon).

Incision and clogging by red alluvium of the neothalweg in test-pit 29 (৫: P. Bodu, CNRS \& CAD: H-G. Naton - Géoarchéon).

\section{DANS UN GISEMENT MULTISTRATIFIÉ, UNE OCCUPATION CHÂTELPERRONIENNE BIEN CONSERVÉE ET ÉTENDUE}

La partie nord de la parcelle du site les Bossats a fait l'objet, à l'issue de la campagne 2015, de 71 sondages et zones de fouilles d'une surface d'un à une quarantaine de $\mathrm{m}^{2}$ (fig. 13). Les sondages étant distants chacun d'environ 10 mètres, on peut estimer que l'extension des différents niveaux a été bien appréciée. Ainsi sur une surface archéologique potentielle de près de $4000 \mathrm{~m}^{2}$, nous avons sondé et fouillé en sept campagnes environ $400 \mathrm{~m}^{2}$. Le niveau châtelperronien a été rencontré de façon certaine, pour le moment, en deux endroits, dans les sondages 3 et 50 distants de 15 mètres et sur une largeur de près de 10 mètres. Il n'est pas exclu que cette occupation châtelperronienne soit également présente dans d'autres sondages $(31,32,34)$ sous forme plus fugace et avec des éléments moins diagnostiques, ce qui ne nous permet pas d'être affirmatifs (fig. 5). En l'état son extension concernerait donc une surface minimum de près de $150 \mathrm{~m}^{2}$. La présence de couteaux de Châtelperron et d'un débitage laminaire identiques dans les deux sondages accrédite la thèse d'un même ensemble chronoculturel. Mais, bien que les altitudes des pièces trouvées dans les deux sondages soient très proches, nous ne pouvons pas démontrer la stricte contemporanéité des occupations. La jonction physique entre eux, prévue dans 4 à 5 ans une fois les niveaux supérieurs fouillés, permettra d'apporter des réponses définitives à cette interrogation. La petite taille de la surface actuellement fouillée et la distance qui sépare les deux sondages n'empêchent cependant pas d'aborder des domaines importants pour la compréhension de ce technocomplexe si particulier.

C'est au sein du sondage 3 que le niveau châtelperronien, profond d'environ 2,30 $\mathrm{m}$ a été fouillé sur la plus grande surface entre 2012 et 2014, soit $16 \mathrm{~m}^{2}$ contigus (mètres A-D/31 — 34, section 2 ; fig. 4). Ce sont là des limites artificielles, contraintes par la présence, au sommet de la stratigraphie, de l'occupation gravettienne. Dans le sondage 50, en raison de la profondeur du niveau châtelperronien (3 mètres environ) celui-ci n'a pu être fouillé que sur un peu moins de deux mètres carrés (E-F/20, section 2), et nous n'avons démonté en 2014 que les objets provenant d'un mètre carré et demi pour des raisons de temps (fig. 14). Étant donné la petite taille de la zone fouillée dans le sondage 50, il ne nous a pas été possible de vérifier la présence du niveau moustérien sous l'occupation châtelperronienne à cet endroit. En revanche dans le sondage 3, le Châtelperronien, pelliculaire, est situé entre 15 et $20 \mathrm{~cm}$ au-dessus du niveau moustérien (niveau 4 ; fig. 15). Les dispersions verticales, dues sans doute à des terriers et/ou des racines, semblent limitées vers le haut comme vers le bas, même si on n'exclut pas pour le moment une remontée d'objets moustériens de petite taille vers le Châtelperronien. Ainsi, si elles ne sont pas encore possibles dans le sondage 50, fouillé sur une trop petite surface, les premières analyses spatiale et taphonomique du niveau châtelperronien du sondage 3 montrent que cet horizon archéologique a été bien préservé par des dépôts de sable, eux-mêmes recouverts ensuite par une forte sédimentation limoneuse. L'épaisseur de ces dépôts a assuré une protection optimale des niveaux châtelperronien et moustérien, bien qu'ils se trouvent tous deux dans un sable fin très exposé aux différentes formes d'érosion. Ajoutons à cela le fait que la carbonatation des limons sus-jacents, en se diffusant dans les sables inférieurs a également contribué à en renforcer la texture et a participé ainsi à une meilleure préservation de certains restes fauniques. De même, la répartition au sol des différentes catégories de vestiges châtelperroniens, en particulier le silex, témoigne d'une grande cohérence entre un amas de taille ou de rejet situé en C-D/33-34 dans lequel se trouvent concentrés plus de 1200 éclats et fragments de lames et lamelles et des milliers d'esquilles ${ }^{5}$ et des zones périphériques marquées

\footnotetext{
5. Par esquilles, nous entendons des objets d'une taille inférieure à $1 \mathrm{~cm}$. Recueillies lors de la fouille fine, elles sont relevées et dessinées sur des plans au $1 / 20^{\mathrm{e}}$, comme le reste des artefacts. Une grande partie est également découverte au tamisage, dans des tamis dont la maille la plus large est de $4 \mathrm{~mm}$.
} 


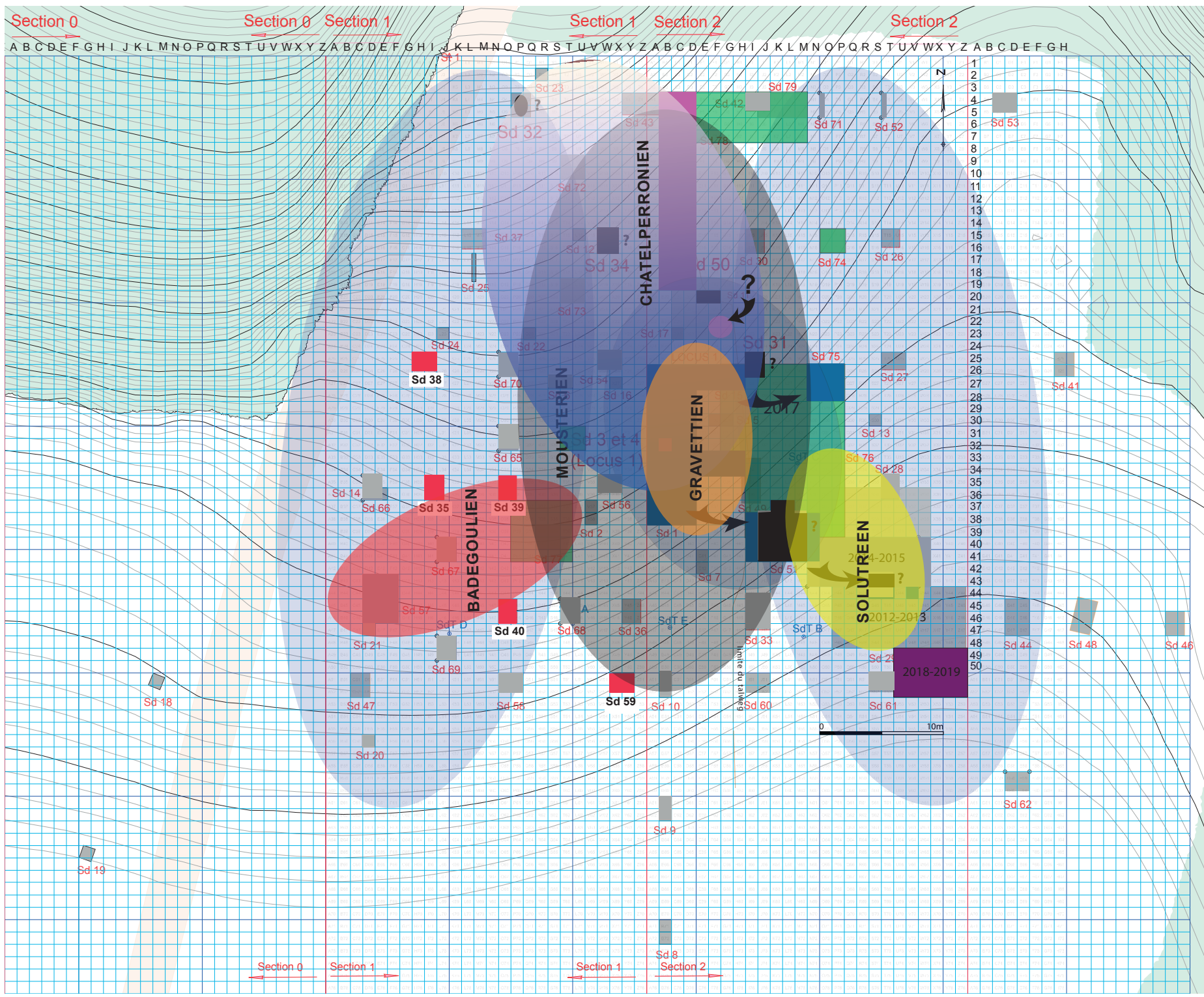

Fig. 13 - Plan de l'ensemble des sondages et aires fouillées entre 2009 et 2015 (DAO : M. Ballinger, CNRS).

Map of the test-pits and areas excavated from 2009 to 2015 (CAD: M. Ballinger, CNRS).

par une moindre densité de vestiges, dont quelques-uns sont néanmoins très évocateurs de certaines activités (couteaux de Châtelperron, burins ; fig. 4, fig. 16). La perception du bon état de conservation du niveau châtelperronien ne serait pas complète si l'on n'évoquait pas l'importance numérique des esquilles ainsi que le grand nombre de raccords et remontages réalisés sur le matériel lithique. Bien que la série lithique soit incomplète puisqu'elle ne représente que ce qui a été trouvé sur $16 \mathrm{~m}^{2}$, il a été possible de remonter une douzaine d'ensembles débités regroupant entre deux et 121 pièces. Au total 465 pièces ont été remontées et/ou raccordées et elles sont accompagnées d'une centaine d'autres pièces associées par la couleur du silex, du cortex, la présence d'un fossile particulier, ce qui au total représente un peu plus de $36 \%$ du silex taillé hors esquilles. Raccords, remontages, très forte présence d'esquilles et de poussière d'esquilles, bon état de conservation des surfaces et des tranchants des silex, comme en témoigne l'analyse tracéologique, tout cela va dans le sens d'une bonne préservation du niveau châtelperronien. La faune, bien que présente, est néanmoins très discrète et la plupart des éléments retrouvés correspondent à des esquilles d'os brûlées, des fragments de petite taille non brûlés, mais dont la surface est corrodée et plus rarement de gros fragments à l'instar d'un tibia de bison découvert en 2010 à environ un mètre au nord de l'amas de silex précédemment évoqué. La présence de fragments de matières colorantes, par nature fragiles, témoigne tout comme celle d'une nappe cendreuse bordant l'amas de silex au sud, du bon état de conservation de ce niveau (fig. 16). L'analyse anthracologique menée par l'une d'entre nous a permis d'identifier le pin et le genévrier, deux espèces également présentes dans le 


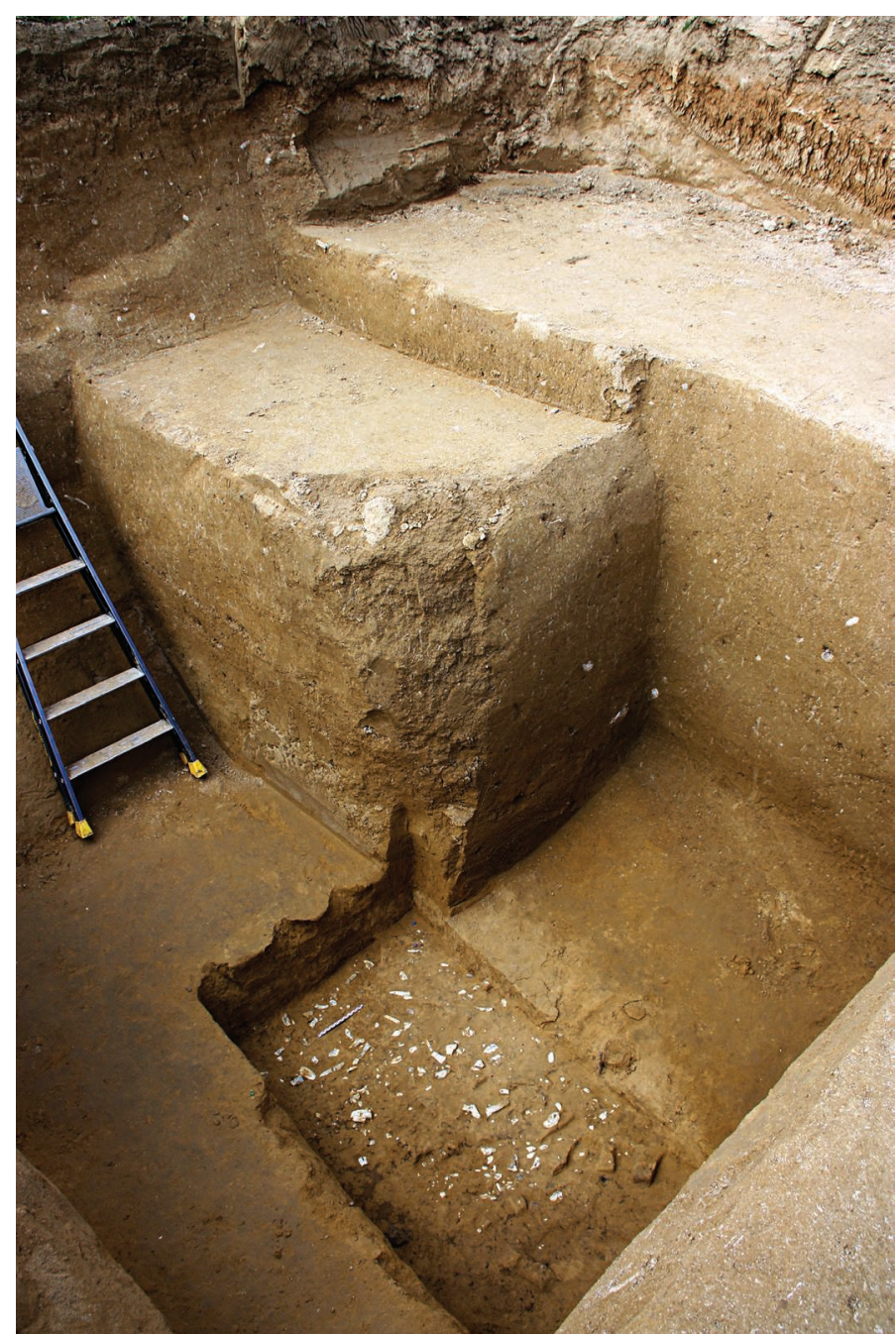

Fig. 14 - Vue du sondage 50 et du niveau châtelperronien au fond (C) : P. Bodu, CNRS).

View of test-pit 50 and the Châtelperronian level at the bottom (C): P. Bodu, CNRS).

niveau moustérien. On ne peut de ce fait écarter l'hypothèse de circulations verticales de charbons de bois du Moustérien vers le Châtelperronien ou l'inverse. Dans le sondage 50, la faible surface fouillée, contrainte par la profondeur du niveau ${ }^{6}$, et sur laquelle les objets ont été recueillis (un mètre carré et demi) n'autorise qu'une observation spatiale réduite. La densité ainsi que la nature des supports lithiques abandonnés (beaucoup de lames et de lamelles et quelques outils, assez peu d'éclats) dans ce mètre carré et le mètre voisin plaident néanmoins en faveur de l'existence d'une aire d'activité plus que d'une zone de rejet (fig. 17). L'identification de près de 24 silex brûlés (hors esquilles) soit $6 \%$ environ de l'ensemble lithique recueilli témoigne de la proximité d'une aire de combustion. La réali-

6. Et donc la nécessité de respecter des paliers de sécurité et de restreindre la surface fouillée en profondeur.

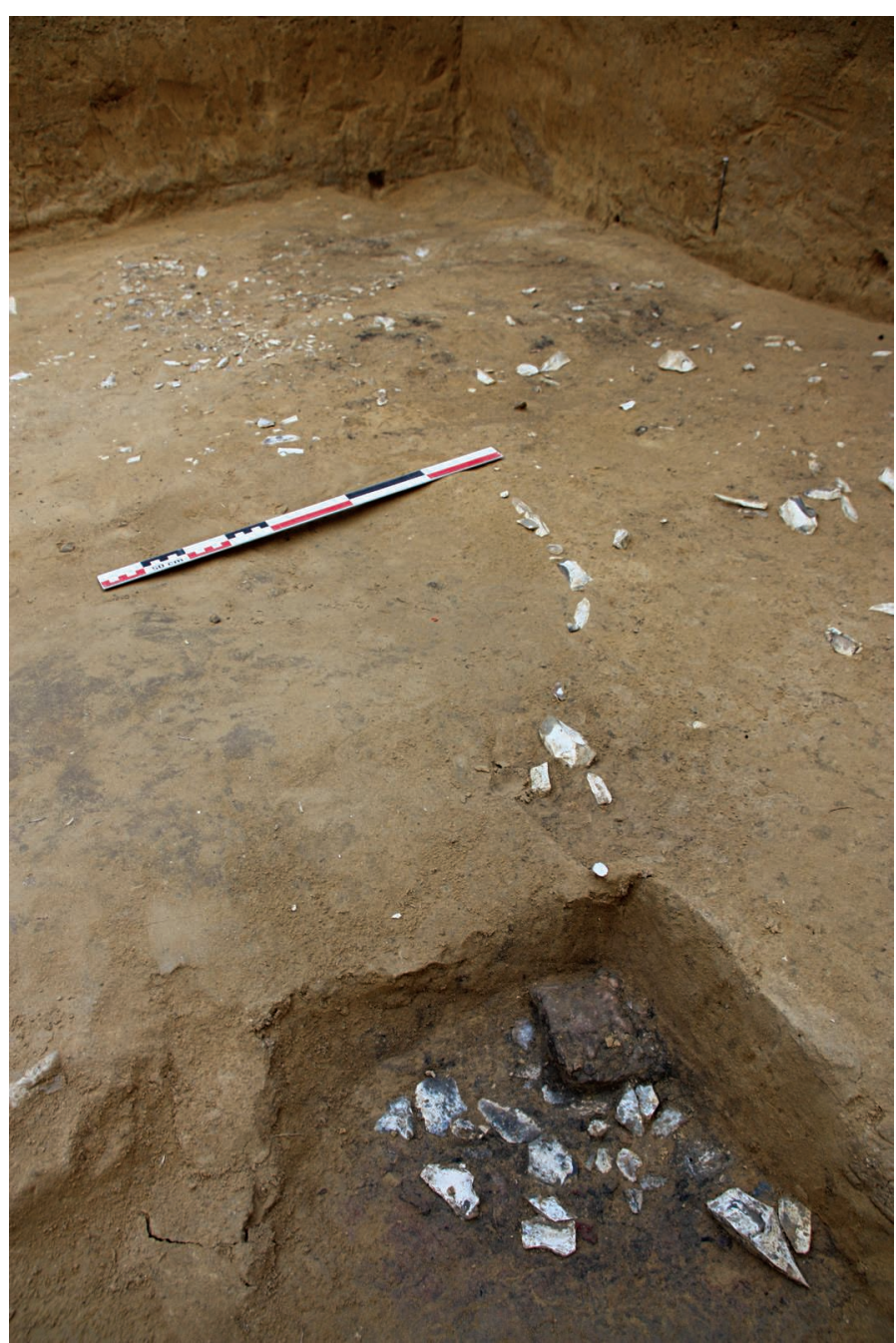

Fig. 15 - Les sols moustérien et châtelperronien superposés dans le sondage 3. Au premier plan, le niveau moustérien, au second plan le niveau châtelperronien (ㄷ: P. Bodu, CNRS).

Superimposed Mousterian and Châtelperronian soils in test-pit 3. In the foreground, the Mousterian level, and at the background, the Châtelperronian level (:: P. Bodu, CNRS).

sation de quelques remontages dont celui d'un débitage relativement complet (nucléus E20.155) démontre le caractère non perturbé et peu dispersé du niveau archéologique. En résumé, le niveau châtelperronien d'Ormesson est bien conservé, plat et pelliculaire, il couvre une surface d'au moins $150 \mathrm{~m}^{2}$, il associe amas de déchets de silex, zone charbonneuse et cendreuse, aires d'activités avec de l'outillage et des supports laminaires et lamellaires. Il est le site châtelperronien le plus septentrional pour le moment, qui plus est de plein air (fig. 18). C'est donc un très bon candidat, comme certains sites de plein air du sud-ouest (Le Basté, Les Tambourets, La Côte ou Canaule II par exemple), pour une étude de type palethnographique sur du Châtelperronien, alors que la Grotte du Renne à Arcy-surCure ou la Roche-à-Pierrot à Saint-Césaire, doivent déjà passer par le filtre d'approches géomorphologique et taphonomique détaillées avant qu'y soit développé ce type d'analyse. C'est 

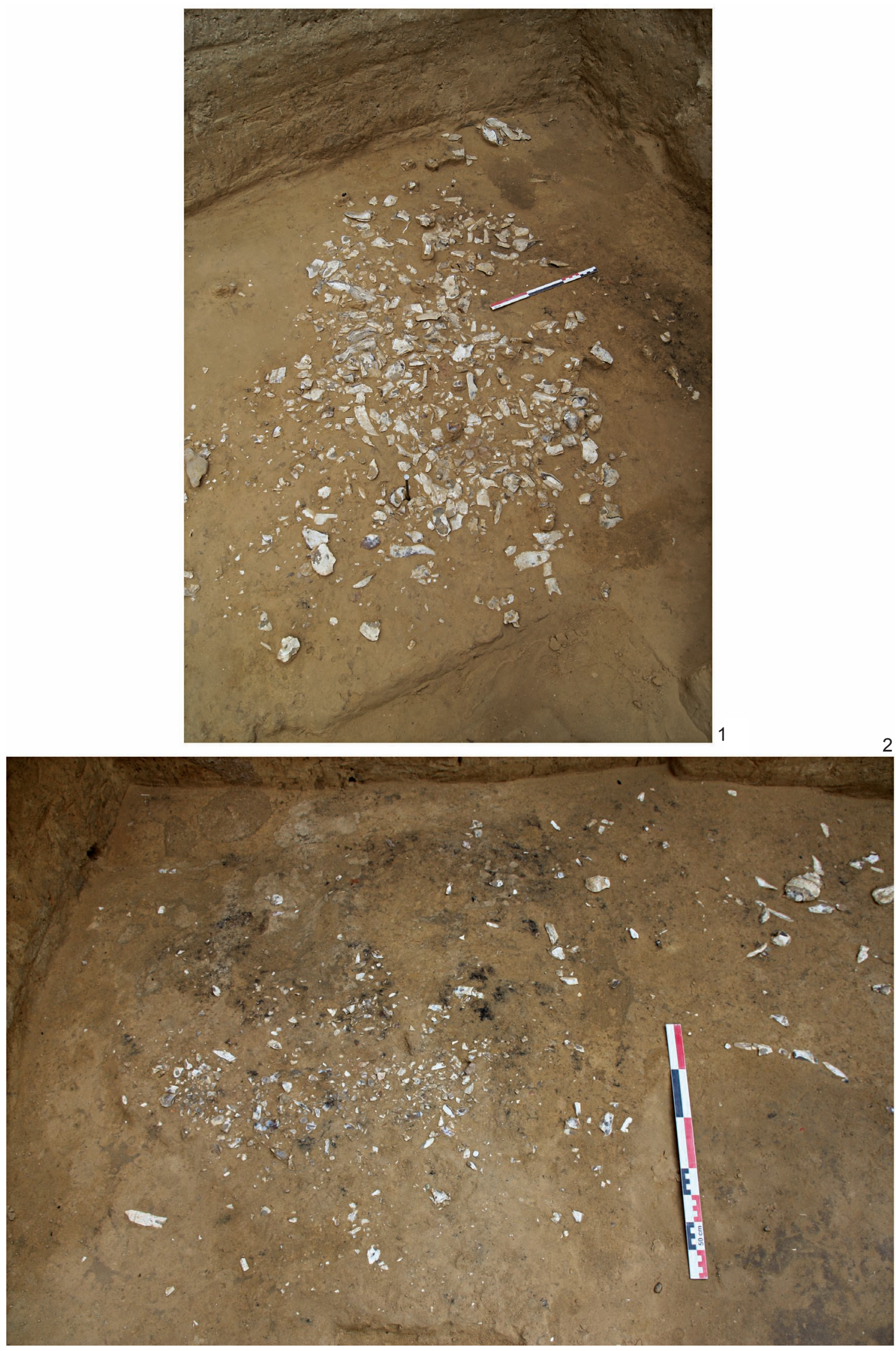

Fig. 16 - Deux vues de l'amas de silex et de la zone cendreuse du niveau châtelperronien du sondage 3 (C): P. Bodu, CNRS).

Two views of the flint concentration and the ash-rich zone within the Châtelperronian level in test-pit 3 (@): P. Bodu, CNRS). 


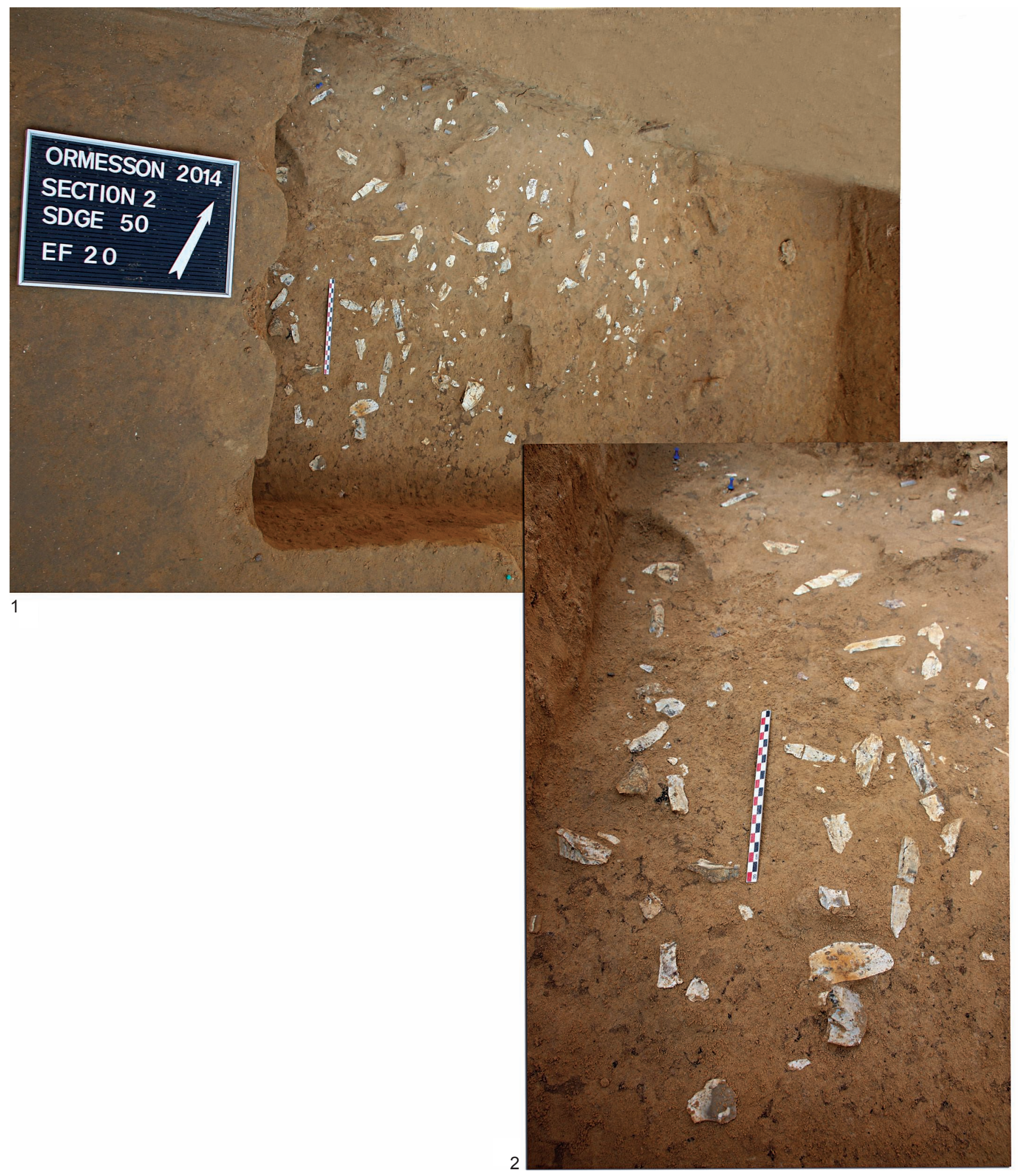

Fig. 17 - L'amas de silex du niveau châtelperronien du sondage 50 : vue générale (1) et vue de détail (2 ; ( : P. Bodu, CNRS). Flint concentration in the Châtelperronian level in test-pit 50: overall view (1) and detail view (2; @: P. Bodu, CNRS). 


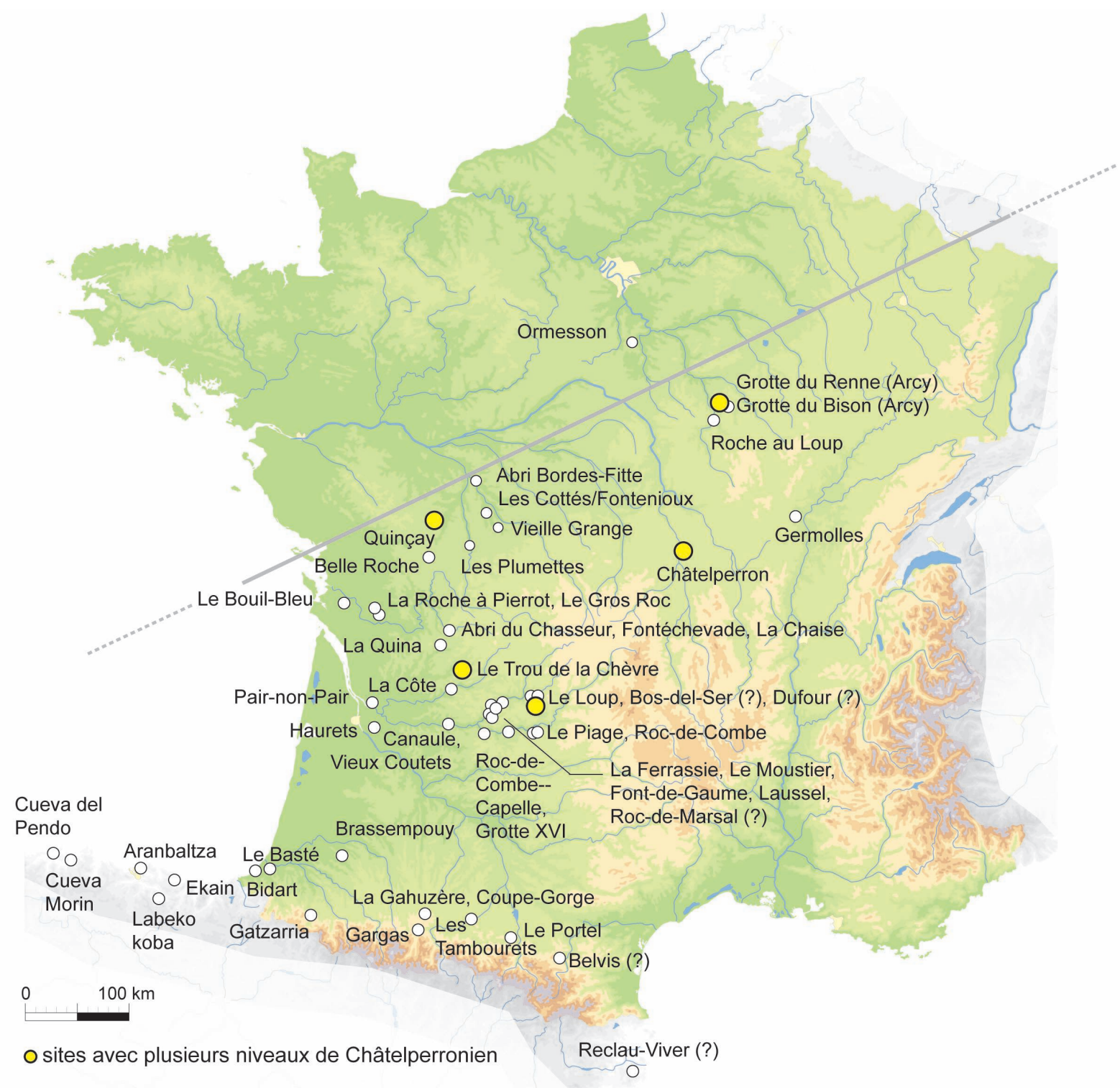

Fig. 18 - Carte de répartitions des gisements châtelperroniens (d'après Roussel et Soressi, 2014).

Distribution map of the Châtelperronian sites (after Roussel and Soressi, 2014).

également un très bon candidat pour une description optimale et sans soupçon de mélange, des mobiliers qu'ils soient d'os ou de silex, ce qui n'est pas toujours le cas des gisements en grotte ou sous abri!

\section{DISCUSSION AUTOUR DES DATES DU NIVEAU CHÂTELPERRONIEN D'ORMESSON}

L'autre intérêt du niveau châtelperronien d'Ormesson est d'être en relation stratigraphique étroite avec l'occupation moustérienne de type discoïde (Bodu et al., 2014b). Dans le sondage 3, ces deux niveaux superposés sont séparés par une épaisseur variable de sédiment sableux de moins d'une vingtaine de centimètres (fig. 15). Le niveau moustérien d'Ormesson a été daté deux fois par thermoluminescence. Les datations effectuées sur deux silex brûlés sont les suivantes $(44,8 \pm 3,4$ ka BP et $49,9 \pm 3,1 \mathrm{ka} \mathrm{BP}$; tabl. I), ce qui correspond à une période finale du Paléolithique moyen au sein du MIS 3 (Bodu et al., 2014 b), en prenant également en compte le fait qu'il s'agit de dates par thermoluminescence qui peuvent donc marquer un certain décalage par rapport aux dates radiocarbone. Ces deux datations sont en total accord avec le caractère discoïde de l'industrie qui se place, dans quelques cavités d'Arcy-sur-Cure notamment (Grotte du Bison, Grotte du Renne, Grotte de l'Hyène), au sommet de la séquence du Paléolithique moyen (Lhomme et al., 2005) précédant donc les occupations châtelperroniennes si riches de la Grotte du Renne. 


\begin{tabular}{|l|l|l|l|}
\hline Origine échantillon & Echantillon daté & Position stratigraphique & Résultats BP non cal \\
\hline Ormesson 2011, 2, Sd 3, B33.272 & Silex brûlé & Niveau 4 & $44.8+/-3.4 \mathrm{ka} \mathrm{BP}$ \\
\hline Ormesson 2011, 3, Sd 3, B33.442 & Silex brûlé & Niveau 4 & $49.9+/-3.1 \mathrm{ka} \mathrm{BP}$ \\
\hline
\end{tabular}

Tabl. I - Tableau des datations TL du niveau moustérien d'Ormesson (Bodu et al., 2013c).

TL dates for the Mousterian level at Ormesson (Bodu et al., 2013c).

\begin{tabular}{|c|c|c|c|c|c|c|}
\hline Origine échantillon & Echantillon daté & Position stratigraphique & Code Laboratoire & Résultats BP non cal & Cal BC 1 sigma & Cal BC 2 sigma \\
\hline Ormesson 2011, Sd 3, B33.350 & OS & Au-dessus niv 4 sup & Erl 16609 & $34450+/-541 \mathrm{BP}$ & 38206-36874 BC & $39026-36442$ BC : $94,4 \%, 36307-36140$ BC : $1 \%$ \\
\hline Ormesson 2011-2012, Sd 3 C34.18-20 & Charbon & vrai niv 4 sup & Erl 17855 & $34005+/-358 \mathrm{BP}$ & $37505-36570 \mathrm{BC}$ & 38134-35804 BC \\
\hline Ormesson 2010, Sd 3, B32.5 & OS & vrai niv 4 sup & Erl 16610 & $29900+/-620 \mathrm{BP}$ & $33070-31723 \mathrm{BC}$ & $34723-33942$ BC : $3,4 \%, 3361731027$ BC : $92 \%$ \\
\hline Ormesson 2014, Sd 3, D33 (15) & Charbon & vrai niv 4 sup & Erl 19456 & $37442+/-643 \mathrm{BP}$ & 40375-39431 BC & $40782-38790 \mathrm{BC}$ \\
\hline Ormesson $2014, \mathrm{Sd} 32$ & Charbon & coupe, sans archéo & Erl 19455 & $37281+/-637$ BP & 40274-39303 BC & 40663-38629 BC \\
\hline Ormesson 2014, Sd 3, C34.316-317 & Charbon & vrai niv 4 sup & Lyon-11864 (Sac A40374) & $37400+/-1100 \mathrm{BP}$ & 1 & 41867-37927 BC \\
\hline Ormesson 2014, Sd 3, C34.316-317 & Charbon & vrai niv 4 sup & Lyon-11660 (Sac A39280) & $39600+/-1400 \mathrm{BP}$ & 1 & $44620-39626 \mathrm{BC}$ \\
\hline
\end{tabular}

Tabl. II - Tableau des datations du niveau châtelperronien d'Ormesson et calibration à partir de la courbe IntCal13 (Reimer et al., 2013).

Radiocarbon dates for the Châtelperronian level at Ormesson. The dates are calibrated using IntCal13 (Reimer et al., 2013).

Les différentes datations obtenues ( 1 sur os, 5 sur charbon de bois ${ }^{7}$ ) sur le niveau châtelperronien d'Ormesson (tabl. II) s'étagent, quant à elles, entre $29900 \pm 620$ BP et $39600 \pm$ $1400 \mathrm{BP}$, soit une tranche chronologique de près de 10000 ans qu'il faut bien évidemment considérer avec beaucoup de prudence eu égard aux écarts types qui vont du simple à plus du double. Quoi qu'il en soit le plus grand nombre de datations semble centré autour de 37000 ans BP, datations sur lesquelles nous reviendrons ultérieurement.

Les datations obtenues sur l'ensemble de la stratigraphie, du niveau 4 moustérien au niveau solutréen, ont été calibrées à partir de la courbe IntCal13 (Reimer et al. 2013) et soumises à une modélisation bayésienne avec le logiciel ChronoModel qui permet un traitement des résultats à plusieurs échelles (version 1.5.0., Lanos et al., 2015). Les résultats sont mis en relation avec la courbe du $\delta^{18} \mathrm{O}$ au Pôle Nord (NGRIP ; Stocker, Johnsen, 2003 ; tabl. III et IV). Nous avons construit ce modèle en réunissant certaines datations au sein d'un même événement lorsque la relation entre les échantillons prélevés est connue (par exemple plusieurs datations sur des vestiges du même type - os, silex - appartenant à une aire de combustion ou à un niveau continu de vestiges archéologiques pour lesquels des raccords et des remontages ont été réalisés). Lorsque la relation n'est pas connue strictement, dans le cas de prélèvements effectués sur des vestiges sans connexion évidente au sein d'un même ensemble chronostratigraphique (qui sont ici les niveaux individualisés à partir de critères archéologiques et sédimentaires), chaque datation représente un événement différent. Les événements sont ensuite réunis au sein de phases chronologiques, c'est-à-dire les niveaux archéologiques et sédimentaires. La déviation standard est deux fois plus élevée pour la datation sur l'os de bison (sd. 3, B32, Erl-16610 : $29900 \pm$ $620 \mathrm{BP})$ que pour les autres datations réalisées sur les vestiges

7. La rareté des restes osseux nous a conduits dans un premier temps à privilégier les datations sur charbons de bois. Un programme complémentaire de datations sur os est prévu dans un court terme.
Tabl. III - Représentation de la modélisation des âges calibrés avant l'année 2000 avec la courbe IntCal13 (Reimer et al., 2013) pour les niveaux gravettien et solutréen. Les résultats sont confrontés à la courbe du North Greenland Ice Core Project (NGRIP) des $\delta^{18} \mathrm{O}$. Les cycles de Dansgaard-Oeschger (DO) se caractérisent par un réchauffement rapide (chiffres noirs au-dessus de la courbe). Trois événements de Heinrich marqués par un refroidissement brutal sont indiqués en bleu (H5 à H3). Sources : ChronoModel (Lanos et al., 2015), NGRIP (Stocker et Johnsen, 2003).

Bayesian model for the radiocarbon dates concerning the Gravettian and Solutrean levels. This model is generated using ChronoModel (Lanos et al., 2015) and IntCal13 (Reimer et al., 2013). The results are presented in calendar dates before year 2000 (K2B) and compared with the (NGRIP) $\delta^{18} \mathrm{O}$ climate record (Stocker and Johnsen, 2003). Dansgaard-Oeschger events (DO) are rapid warming episodes (indicated with black numbers). Three Heinrich events ( $\mathrm{H} 5$ to $\mathrm{H} 3$, indicated in blue) are related to periods of abrupt and rapid cooling.

Tabl. IV - Représentation de la modélisation des âges calibrés avant l'année 2000 (K2B) avec la courbe IntCal13 (Reimer et al., 2013) pour les niveaux moustérien à intermédiaire. Les résultats sont confrontés à la courbe du North Greenland Ice Core Project (NGRIP) des $\delta^{18} \mathrm{O}$. Les cycles de Dansgaard-Oeschger (DO) se caractérisent par un réchauffement rapide (chiffres noirs au-dessus de la courbe). Trois événements de Heinrich marqués par un refroidissement brutal sont indiqués en bleu (H5 à H3). Sources : ChronoModel (Lanos et al., 2015), NGRIP (Stocker et Johnsen, 2003). DAO : H.Salomon.

Bayesian model for the radiocarbon dates concerning the levels from Mousterian to intermediate levels. This model is generated using ChronoModel (Lanos et al., 2015) and IntCal13 (Reimer et al., 2013). The results are presented in calendar dates before year 2000 (K2B) and compared with the (NGRIP) $\delta^{18} \mathrm{O}$ climate record (Stocker and Johnsen, 2003). Dansgaard-Oeschger events (DO) are rapid warming episodes (indicated with black numbers above the curve). Three Heinrich events (H5 to H3, indicated in blue) are related to periods of abrupt and rapid cooling. Sources: ChronoModel (Lanos et al., 2015), NGRIP (Stocker and Johnsen, 2003). DAO: H.Salomon. 

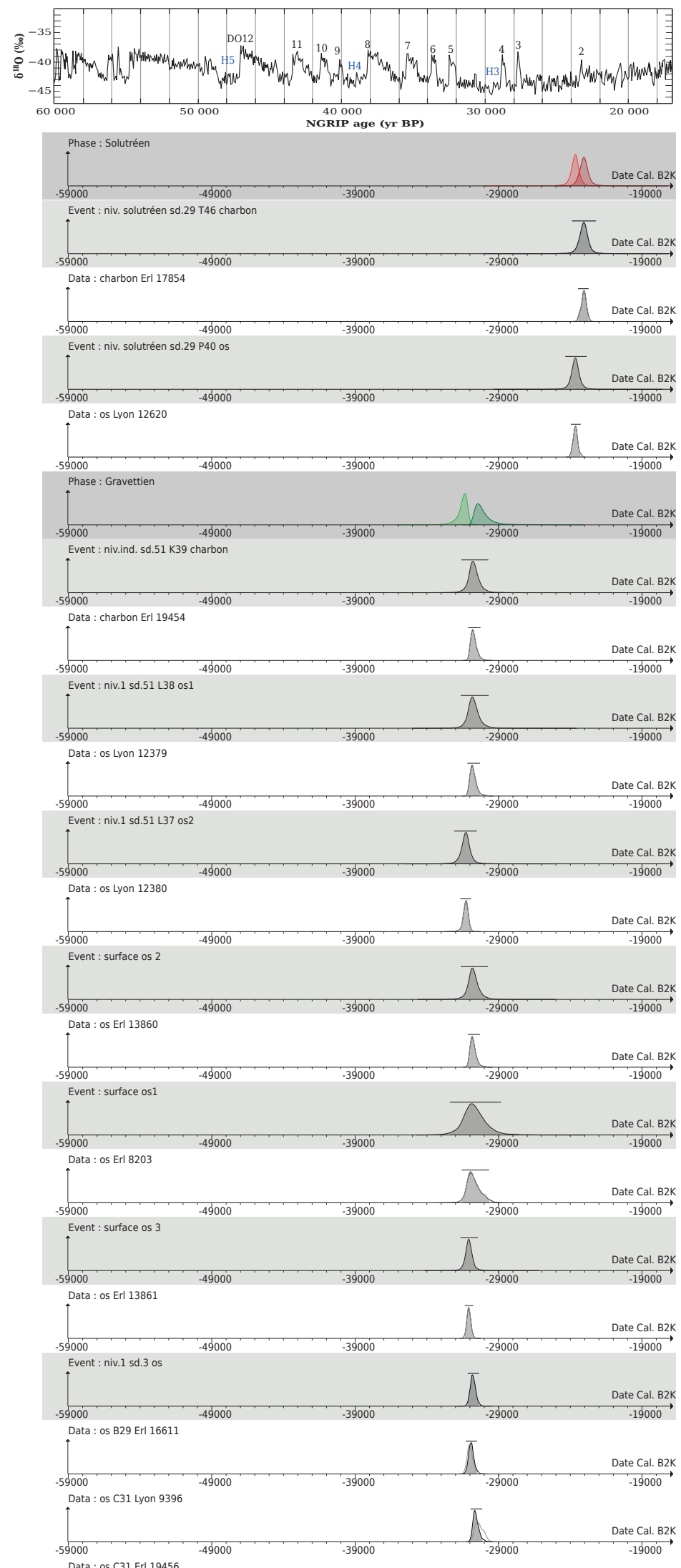

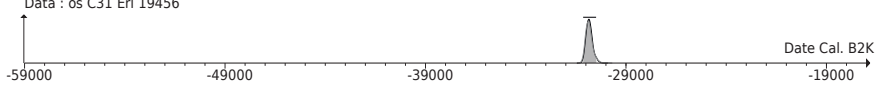

$\begin{array}{lllll}-59000 & -49000 & -39000 & -29000 & -19000 \\ -59000 & -49000 & -39000 & -29000 & -19000\end{array}$
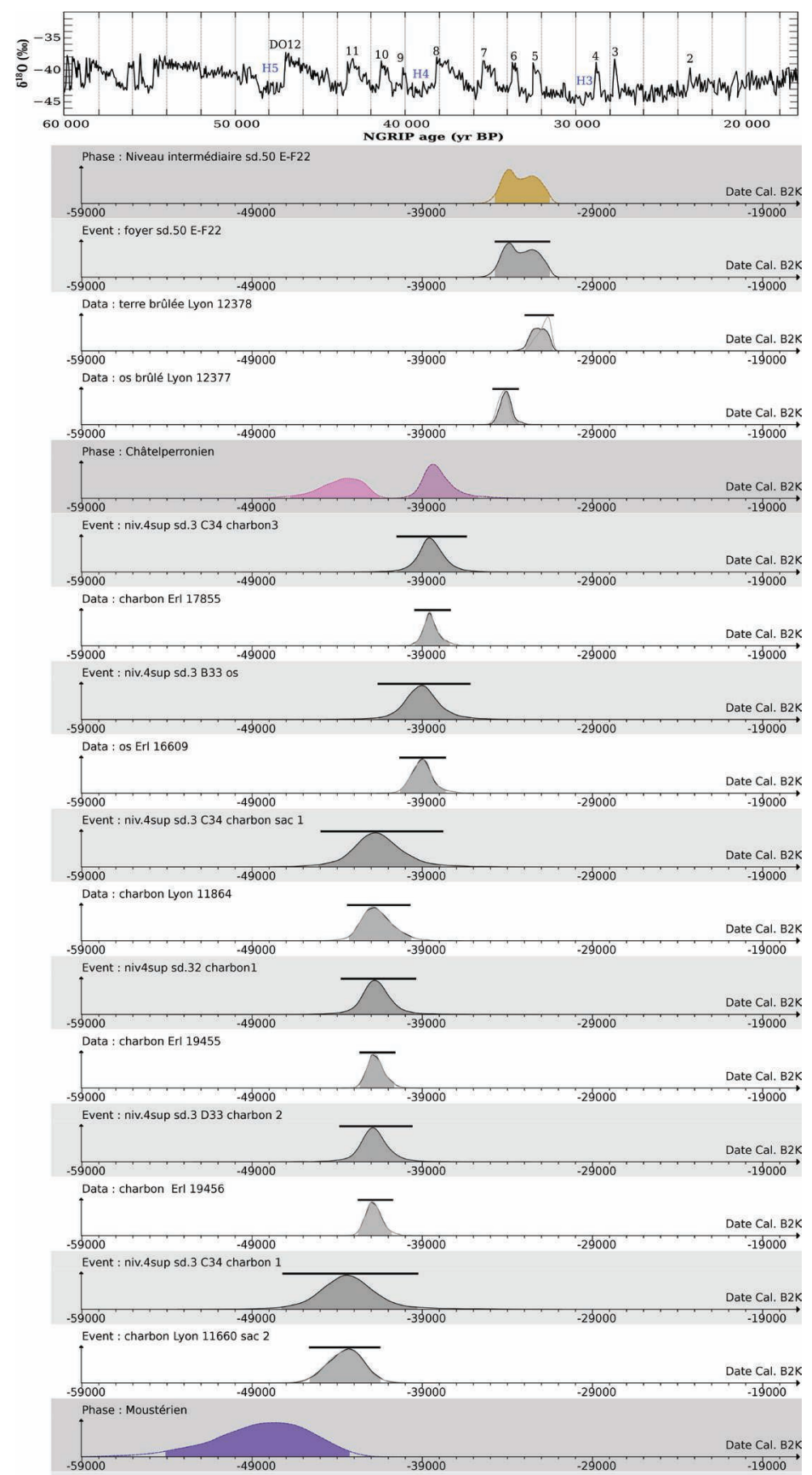

Event : foyer moustérien sd. 3 B
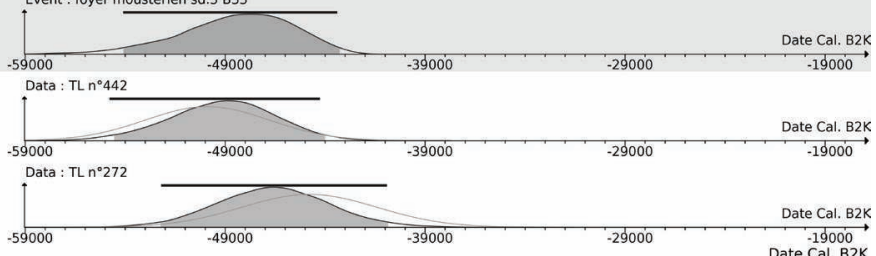

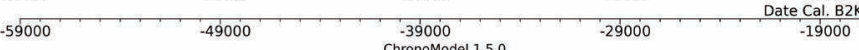

Tabl. IV

Tabl. III 
prélevés dans la nappe châtelperronienne. Ce résultat s'inscrit dans un intervalle de temps qui recouvre la plage chronologique d'une des datations du niveau intermédiaire, situé dans les loess à plus d'un mètre au-dessus du niveau 4sup. Cette date semble donc incohérente avec l'horizon châtelperronien et ne peut pas non plus être associée au niveau intermédiaire compte tenu de la position stratigraphique de l'os, ce pour quoi nous l'avons rejetée. Des modifications post-dépositionnelles attribuées à la carbonatation d'une partie des dépôts sableux et/ ou à l'activité biologique durant une éventuelle pédogenèse de cet ensemble sédimentaire pourraient expliquer ce rajeunissement (voir supra «Le contexte stratigraphique»). L'occupation châtelperronienne à Ormesson est ainsi située dans une période comprise entre 45768 et 37316 cal BP. Bien que les restes d'occupation remontant au Châtelperronien aient fait l'objet de nombreuses datations radiocarbone sur un grand nombre de sites, principalement en France, elles présentent une large gamme de résultats compris entre 45000 et $25000 \mathrm{BP}$ (entre 50000 et 28000 cal BP. c'est-à-dire au-delà des limites de la méthode pour les datations les plus anciennes) qui sont tant fonction des laboratoires que des échantillons datés. Les datations se concentrent principalement entre 45000 et 40000 cal BP (Zilhão et al., 2007). De nouvelles datations radiocarbones, réalisées sur une sélection d'objets en matière osseuse et d'os marqués de traces de découpe provenant des niveaux châtelperroniens de la Grotte du Renne à Arcy-sur-Cure, ont été récemment publiées (Higham et al., 2010). Compte tenu du grand nombre d'inversions des datations obtenues, les auteurs privilégient l'hypothèse de mélanges de matériel archéologique entre les différents niveaux individualisés lors des fouilles (du Moustérien au Proto-aurignacien) : «Our results confirm that material from several contexts has moved both up and down the stratigraphic sequence into the Châtelperronian levels».

Ce faisant, la stricte contemporanéité entre les restes humains néandertaliens et le mobilier lithique et osseux attribué au Châtelperronien est mise en doute et les auteurs de cet article préconisent, pour trancher définitivement, des datations directes sur les restes humains ainsi que sur les parures châtelperroniennes. Un article plus récent (Hublin et al., 2012) s'appuyant également sur de nouvelles datations effectuées sur du matériel osseux (os compact dont $35 \%$ présentent des traces d'action anthropique) des niveaux châtelperroniens de la Grotte du Renne, mais également sur un des ossements du squelette néandertalien de Saint-Césaire minimise le rôle des mélanges évoqués dans l'article précédent et attribue les industries lithiques et osseuses châtelperroniennes à l'homme de Néandertal. Les datations effectuées par l'équipe de J.-J Hublin donnent une fourchette comprise entre 40970 et 35500 BP (45240-39970 cal BP). On comprend bien que les positions concernant les datations des vestiges de la Grotte du Renne sont opposées dans leur conclusion respective quant à l'intégrité de la séquence stratigraphique et quant aux auteurs des industries lithique et osseuse châtelperroniennes et qu'il faudra documenter ces aspects anthropologiques à partir de gisements où les conditions de fouille et de conservation sont moins sujettes à caution. Le niveau châtelperronien d'Ormesson pourrait être de ceux-là vu sa surface potentielle et parce que les mélanges avec d'autres niveaux et notamment le niveau moustérien sous-jacent sont à l'évidence très limités et peuvent être contrôlés.

Le niveau châtelperronien d'Ormesson se trouve donc dans une fourchette chronologique relativement classique pour du Châtelperronien (Zilhão et al., 2007; Higham et al., 2010; Hublin et al., 2012; Higham et al., 2014; tabl. II). On retiendra cependant que cet intervalle chronologique est amené à être modifié au gré des découvertes et des nouvelles tentatives de traitement statistique des datations, car les modèles de phases chronologiques proposés jusqu'à présent font l'objet de discussions méthodologiques tant concernant l'échantillonnage des datations prises en compte dans les modèles que les hypothèses retenues lors de la modélisation bayésienne (Banks, 2015; Discamps et al., 2015).

\section{UN CHÂTELPERRONIEN DU NORD !}

S'il est un peu exagéré de qualifier le niveau châtelperronien d'Ormesson de Châtelperronien du nord, on ne peut que constater en effet que c'est le gisement situé le plus au nord en France et plus généralement en Europe (fig. 18). Les dernières synthèses sur le sujet (Roussel et Soressi, 2014) montrent que jusqu'en 2014, c'était en Bourgogne du Nord, la grotte du Renne et dans une bien moindre mesure celle du Bison à Arcy-sur-Cure, qui étaient les sites châtelperroniens les plus septentrionaux, pendant qu'en Angleterre, en Belgique ou en Allemagne ce sont d'autres technocomplexes qui caractérisent cette fameuse phase dite de transition tels que le Lincombien-RanisienJerzmanowicien (LRJ ; Flas, 2014). Un Moustérien tardif est aussi présent en Belgique autour de 40000-38000 BP (Pirson et Di Modica, 2011; Pirson et al. 2012). Le gisement les Bossats distant d'environ $130 \mathrm{~km}$ d'Arcy-sur-Cure, témoigne donc de l'extension du Châtelperronien vers le nord, montrant ainsi que ce groupe a fréquenté un espace géographique plus large que ce que l'on estimait jusqu'à présent. Comment se fait-il qu'il ait fallu attendre l'année 2014 pour s'en apercevoir? Il n'est pas impossible que le caractère relativement ubiquiste de l'industrie lithique n'ait pas permis d'identifier, plus au nord encore, des silex châtelperroniens, car il est vrai qu'en dehors des fameux couteaux, le lithique châtelperronien est assez peu caractéristique. Et ce d'autant plus si les schémas opératoires n'ont pas pu être reconstitués dans d'autres gisements et que l'on ne réfléchit qu'à partir d'objets indépendants. Ainsi les lames châtelperroniennes peuvent très bien être confondues avec des lames appartenant à d'autres complexes du Paléolithique supérieur, voire du Paléolithique moyen. L'agencement relativement facial du débitage laminaire châtelperronien peut, de plus, être aisément rapproché de débitages appartenant à d'autres traditions du Paléolithique supérieur, telles que le Solutréen ancien (Bodu et Renard, 2013a), le Magdalénien ancien (cf. l'industrie lithique du gisement de Thèmes, Yonne, Malgarini et al., 2017) ou encore l'Azilien ancien (Bodu et Mevel, 2008). En l'absence du fossile directeur qu'est la pointe de Châtelperron, qui peut d'ailleurs parfois être confondue avec des pointes aziliennes par exemple, il n'est donc pas impossible que certaines industries lithiques châtelperroniennes de la partie nord de la France aient été mal diagnostiquées.... 
La situation très au nord de ce gisement permet de poser ce faisant la question d'une extension encore plus septentrionale du phénomène châtelperronien et des relations entre les gisements de transition (LRJ) situés les plus au «sud», en Belgique (Goyet, Spy, Couvin) et en Angleterre (Flas, 2014).

\section{INFORMATIONS SUR LA SÉRIE LITHIQUE CHÂTEL- PERRONIENNE D'ORMESSON}

Dans le sondage 50, 295 silex taillés hors esquilles ont été relevés. Le sondage 3 a livré 1267 silex hors esquilles. Notre réflexion porte donc sur un peu plus de 1500 pièces d'une taille supérieure à $1 \mathrm{~cm}$. Dans le sondage 3, la panoplie lithique est dominée quantitativement par les éclats (éclats, éclats laminaires et lamellaires) avec près de $56 \%$ du corpus. Mais les parts du laminaire $(12,5 \%)$ et du lamellaire $(24,8 \%)$ sont loin d'être négligeables et correspondent au contraire aux intentions du débitage, en tout cas pour les premières. La démonstration peut en être faite à partir de la lecture des nucléus au nombre de 6 (fig. 19), dont aucun ne montre de véritable intention de production autonome d'éclats y compris un bloc dont les derniers négatifs sont des éclats, mais qui font suite à une production de lames. Au contraire, les nucléus du sondage 3 et 50 et un burin nucléiforme du sondage 50 montrent soit une exploitation vouée à la production de lames, soit à celle de lamelles, même si certains de ces nucléus à lamelles témoignent d'un savoir-faire médiocre et d'une régularité tout aussi moyenne des produits obtenus (fig. 20). Mais c'est surtout la très faible implication des éclats dans l'outillage qui montre qu'ils ne constituent pas un réel objectif des débitages. Cela n'exclut pas toutefois qu'une forme appropriée d'éclat ait pu intéresser un utilisateur comme c'est le cas d'un éclat retouché en couteau de Châtelperron (voir supra). En effet sur les 44 outils, les lames représentent près de $60 \%$ de l'outillage (26), les éclats (3) n'en constituant qu'un peu moins de $7 \%$ et les éclats laminaires ou lamellaires au nombre de 9, environ $20 \%$, mais ces supports sont à l'évidence des sous-produits des débitages laminaire et lamellaire, le premier acceptant une certaine diversité dans la régularité des produits obtenus.

Dans le sondage $50^{8}$, les éclats, quelle que soit leur morphologie, dominent l'ensemble avec près de $60 \%$, mais il s'agit, pour l'essentiel, de produits de mise en forme des blocs et d'entretien des volumes. Les lames $(12,5 \%)$ et plus encore les lamelles $(22,7 \%)$ sont également bien représentées. Aucun des 3 nucléus du sondage 50 ne témoigne d'une production d'éclats autonomes. Deux sont des nucléus à lamelles ou produits d'apparence lamellaire, l'un sur éclat, l'autre sur fragment de bloc, le troisième est un nucléus à lames, les derniers produits utiles obtenus mesurant environ $70 \mathrm{~mm}$ de longueur (fig. 21). L'identification des supports de l'outillage est moins convaincante que pour le matériel du sondage 3 .

8. La concentration de matériel lithique du sondage 50 s'étend sans doute bien au-delà des deux mètres carrés fouillés! La quantité de

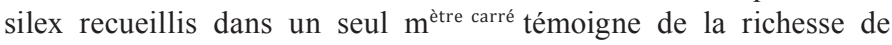
l'endroit, ce qui plaide en faveur de l'existence d'une seconde concentration avec celle du sondage 3 .
On ne compte en effet que 5 outils sur 22 objets affiliés à l'outillage, parmi lesquels les chutes de burin et esquilles de retouche d'outils dominent. Les 5 outils sont fabriqués sur tous les types de supports, éclats (2), éclat lamellaire (1), lame (1), lamelle (1), mais il est évident que leur faible nombre n'est en rien représentatif et il faudra attendre une fouille plus conséquente pour mieux identifier la répartition de l'outillage en fonction des types de supports.

\section{UNE PRODUCTION DE SUPPORTS LAMINAIRES}

Les lames sont donc les produits recherchés pour l'essentiel des supports de l'outillage peu diversifié en termes de types, couteaux de Châtelperron et burins. Le schéma opératoire désormais classique du débitage laminaire mis en évidence par Jacques Pelegrin sur les sites de Roc-de-Combe et de la Côte (Pelegrin, 1995), confirmé et précisé par la suite par d'autres chercheurs (Grigoletto et al., 2008; Bachellerie, 2011 ; Bordes et Teyssandier, 2011 ; Roussel, 2011 ; Aubry et al., 2012 et 2013), s'applique parfaitement à la série châtelperronienne d'Ormesson. De nombreux raccords et remontages (36\% pour le sondage 3 et un peu plus de $10 \%$ pour le sondage 50 ) ont permis de documenter de façon très précise la méthode de production des lames et, avec moins de détails, celle des lamelles (fig. 22).

Des blocs de silex du Campanien affleurant à environ $5 \mathrm{~km}$ vers l'est ont été exploités (Denizot, 1971). Rognons choisis de morphologie allongée et relativement plats aux cortex lessivés et/ou roulés, ils ont vraisemblablement été collectés dans les alluvions du Loing. Ils présentent une longueur moyenne de $15 \mathrm{~cm}$ et dépassent rarement $20 \mathrm{~cm}$. Le débitage commence par une partie étroite du nodule avec l'extraction de lames et d'éclats laminaires le long d'un relief cortical ou d'une crête uni ou bifaciale préalablement installée et il investit progressivement la largeur du bloc avec le détachement de lames plus minces et plus larges (fig. 21, fig. 23). Cette étape d'entame des blocs a l'avantage de permettre au tailleur de créer assez rapidement et facilement des nervures prononcées dans la longueur du nodule en raison de l'étroitesse de la première surface investie. Ces premières nervures vont guider les enlèvements suivants qui quittent progressivement la «tranche» pour investir une surface plane. Quelques remontages montrent clairement cette extension du débitage de la tranche vers une face large, c'est notamment le cas des nucléus E20.135 et C34.228 (fig. 21, fig. 22). C'est donc la partie naturelle la plus étroite du rognon qui est privilégiée pour débuter les opérations, le débitage tournant ensuite vers une voire plusieurs surfaces larges du nucléus. C'est en quelque sorte un débitage semi-tournant «définitif» avec un choix très net porté vers l'extraction des lames sur les surfaces planes afin d'obtenir des supports plans, à la nervure centrale peu prononcée et dont l'un des pans est également large, les supports idéaux des couteaux de Châtelperron. Le terme définitif est ici utilisé pour bien insister sur ce choix des châtelperroniens d'investir les surfaces planes sans retour véritable vers les surfaces les plus cintrées si ce n'est pour quelques recadrages de la surface laminaire au cours de l'exploitation du bloc. La morphologie des nucléus 

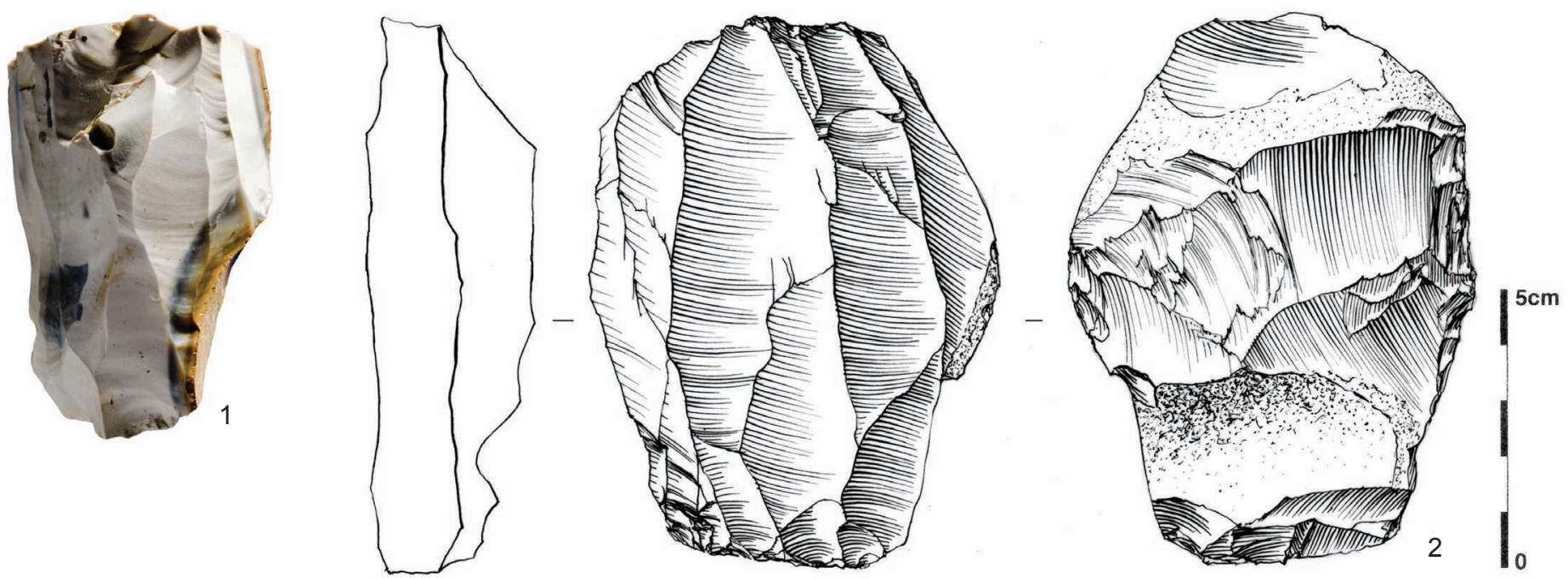

Fig. 19 - Deux nucléus laminaires du sondage 3 (C) : P. Bodu, CNRS et DAO : M. Ballinger, CNRS).

Two blade cores from test-pit 3 (С: P. Bodu, CNRS \& CAD: M. Ballinger, CNRS).
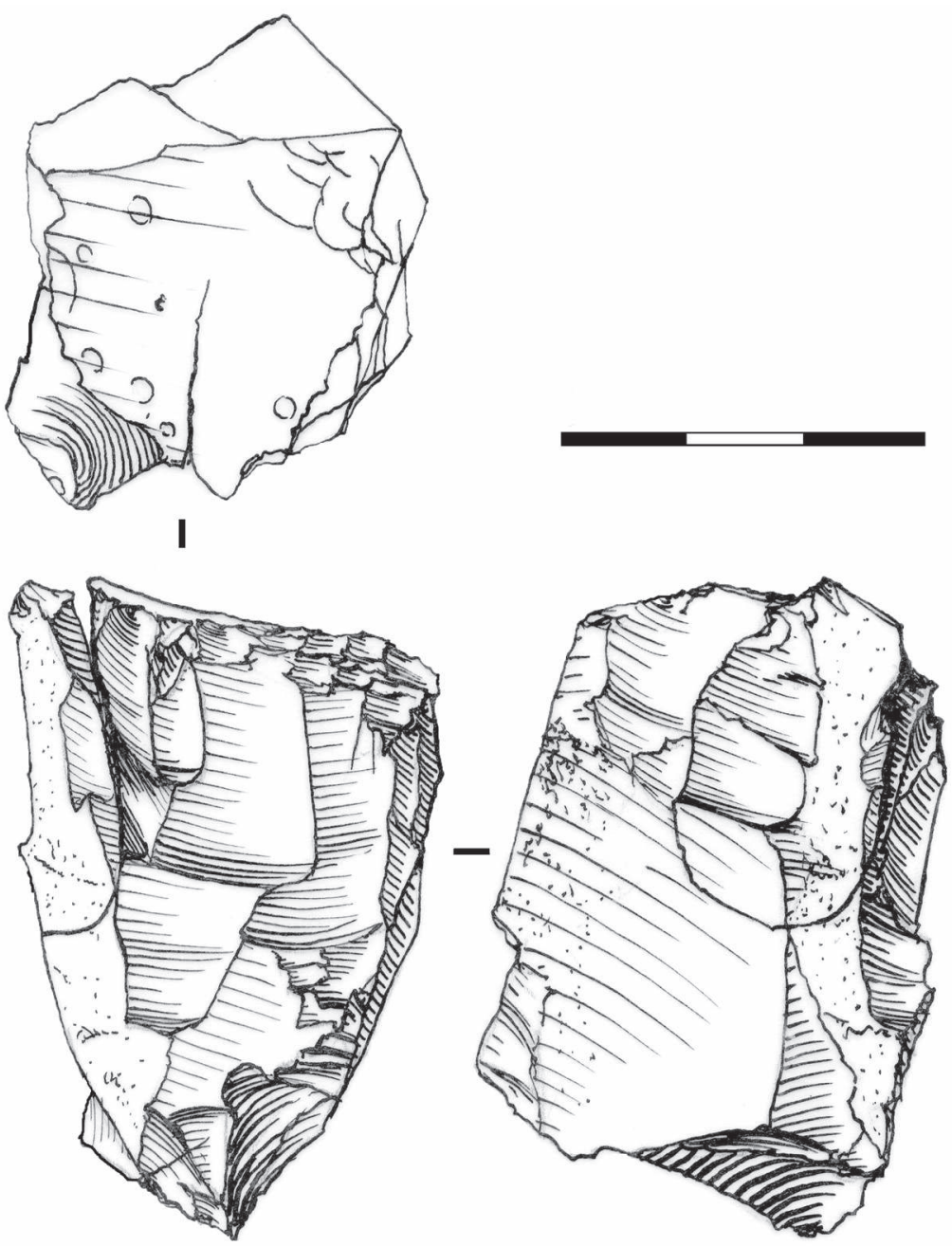

Fig. 20 - Nucléus à exploitation maladroite du sondage 50 (DAO : M. Ballinger, CNRS). 
Fig. 21 - Un nucléus laminaire du sondage 50 (à gauche) et son remontage (à droite ; (C): P. Bodu, CNRS et DAO : M. Ballinger, CNRS).

A blade core from test-pit 50 (on the left) and its refitting (on the right; $\odot$ : P. Bodu, CNRS \& CAD: M. Ballinger, CNRS).
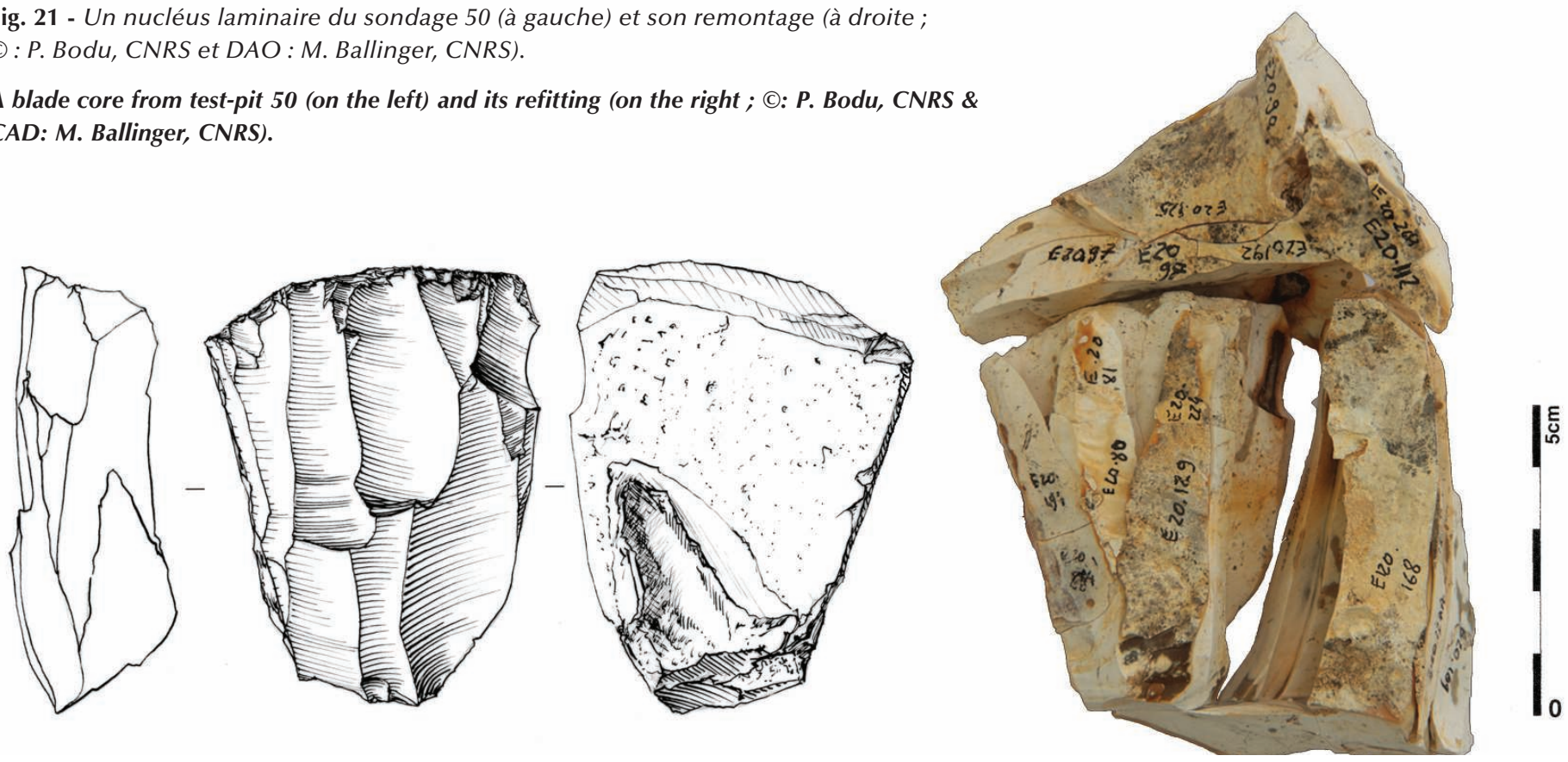

Fig. 22 - Deux débitages laminaires partiellement remontés du sondage 3 (C): P. Bodu, CNRS, DAO : M. Ballinger, CNRS).

Two blade reduction sequences, partially refitted, from test-pit 3 (@): P. Bodu, CNRS \& CAD: M. Ballinger, CNRS).

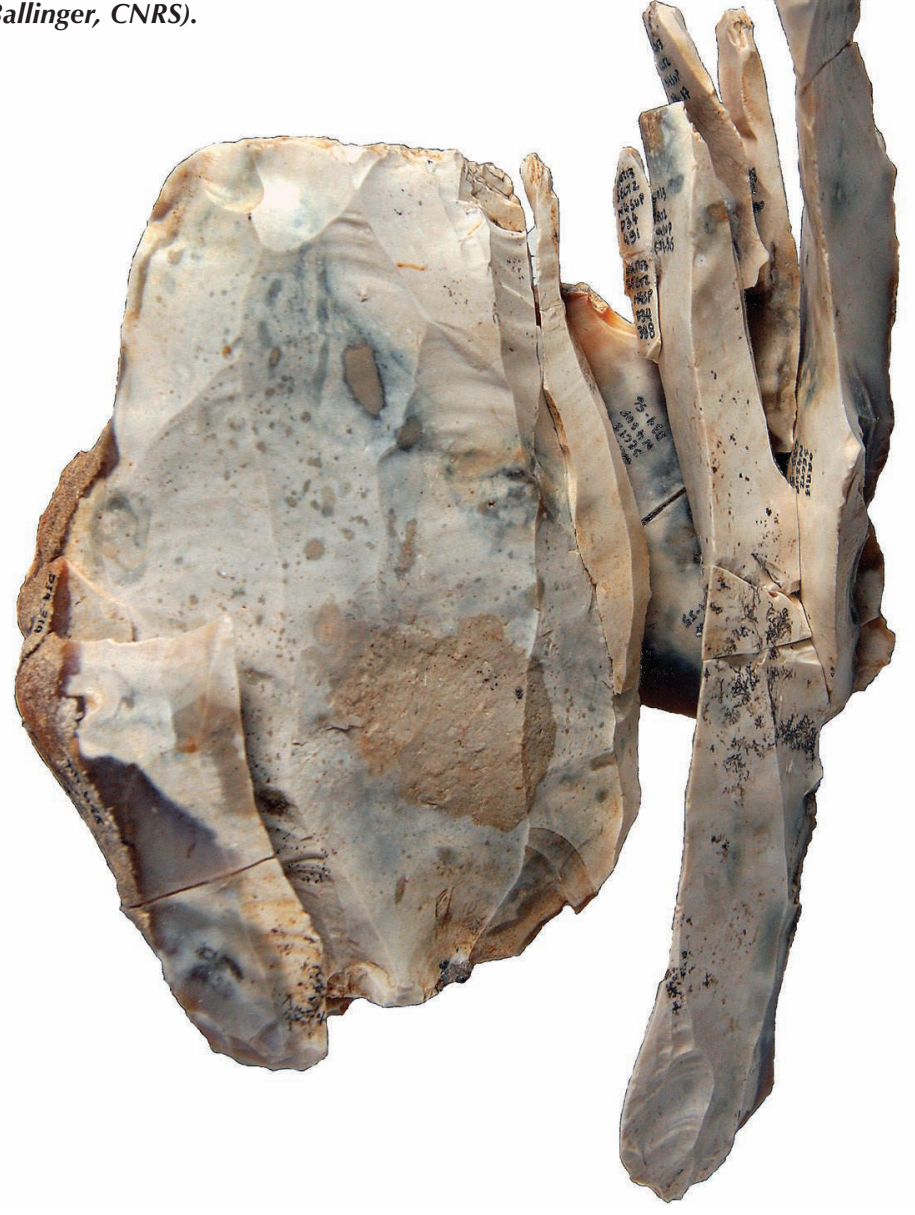




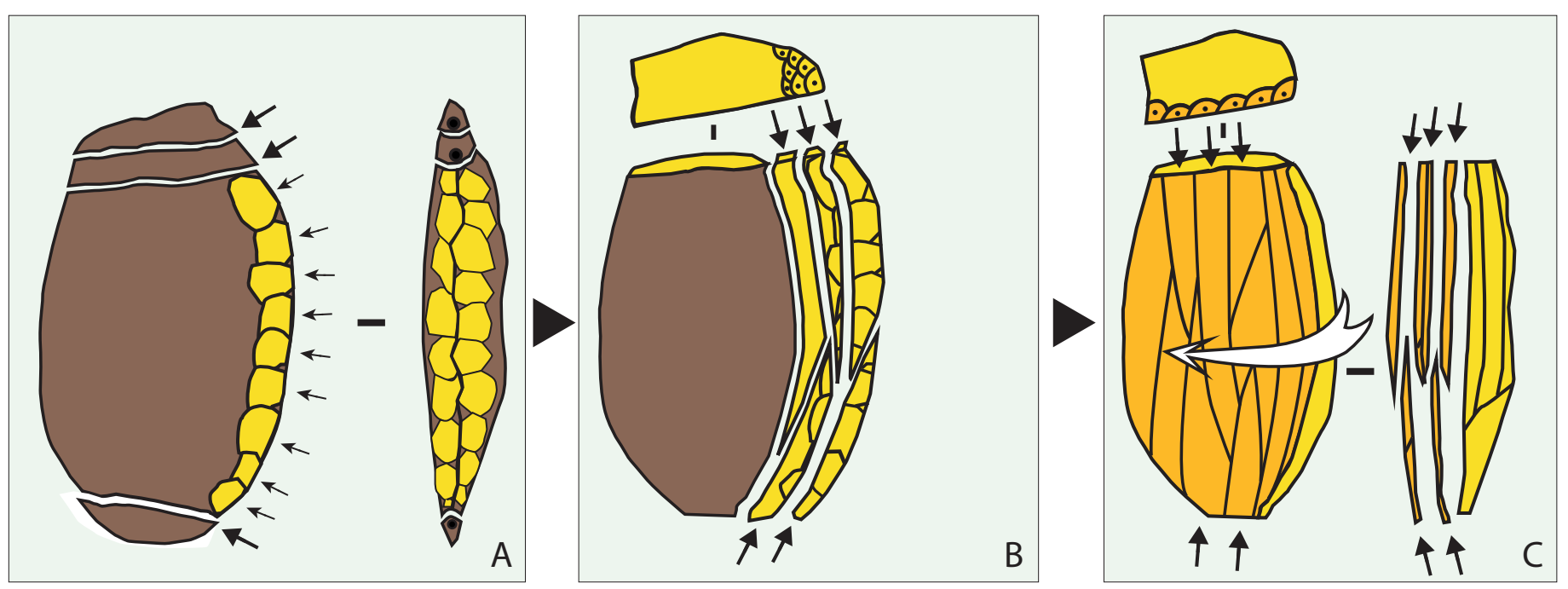

Fig. 23 - Schéma simplifié d'un débitage châtelperronien (DAO: M. Ballinger, CNRS).

Schematic drawing of a Châtelperronian debitage sequence (CAD: M. Ballinger, CNRS).

à l'abandon témoigne très clairement de cette recherche de platitude (fig. 19, fig. 21, fig. 22).

Dans plusieurs ensembles remontés, on observe que deux plans de frappe sont installés très rapidement de part et d'autre de la future surface laminaire. C'est notamment le cas de deux de ces blocs, découverts dans les sondages 3 et 50, respectivement les blocs $\mathrm{C} 34.228$ et le bloc E20.155 (fig. 22 et fig. 21) où les deux plans de frappe sont installés quasiment au même moment, dès le début de l'exploitation. Les lames sont débitées à partir d'un plan de frappe ou de l'autre, au gré de l'évolution de la table, l'usage de la pierre tendre autorisant et nécessitant cette bipolarité (fig. 24). Un plan de frappe apparaît cependant préférentiel si l'on en juge par le déséquilibre numérique qui existe dans la production de lames extraites entre les deux plans de frappe. L'autre intérêt des remontages sur le matériel d'Ormesson est la démonstration de l'utilisation de longs plans de frappe étroits parallèles à la table laminaire finale qui assurent la progression du débitage laminaire de la face étroite du nucléus vers une face large. En installant ainsi très vite ces plans de frappe oblongs, le tailleur se donne la possibilité d'investir la face large sans avoir à remanier de façon trop importante la surface de plan de frappe. La démonstration la plus tangible, en dehors des remontages, est sans doute le faible nombre d'éclats ou de tablettes de réaménagement de plan de frappe dans l'ensemble lithique recueilli dans les deux sondages. Une nette tendance à l'outrepassage a été observée sur un grand nombre de supports laminaires. Sans négliger l'hypothèse qu'il s'agisse là de la conséquence de coups portés un peu trop fort par rapport à la longueur du nucléus et donc d'accidents de taille, il nous semble que la répétition de ces outrepassages et leur forte intégration dans le schéma de débitage correspondent à une véritable intention, un procédé destiné à entretenir la surface laminaire et peut-être à favoriser la création de nervures sur lesquelles vont filer les supports des couteaux de Châtelperron notamment (fig. 21). Les produits laminaires, et lamellaires également, sont donc détachés par percussion directe à la pierre tendre si l'on en juge par la taille réduite de certains talons, l'esquillement du bulbe et les ondes fines et serrées visibles sur la face inférieure de nombreuses lames et lamelles, dans d'autres cas un bulbe particulièrement mince et bien détouré et enfin le profil rectiligne des supports (Pelegrin, 2000 ; fig. 24). Bien qu'aucun percuteur n'ait été retrouvé, on privilégie l'emploi d'un grès, matière première locale qui ne devait pas faire défaut. Ce dernier est utilisé selon différentes modalités : soit en percussion en retrait à l'intérieur du plan de frappe sur des surfaces lisses ou facettées qui donne des talons larges en particulier pour les produits d'installation et d'entretien des surfaces, soit en percussion près du bord de plan de frappe pour les produits de première intention. Ce sont

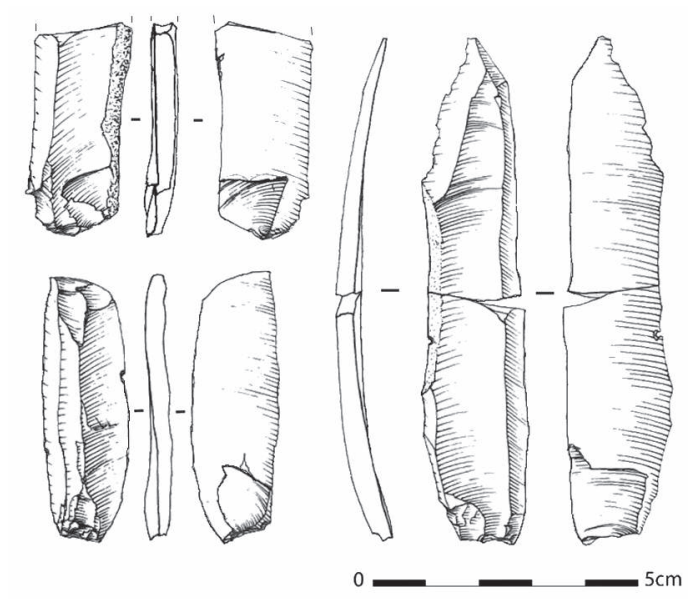

Fig. 24 - Faces inférieures de lames témoignant de l'usage de la pierre tendre pour leur débitage (DAO: M. Ballinger, CNRS).

Ventral sides of blades showing evidence of the use of soft stone hammers for blank production (CAD: M. Ballinger, CNRS). 
alors des talons minces, linéaires ou punctiformes, parfois éclatés, présentant une corniche soigneusement réduite et abrasée qui sont révélateurs de cette modalité de percussion. Ce geste de préparation ou encore le caractère rectiligne des lames n'ont rien à envier à des préparations du bord de plan de frappe ou des morphologies de lames observées dans d'autres industries lithiques plus récentes du Paléolithique supérieur. C'est notamment ce qui nous amène à penser que l'industrie châtelperronienne, lorsqu'elle est trouvée hors contexte stratigraphique bien défini et qu'elle ne présente pas de couteaux de Châtelperron, pour des raisons économiques et d'activités, est peu facile à diagnostiquer. Le Châtelperronien est donc éminemment d'essence Paléolithique supérieur en ce qui concerne son industrie lithique, mais cela a-t-il un sens de l'écrire alors qu'on sait qu'au sein du Paléolithique moyen, dans certains complexes moustériens on a taillé des lames (Révillon, Tuffreau, 1994) et à l'inverse que de rares industries du Paléolithique supérieur comme le Badegoulien, ont redécouvert le débitage d'éclats (cf. Cretin, 1996; Morala, 1993; Bodu et al., 2007)?

\section{UN INTÉRÊT ÉVIDENT POUR LES LAMELLES}

La présence d'une production lamellaire au Châtelperronien est désormais un fait avéré (Pelegrin, 1995; Bachellerie, 2011; Roussel, 2011). Si les modalités de production sont parfois décrites, il est en revanche plus difficile de connaître le ou les objectifs de cette production tant les lamelles retouchées sont rares, à l'exception du site de Quincay (Rousssel, 2011; Roussel et al., 2016) où la présence de grandes lamelles Dufour sous-type Dufour ou un autre type, ne fait aucun doute selon
M. Roussel. La découverte de nombreuses lamelles, pour l'essentiel non retouchées, à Ormesson, représente dès lors une opportunité supplémentaire pour discuter de l'intérêt que les châtelperroniens ont accordé à ce type de support. Dans le sondage 3, les lamelles fortement représentées en tant qu'éléments bruts, n'interviennent que de façon très limitée dans l'outillage puisque seules 4 lamelles sur les 314 lamelles entières ou fragments, portent des retouches d'aménagement ou d'utilisation évidentes, une petite dizaine d'autres montrant des esquillements ou des encoches répartis de façon trop aléatoire pour être considérés assurément comme des retouches intentionnelles ${ }^{9}$ (fig. 25). Malgré cela leur forte quantité (314), plaide en faveur d'une production volontaire et ce d'autant plus que quelques rares lamelles brutes portent des traces d'utilisation ou d'emmanchement ${ }^{10}$ et que des nucléus témoignent d'une production lamellaire ou en tout cas de supports allongés étroits (3 sur les 7 nucléus identifiés). Deux sont des nucléus sur éclat épais dont l'exploitation débute par la tranche et envahit pro-

9. L'ensemble des produits lamellaires numérotés ou provenant des esquilles voire des refus de tamis ont été observés à la loupe binoculaire et pour certaines au microscope.

10. Trois lamelles brutes présentent sur leur arête dorsale un biseau de poli non orienté correspondant à une matière dure indéterminée. Si deux d'entre elles sont intactes sur leurs bords tranchants, la troisième présente une cassure en flexion de la quasi-totalité d'un bord ainsi que, sur le bord opposé, de légers esquillements bifaciaux non diagnostiques. Sa partie apicale est cassée transversalement de façon non diagnostique. Les polis sur ces trois lamelles brutes peuvent être mis en relation avec des polis similaires présents également sur l'arête dorsale de deux lamelles retouchées (une retouchée de façon continue et une présentant une coche retouchée).
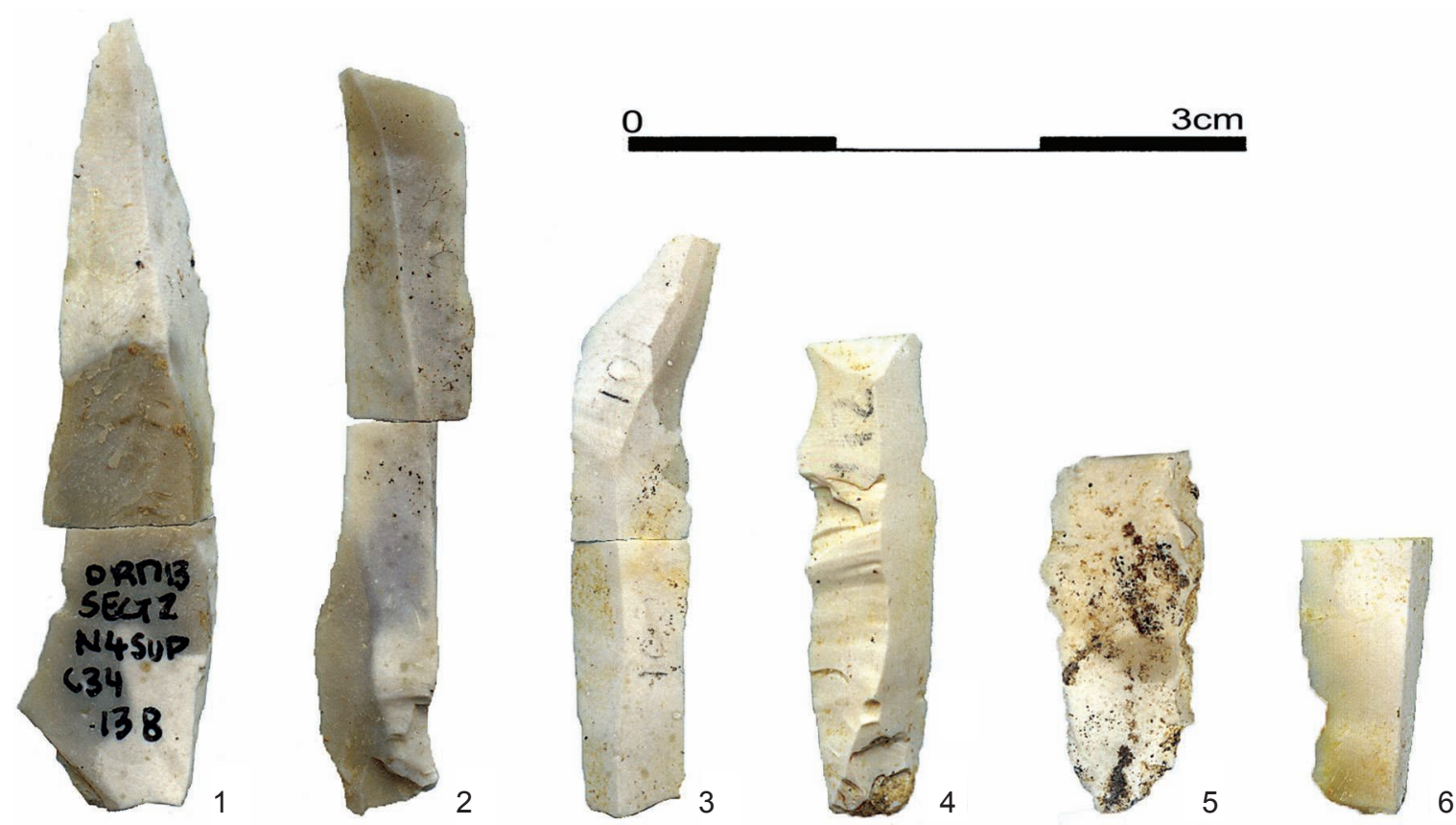

Fig. 25 - Des lamelles brutes du niveau châtelperronien (DAO : M. Ballinger, CNRS et (C) : P. Bodu, CNRS).

Unretouched bladelets from the Châtelperronian level (CAD: M. Ballinger, CNRS \& $\odot:$ P. Bodu, CNRS). 
gressivement la face inférieure (ensemble 7, nucléus D33.201, fig. $26, \mathrm{n}^{\circ} 1$; nucléus $\mathrm{D} 34.834$, fig. $26, \mathrm{n}^{\circ} 2$ ), le troisième est une petite corne corticale détachée accidentellement d'un rognon de taille moyenne (environ $15 \mathrm{~cm}$ ), qui a donné des petites lames ne pouvant pas dépasser $5,5 \mathrm{~cm}$ de longueur et entre lesquelles s'intercalent des lamelles (ensemble 3, nucléus C33.14 ; fig. 27). De petits supports de débitage ont donc été choisis, éclats épais comme corne corticale pour l'obtention de supports lamellaires. Dans le sondage 50, les lamelles sont également nombreuses (67 soit 22,7\%), mais une d'entre elles seulement porte de nettes retouches intentionnelles de cadrage de bord (fig. 28b), alors que quelques-unes portent de fins esquillements sur un bord dont l'origine est délicate à préciser (fig. 28a). Cette grande lamelle retouchée présente sur le bord brut opposé un poli de découpe de matière tendre organique, possiblement d'origine animale. Sur la ligne de fracture proximale, côté face inférieure, a été observé un poli de matière dure organique, nettement orienté dans le sens d'allongement de la lamelle, évoquant au premier abord un poli de matière animale ${ }^{11}$. Par ailleurs, dans le sondage 50 un nucléus sur tranche d'éclat (ou burin transversal épais sur troncature), identique dans son exploitation initiale à celui du sondage 3, a livré également quelques rares lamelles (ensemble 14, nucléus E20.54 ; fig. 26, $\mathrm{n}^{\circ} 3$ ). Bien que l'on ne puisse exclure la participation d'un certain nombre de lamelles aux opérations d'entretien des convexités de la table (le cintrage du haut de la surface laminaire par exemple), la régularité de nombre d'entre elles (bords parallèles, rectitude, nervure centrale), une préparation soignée des talons (minces, lisses à corniche abrasée), sont autant d'arguments en faveur d'une production intentionnelle. Cette constatation faite tant dans le sondage 3 que dans le sondage 50 est d'autant plus importante que l'existence d'une production lamellaire dans le Châtelperronien n'allait pas de soi jusqu'à il y a une dizaine d'années avant les démonstrations faites à partir des gisements du Basté, de Bidart ou de Canaule II (Bachellerie, 2011) et de Quincay (Roussel, 2011). Auparavant, des productions lamellaires avaient été identifiées dans les sites de Roc-de-Combe, couche 8 et La Côte, niveau 3 (Pelegrin, 1995) : «L'existence d'un débitage intentionnel de lamelles est attestée à la Côte III, et très probable à Roc-deCombe c.8, même si les lamelles retouchées sont rares» (p. 253). L'auteur n'écartait pas alors la possibilité à Roc-deCombe de l'existence de mélanges entre le matériel châtelperronien et celui des couches aurignaciennes sus-jacentes (p. 181), en particulier pour des lamelles Dufour découvertes dans la couche 8 et soupçonnées d'être plus récentes. Mais dans le cas de Roc-de-Combe, la quantité de lamelles et l'existence de nucléus dédiés apparemment à une production lamellaire plaidaient, selon l'auteur, plutôt en faveur d'une véritable produc-

11. Mais des plages de poli plat se trouvent parfois mêlées à ce poli vers la face inférieure. Étant donné qu'elles ne se trouvent nulle part ailleurs, elles pourraient signer un emmanchement en contrainte contre un élément dur organique, mais l'absence d'esquillements caractéristiques tempère fortement cette hypothèse. Dès lors, la piste d'une altération taphonomique ponctuelle n'est pas à exclure, bien que peu probable. tion lamellaire châtelperronienne. Une des conclusions sur cet aspect témoignait néanmoins de la prudence que J. Pelegrin assortissait à ses observations et aux hypothèses qui en découlaient : «Outils, restes de taille bruts et nucléus apportent ainsi des présomptions convergentes... Mais une étude scientifique n'est pas un procès et ne peut reposer sur une conviction. La mienne reste ainsi dans l'incertitude. Si le débitage intentionnel (au moins opportuniste) de lamelles régulières paraît probable, il n'est pas prouvé» (p. 181). Cette prudence semblait tout à fait de mise à une époque où la description des schémas opératoires du débitage châtelperronien se mettait véritablement en place et en raison en effet des risques de mélanges susceptibles d'avoir affecté les niveaux archéologiques incriminés à Roc-de-Combe notamment. Depuis, les travaux de M. Roussel et F. Bachellerie ont permis de confirmer les bonnes observations de J. Pelegrin même si le constat est fait de la faible présence des lamelles dans les gisements châtelperroniens en général et du caractère exceptionnel des éléments lamellaires retouchés. Des lamelles certes, mais pour quoi faire? À Canaule II, il est conclu que «malgré sa discrétion, l'existence d'un débitage de supports microlithiques allongés, réguliers et aux nervures parallèles, est indiscutable» (Bachellerie, 2011, p. 291). Dans ce dernier travail, les termes de petites lames remplacent parfois ceux de lamelles ou encore de supports microlithiques allongés. Cela montre la difficulté qu'il y a à instaurer une véritable distinction entre ces deux catégories qui dans bien d'autres technocomplexes du Paléolithique supérieur sont bien séparées ne serait-ce que par leur morphométrie et leur fonction et surtout la difficulté à identifier la réalité de la production lamellaire châtelperronienne : «Ces éléments restent toutefois très discrets et difficilement dissociables du débitage laminaire.» (Bachellerie, 2011, p. 25). C'est une discussion du même ordre qui est développée au sujet de l'étude du matériel lithique châtelperronien des Tambourets (Couladère, Haute-Garonne ; Scandiuzzi, 2008), puisque l'auteur choisit de nommer petites lames, des supports qu'ailleurs on pourrait appeler lamelles, «...afin d'éviter toute confusion avec des produits bien décrits et documentés par nombre de chercheurs (Le Brun-Ricalens et al., 2005), et répondant à des critères technologiques et morphométriques précis». Les largeurs d'un bon nombre de négatifs des derniers produits obtenus sur les nucléus, se rapprochent en effet plus du standard de ce que l'on appelle lamelle, que de la lame. C'est d'ailleurs le terme lamelle qui est utilisé en légende de la figure 12 (Scandiuzzi, 2008, p. 37), qui illustre des nucléus sur «tranche d'éclats». Pour le gisement de Basté, l'absence de production lamellaire avérée semble s'expliquer en grande partie par les conditions de récolte du matériel et notamment «l'imprécision du tamisage» (Bachellerie, 2011, p. 141), mais aussi par des conditions de formation et d'évolution des niveaux archéologiques peu favorables à la conservation des petits éléments lithiques. Certes, «l'existence d'un débitage de supports allongés et réguliers, aux nervures parallèles et de moins de $50 \mathrm{~mm}$ de long, semble indiscutable» aux yeux de F. Bachellerie (p. 142), mais ce dernier reste prudent quant à la présence d'une véritable production lamellaire autonome au Basté en raison notamment de «l'absence de supports microlithiques retouchés». Le constat est le même 

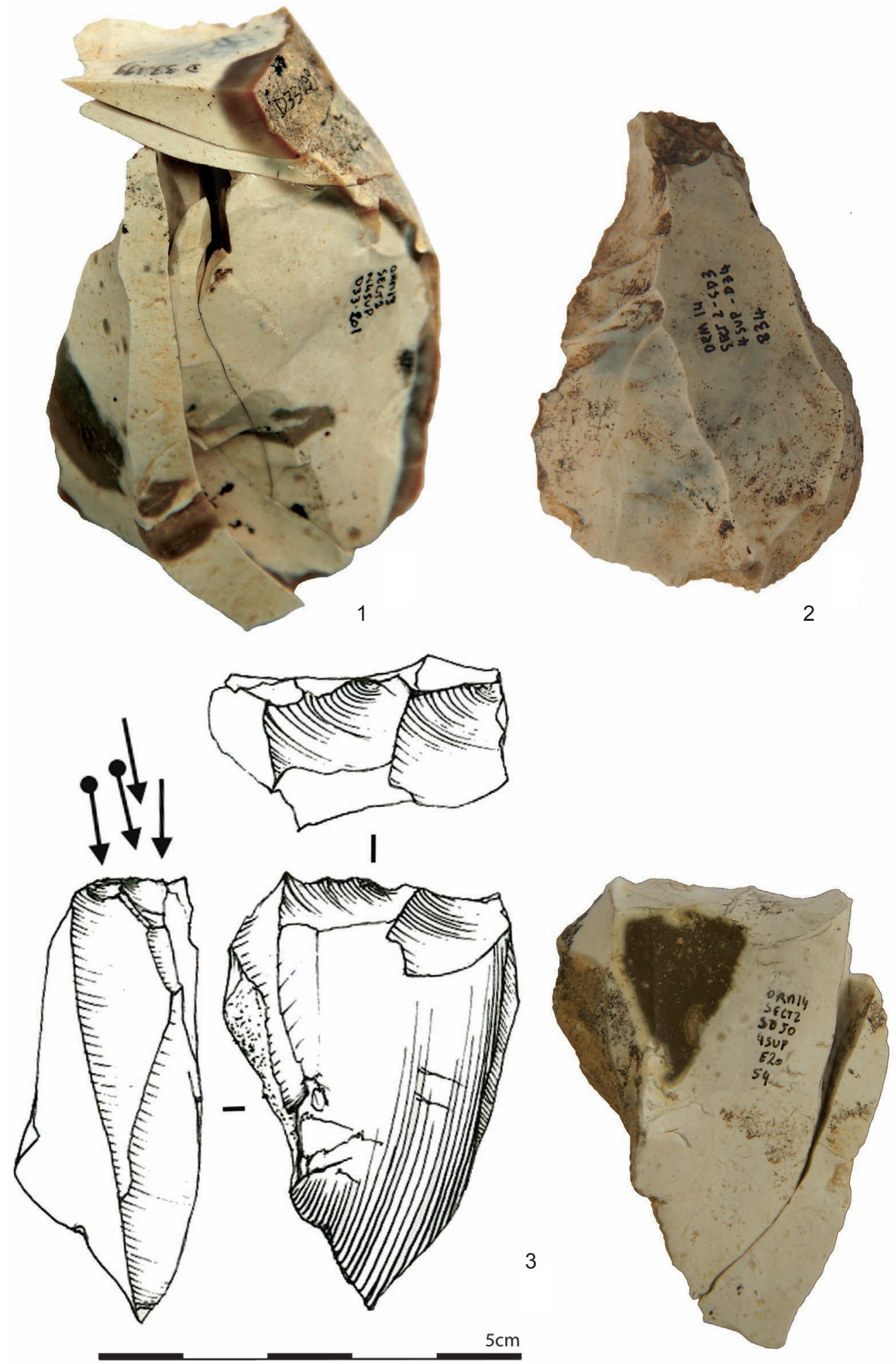

Fig. 26 - Trois nucléus à lamelles sur éclat épais des sondages $3(1,2)$ et 50 (3; (C) : P. Bodu, CNRS et DAO : M. Ballinger, CNRS). Three bladelet cores on thick flakes from test-pits $3(1,2)$ and 50 (3; @: P. Bodu, CNRS \& CAD: M. Ballinger, CNRS). 


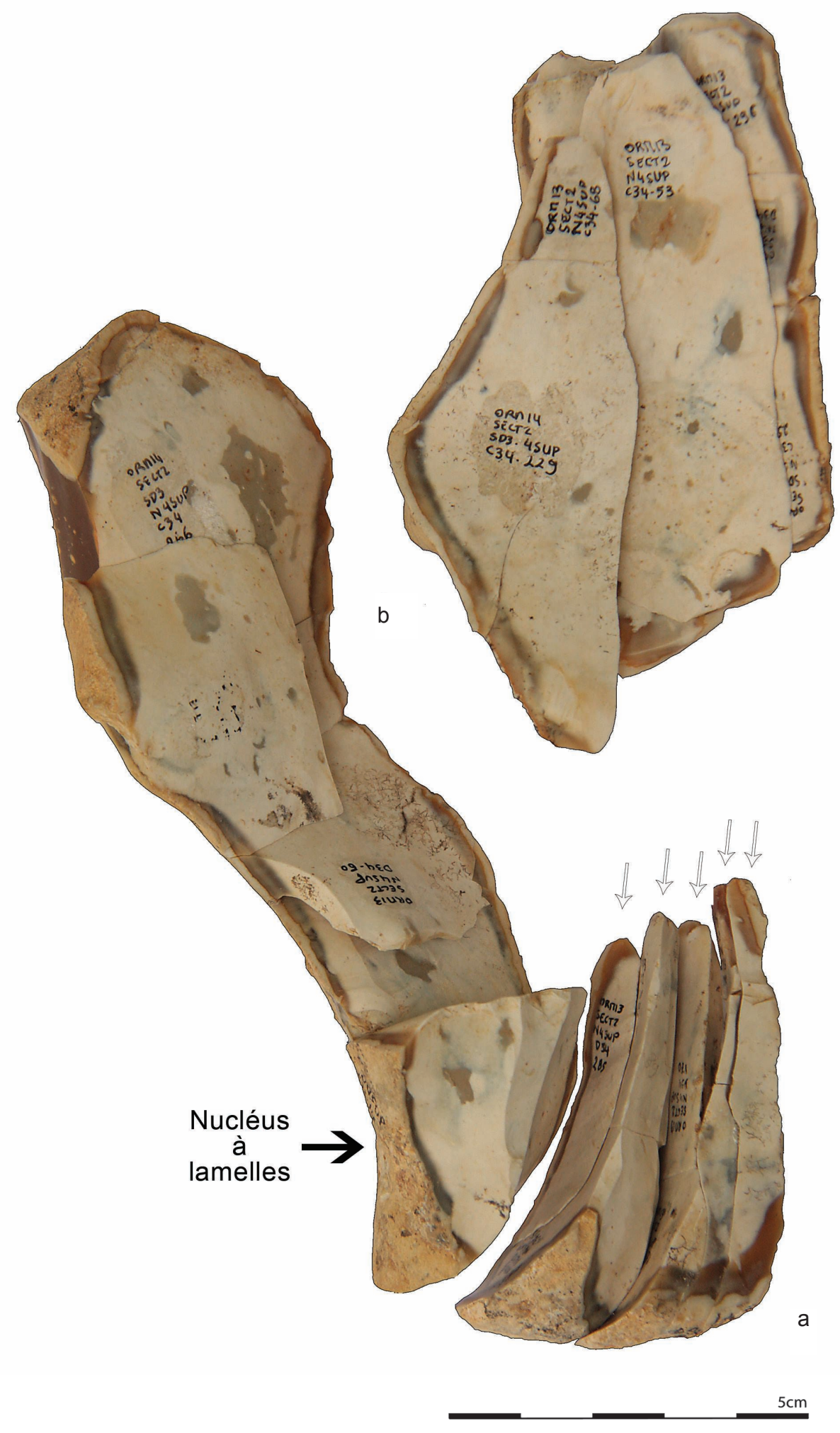

Fig. 27 - Remontage d'un nucléus à lamelles sur corne corticale (a) appartenant à un bloc de silex sur lequel des lames ont été débitées ( $b$; ( $:$ P. Bodu, CNRS et DAO : M. Ballinger, CNRS).

Refitting of a bladelet core on a small elongated cortical nodule (a) belonging to a flint block from which blades were produced (b; @: P. Bodu, CNRS \& CAD: M. Ballinger, CNRS). 
pour le gisement de Bidart où la réalité de la production lamellaire ne peut pas être démontrée faute d'éléments probants et parce que des pollutions par des pièces lamellaires provenant de niveaux plus récents sont possibles (Bachellerie, 2011, p. 188189).

Le gisement de Quincay serait donc le seul à livrer des «lamelles de grandes dimensions associées à la présence de lamelles retouchées» (Roussel et Soressi, 2014, p. 45)? Le processus de production de lamelles semble calqué sur celui des lames avec un «recul oblique du débitage» (p. 45). Les nucléus à lamelles ne sont pas des formes réduites des nucléus à lames selon M. Roussel, c'est donc bel et bien une production autonome qui livre ce type de supports (Roussel et al., 2016). Plus proche d'Ormesson, la Grotte du Renne à Arcy-sur-Cure a

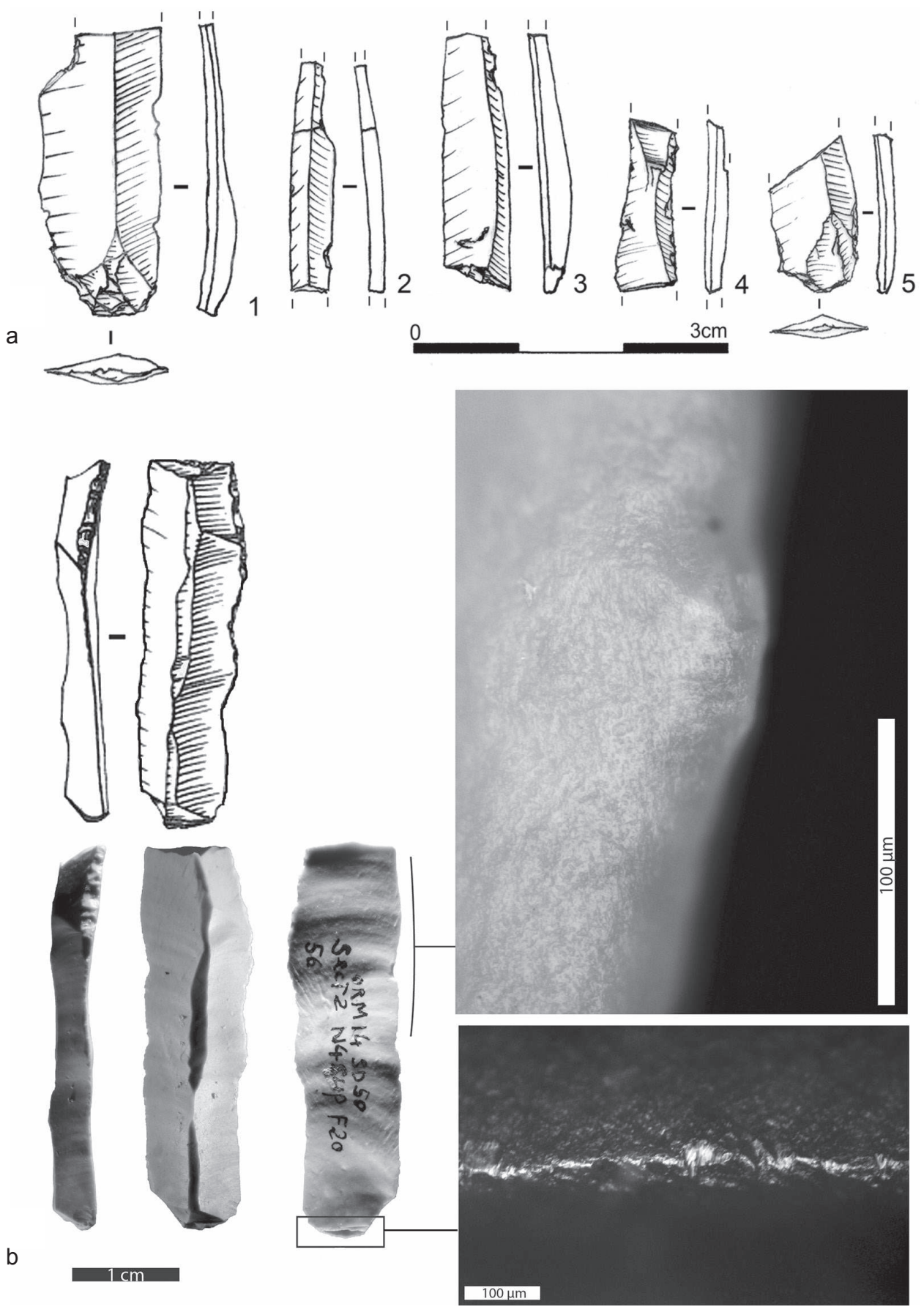

Fig. 28 - a : Lamelles aux bords légèrement abîmés (DAO : M. Ballinger, CNRS et M. Baillet, PACEA); $b:$ La lamelle à bord retouché et à troncature du sondage 50 : clichés de microtraces d'utilisation (DAO: M. Ballinger, CNRS et M. Baillet, PACEA). 
livré une séquence châtelperronienne de référence pour tout le nord de la France (Bodu, 1990; Farizy, 1990; Leroi-Gourhan, 1964). Plusieurs dizaines de milliers d'artefacts taillés en chaille comme en silex ont été classés et étudiés par N. Connet dans le cadre de sa thèse, dont nous rapportons ici les principales conclusions (Connet, 2002). Ces séries châtelperroniennes livrent assez peu d'évidences d'une production lamellaire autonome. Les lamelles semblent être intégrées à la production laminaire sans qu'il y ait véritablement de nucléus à lamelles. Les lamelles des niveaux châtelperroniens de la Grotte du Renne pourraient être, en réalité, des petites lames ou au moins intervenir à l'issue du débitage des lames. Cette constatation va de pair avec le très faible nombre de lamelles retouchées retrouvées dans les niveaux châtelperroniens d'Arcy, car les pièces à retouche abrupte mince décrites par N. Connet sont, le plus souvent, sur supports laminaires de petites dimensions plus que sur de véritables lamelles, observation également menée sur le matériel de Canaule II (Bachellerie, 2011). À Arcy, la couche châtelperronienne supérieure, la couche VIII, a livré seulement trois fragments de lamelles à retouche inverse abrupte et mince, mais rappelons que le niveau VIII est situé sous le niveau VII Proto-Aurignacien où les lamelles Dufour sont particulièrement abondantes. Le risque de migration d'objets de petite taille notamment du Proto-Aurignacien vers les niveaux les plus récents du Châtelperronien est donc très probable. Cependant dans les niveaux châtelperroniens $\mathrm{Xb}$ et $\mathrm{Xc}$, les plus profonds de la Grotte du Renne, les lamelles retouchées sont plus nombreuses et se distinguent morpho-dimensionnellement parlant des lamelles retouchées aurignaciennes. Plus larges pour la plupart que des lamelles retouchées classiques $(1>13 \mathrm{~mm})$, elles sont considérées comme des petites lames plutôt que comme des lamelles (Connet, 2002). À côté de «l'évidence Quincay» (Roussel et al., 2016), la réalité d'une production lamellaire châtelperronienne est néanmoins évidente même si son interaction avec la production laminaire demande a être mieux précisée et son implication dans l'outillage plus amplement argumentée. Le site d'Ormesson peut participer à ce débat. On a ainsi vu précédemment que les lamelles, entières ou fragmentées, étaient très nombreuses dans le niveau châtelperronien d'Ormesson. Elles présentent des dimensions et une régularité qui d'une part les éloignent du gabarit des lames, même si la séparation avec les plus petits modules de lames n'est pas toujours des plus aisées et d'autre part permettent de les considérer comme des produits de première intention et non uniquement des sous-produits du débitage laminaire (tabl. V et tabl. VI). Enfin, et malgré leur faible quantité pour le moment, les lamelles retouchées existent à Ormesson, dans les deux sondages ayant livré du Châtelperronien qui plus est, montrant qu'elles constituent un des objectifs des productions lithiques. Si au sein du sondage 3, les retouches identifiées sur 4 lamelles seulement demeurent assez discrètes (retouches marginales directes ou inverses sur une partie de l'un des bords ou une encoche), dans le sondage 50 l'unique lamelle véritablement retouchée présente une retouche directe, courte et partielle sur la partie distale du bord droit (fig. 28). Le même type de retouche légère reprend partiellement la cassure distale de la lamelle, une sorte de troncature incomplète en somme. Ce n'est pas une retouche épaisse ni abrupte, mais plutôt un léger cadrage, destiné sans doute à la rectification et à l'alignement du bord, mais son intentionnalité ne fait aucun doute. On peut estimer que la troncature distale a le même objectif, à savoir un cadrage. Longue à son abandon de $37 \mathrm{~mm}$ pour $10 \mathrm{~mm}$ de largeur et $4 \mathrm{mmm}$ d'épaisseur, cette lamelle à dos partiel et à légère troncature a été réalisée sur un support aux bords parallèles et relativement réguliers et elle présente une section trapézoïdale. Ses dimensions l'éloignent des dimensions classiques des petites lames, notamment la largeur inférieure à $12 \mathrm{~mm}$ qui ne doit rien à la réduction du support par la retouche. Il s'agit donc bien d'une lamelle à dos tronquée, même si le dos est peu épais et partiel. S'agit-il d'une catégorie d'outil à part entière, mais comment explique-t-on l'unicité de l'objet ou ne pourrait-on pas y voir là l'une des déclinaisons des couteaux châtelperroniens bien qu'il manque la partie distale pointue et alors que les dimensions de l'objet, comme nous venons de le préciser, sont inférieures à celles des plus petites Châtelperrons identifiées à Ormesson? Autrement dit, la lamelle retouchée d'Ormesson ne serait-elle pas une Châtelperron d'appoint au même titre que certaines lames et certains éclats laminaires qui possèdent un bord partiellement retouché12 opposé à un tranchant? Dans ce cas de figure, quel rôle jouent les rares autres lamelles qui portent des retouches discrètes et surtout les centaines de fragments lamellaires non retouchés? En tout état de cause, la lamelle retouchée du sondage 50 n'est pas une Dufour sous type Dufour, comme celles qui ont été identifiées dans les trois niveaux châtelperroniens de Quincay (Roussel, 2011), mais elle s'apparente à ce qui a été observé à Canaule II par exemple (Bachellerie, 2011) et peut-être dans certains autres gisements du sud-ouest.

Les néandertaliens-châtelperroniens d'Ormesson, s'il s'agit bien de cette population, auraient-ils développé seuls et de façon autonome une production lamellaire et les outils afférents, sans avoir été influencés par une «diffusion par stimulus» (Tostevin, 2007; Roussel, 2011) auprès de Proto-Aurignaciens (Roussel et al., 2016)? Le site d'Ormesson ne permet pas encore de répondre à cette question ni de proposer pour le moment d'autres hypothèses. Cependant, dans ce site, le fait que la production lamellaire ne s'éloigne guère de la production laminaire en différents registres (technique, modalités de fabrication, proximité dimensionnelle entre les plus petits couteaux de Châtelperron et les rares lamelles retouchées) témoigne plutôt d'un continuum conceptuel entre les deux productions. Cela fait douter de l'origine Proto-Aurignacienne par diffusion du seul pan lamellaire châtelperronien ou alors c'est l'ensemble du concept de la production lamino-lamellaire des Châtelperroniens qui a été «emprunté» aux Proto-Aurignaciens. Un dernier argument en faveur de la consistance de la production lamellaire, mais dont l'occurrence est cependant faible, est donc l'existence de rares nucléus à lamelles dans les sondages 3 et 50, des nucléus qui n'auraient absolument pas pu donner des lames. Cela concerne les trois nucléus du sondage 3 évoqués précédemment (fig. 27) et le nucléus sur éclat découvert dans le sondage 50 (fig. 26, $\mathrm{n}^{\circ} 3$ ). Une partie des négatifs des produits obtenus sur ces différents nucléus de petite taille sont très clairement des négatifs

12. Retouche intentionnelle ou d'utilisation. 
de lamelles, ce qui témoigne du caractère intentionnel de cette production. Production qui ne cadre pas avec les dimensions des plus petits couteaux de Châtelperron du site, et dont on peut penser qu'elle était vouée à un autre type d'utilisation. Ce type de nucléus notamment sur éclat n'est pas sans rappeler des exemples de la couche 8 de Roc-de-Combe (Pelegrin, 1995, p. 150) ou encore certains des nucléus du site des Tambourets
(Scandiuzzi, 2008, p. 37). D’autres éléments vont dans le sens d'une production de supports allongés fins et étroits sur nucléuséclat, c'est le cas de chutes de burins épaisses qui ne sont autre que de courtes lames à crête ou sous-crête et dont la longueur ne dépasse pas $6 \mathrm{~cm}$. Avec cette dimension initiale, il est évident que les produits obtenus par la suite ne pouvaient guère être que de petites lames et/ou des lamelles.

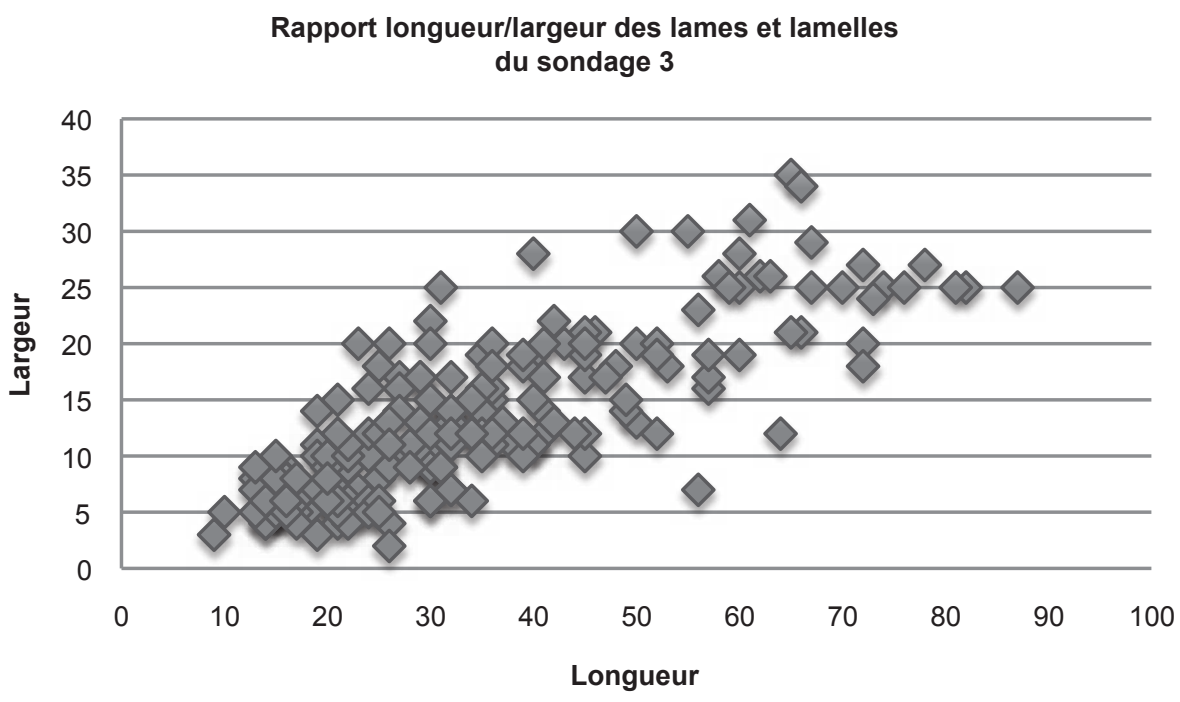

Tabl. V - Rapport longueur/largeur des lames et des lamelles du sondage 3, entières ou presque entières (mesures en mm ; population : 200). Observez les deux populations et le fort regroupement des valeurs entre 3 et $20 \mathrm{~mm}$ de largeur qui caractérise la population des lamelles et des petites lames. La rupture est plus franche avec des lames d'une largeur égale ou supérieure à $25 \mathrm{~mm}$.

Length/width ratio of the blades and bladelets from test-pit 3, intact or almost intact (measured in mm; total number: 200). The distinction between two populations and the cluster of the values between 3 and $20 \mathrm{~mm}$ width characterizing the bladelets and small blades can be observed. The difference with blades having a width equal to or greater than $25 \mathrm{~mm}$ is clear-cut.

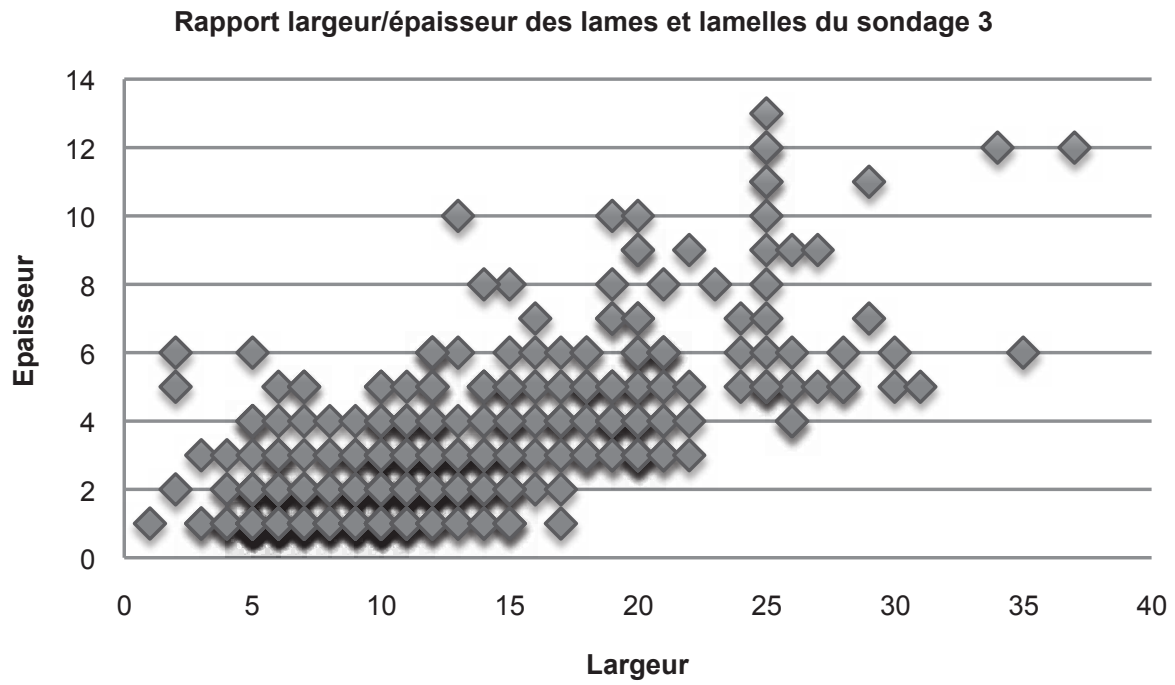

Tabl. VI - Rapport largeurlépaisseur des lames et des lamelles du sondage 3 (mesures en mm; population : 445). Observez les deux populations, celle des lamelles et petites lames distinctes en termes dimensionnels de celle des lames.

Width/thickness ratio of the blades and bladelets from test-pit 3 (measures in mm; total number: 445). They are divided into two groups, the group of bladelets and small blades being distinguishable from the group of blades in terms of dimensions. 
Deux nucléus des sondages 3 et 50 (ensemble 8, nucléus C34.239, fig. 29, $\mathrm{n}^{\circ} 1$; ensemble 13, nucléus E20.104, fig. 29, $\mathrm{n}^{\circ} 2$ et fig. 20), illustrent par ailleurs l'exploitation de petits fragments de blocs qui ne pouvaient absolument pas donner de lames, mais éventuellement des lamelles (longueur d'environ $5 \mathrm{~cm}$ ), mais l'accumulation de cônes incipients et de réfléchissements pour l'un et la faible organisation du débitage ainsi que l'irrégularité des produits obtenus par percussion dure en retrait pour l'autre, plaident plutôt en faveur de débitages mal contrôlés, par des individus au savoir-faire médiocre que d'une réelle volonté de production lamellaire ${ }^{13}$. Un troisième nucléus de plus grande taille (9,5 $\mathrm{cm}$ de longueur) provenant du sondage 3 (ensemble 6, nucléus D34.209), témoigne de la même qualité médiocre du débitage qui se traduit par le détachement de quatre produits d'aspect «laminaire» à la percussion dure à partir d'une crête antérieure plus que sommaire (fig. 29, $\mathrm{n}^{\circ} 3$ ). On retrouve des nucléus exploités à l'identique dans la couche 8 du Roc-de-Combe, même si pour l'exemple illustré (Pelegrin, 1995, p. 149), la mauvaise matière est évoquée pour expliquer la piètre qualité du débitage. M. Roussel présente des débitages aussi mal achevés à Quincay, avec de nombreux négatifs de réfléchissements et un aspect peu lamellaire des derniers enlèvements (Roussel, 2011, fig. 6.26, p. 321 et fig. 6.27, p. 322). À Ormesson, la qualité de la matière n'explique pas tout et il est vraisemblable que l'un, au moins, des tailleurs du groupe ne maîtrisait pas toutes les subtilités du débitage. Il est cependant intéressant de constater que ces exploitations médiocres ont malgré tout pour objectif la production de supports allongés même si l'on ne peut pas véritablement parler de lames ou de lamelles en termes de régularité. Ici comme dans bien d'autres industries du Paléolithique supérieur, les tailleurs médiocres et/ou débutants cherchent à produire ce qui fait l'essence même de la panoplie lithique de leur tradition culturelle, des supports laminaires et/ou lamellaires. Dans le cas du nucléus sur éclat D33.201 à faible production lamellaire (fig. 26, $\mathrm{n}^{\circ} 1$ ), on peut d'ailleurs se demander si le schéma déroulé n'est pas une «imitation» en miniature d'un véritable débitage laminaire sur support de plus grande dimension. Il est évident, même dans ces débitages les moins sophistiqués, que les éclats ne sont pas des produits de première intention, même si certains des éclats les plus épais obtenus lors des phases de mise en forme de grands blocs ont été utilisés comme supports de petits nucléus comme c'est le cas du nucléus D33.201 ou encore de E20.54 (fig. 26). Il n'est pas

13. Ainsi le nucléus E20.104 du sondage 50 ne porte pas moins de 7 ou 8 négatifs de réfléchissements sur la surface prétendument «lamellaire».
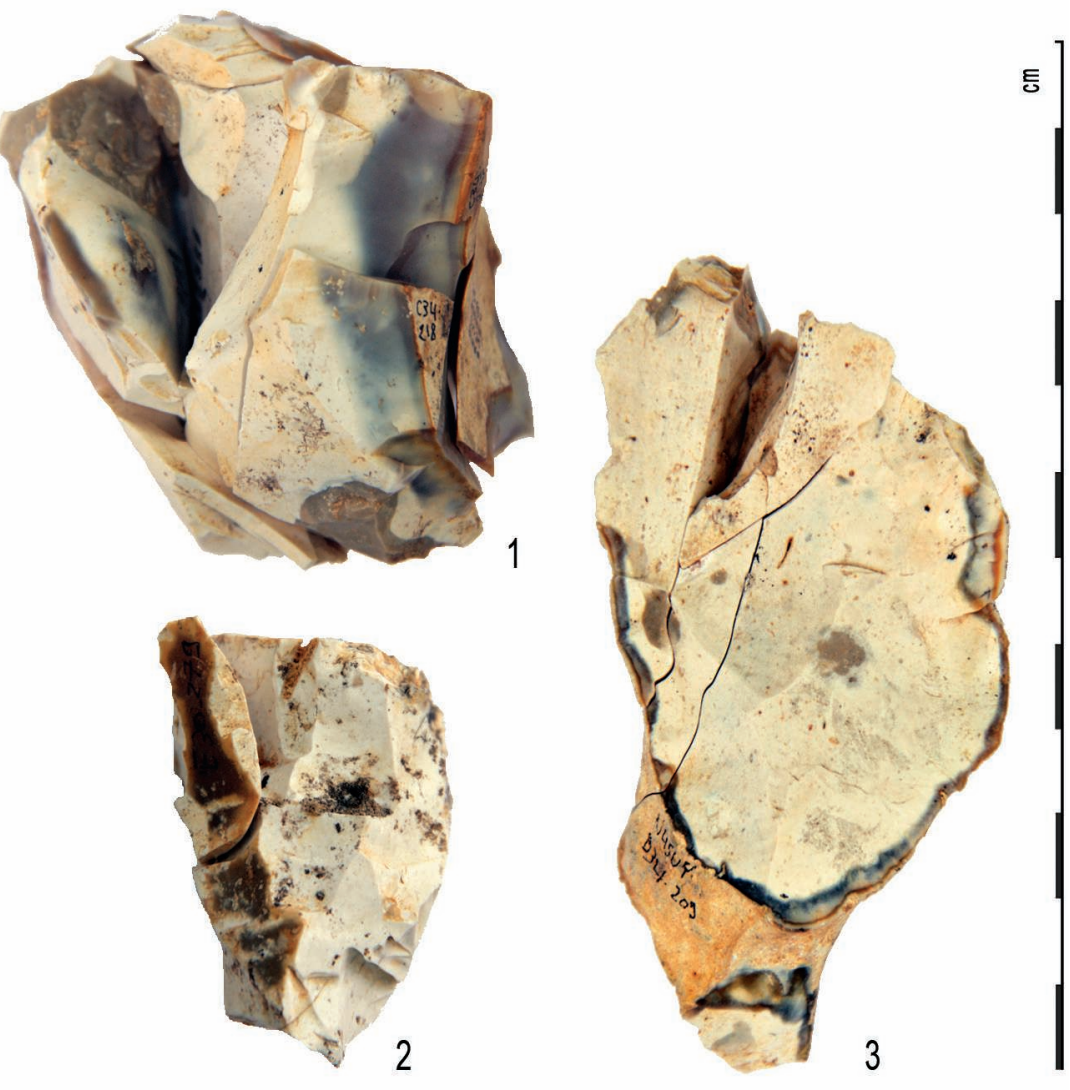

Fig. 29 - Des débitages maladroits (@ : P. Bodu, CNRS).

Clumsy debitages (@: P. Bodu, CNRS).

impossible par ailleurs que le seul emploi de la pierre tendre durant tout le processus ait conduit, au final, à des morphologies de tables lamellaires assez mouvementées, dans la mesure où la pierre même tendre, même utilisée par un bon tailleur, peut provoquer avec une baisse du soin en fin d'exploitation de petits volumes, des négatifs assez irréguliers. Par ailleurs, les nucléus à lames identifiés dans le sondage 3 et dans le sondage 50 ne montrent pas de tentative d'extraction de lamelles en fin d'exploitation. Ainsi les derniers produits utilisables sur ces nucléus mesurent entre 70 et $80 \mathrm{~mm}$ de longueur et s'apparentent en effet plus aux supports des plus petits couteaux de Châtelperron, comme celui identifié dans le sondage 3 (C34.52 et 303) dont la longueur n'excède pas $55 \mathrm{~mm}$ et la largeur $16 \mathrm{~mm}$. La question de l'existence d'un débitage lamellaire intercalé se pose également, à l'exception peut-être du nucléus sur corne corticale évoqué précédemment, mais peu d'indices en témoignent véritablement de sorte qu'il semble exister un réel décalage quantitatif entre le nombre de lamelles ou fragments de lamelles observés dans les deux sondages et les traces de ces enlèvements lamellaires à la fois sur les parties supérieures des lames et sur les nucléus. Étant donné la petite taille de la surface connue pour ce niveau châtelperronien, on peut estimer que l'industrie lithique retrouvée n'est pas entièrement représentative et qu'il manque des éléments plus évidents témoignant des modalités de la production lamellaire. Le débitage des lames à la pierre 
tendre a cependant pour corollaire le détachement d'un certain nombre de lamelles soit de façon délibérée pour améliorer les convexités, soit «accidentellement» comme épiphénomènes lors du détachement d'une lame. Il n'est donc pas exclu que la «production lamellaire»d'Ormesson soit à imputer en partie à ces causes. Par ailleurs les plus petits supports de couteaux de Châtelperron présentent des dimensions qui ne sont pas très éloignées de ce que l'on considère habituellement comme des lamelles, il n'est alors pas impossible que ces dernières représentent les plus petits éléments obtenus lors d'une production de supports pour Châtelperrons. Ainsi si les produits bruts semblent indiquer, par leur quantité, un intérêt évident des châtelperroniens pour les lamelles, l'outillage retouché sur lamelle ne traduit que plus difficilement cet engouement. Il est en effet curieux de constater qu'il existe si peu de lamelles retouchées malgré la forte quantité de lamelles brutes, ici à Ormesson comme dans d'autres gisements évoqués précédemment. L'hypothèse d'une utilisation directe de supports lamellaires bruts a déjà été mise en avant par certains collègues, nous ne pouvons que la reprendre à notre compte au vu des traces d'utilisation que semblent porter certaines des lamelles brutes d'Ormesson, même si la part importante de lamelles brutes peut trouver d'autres explications, ainsi que nous venons de le proposer. Pour cela, il nous faut obtenir une plus grande surface de fouille autour des deux concentrations étudiées, car si l'on se fie à ce que l'on connaît dans des gisements du Paléolithique supérieur, les armatures ou autres lamelles retouchées sont le plus souvent abandonnées dans des emplacements assez spécifiques à proximité d'une structure de combustion. Mais si des indices de combustion ont été clairement identifiés (taches charbonneuses et cendreuses, os et silex brûlés) aucun véritable foyer n'a encore été trouvé dans le niveau châtelperronien.

\section{Les Châtelperrons : des couteaux ou des pointes?}

Dans l'outillage du sondage 3, on compte huit couteaux de Châtelperron (18 \% ; fig. $\left.30, \mathrm{n}^{\circ} 1-8\right)$ alors que le sondage 50 , n'en a livré qu'un fragment proximo-mésial tronqué (fig. 30, $n^{\circ}$ 9) et deux autres fragments possibles. On peut ajouter aux huit objets du sondage 3, cinq fragments d'outils que l'on assimile à la catégorie des Châtelperrons (11\%). Enfin, certains autres objets placés dans des catégories moins formelles, plus anecdotiques (encoches, denticulés, certains produits retouchés) pourraient appartenir à celle des couteaux. Ils ne représentent vraisemblablement que des étapes de fabrication ou d'utilisation et ainsi n'en partagent pas toutes les caractéristiques. Enfin, on suppose que certains burins sont des couteaux altérés lors de l'utilisation, ce qui pose d'ailleurs la question de la fonction de ces objets, mais il peut aussi s'agir de vrais burins réalisés sur des couteaux abandonnés comme tels. Dans le sondage 3, les couteaux représentent donc plus vraisemblablement $30 \%$ de l'outillage si on leur associe les objets et fragments d'objets évoqués précédemment. Le sondage 50 beaucoup moins riche en outils (11) n'a donc donné qu'un couteau de Châtelperron dont il manque la partie distale et deux fragments qui peuvent être assimilés à des parties de couteaux soit un peu plus de $27 \%$ de l'outillage ${ }^{14}$. Dans les sondages 3 comme 50, les supports utilisés sont majoritairement des lames et éclats laminaires (8 cas) et un éclat a été sélectionné (fig. $30, \mathrm{n}^{\circ} 7$ ). Il semble que, dans ce dernier cas, ce soit le tranchant «naturellement» régulier du support qui ait intéressé l'utilisateur même si l'axe de l'outil n'est pas celui du support. Malgré la petite taille du corpus, on observe que les sept supports entiers ou presque montrent des longueurs assez cohérentes comprises entre 53 et $65 \mathrm{~mm}$. Ce sont des longueurs relativement standard pour les couteaux de Châtelperron. Ainsi à Arcy-sur-Cure, les longueurs des pièces entières des couches châtelperroniennes (Xc, Xb, IX) de la grotte du renne (Plisson, Schmider, 1990; Connet, 2002) sont comprises entre 40 et $60 \mathrm{~mm}$. B. Schmider et H. Plisson signalent que ces longueurs sont équivalentes à celles des Châtelperrons des sites de Pairnon-Pair (Lenoir, 1983, p. 192) et de Roc-de-Combe (Pelegrin, 1995, p. 144). Les largeurs des pièces d'Ormesson s'étagent entre $13 \mathrm{~mm}$ pour la plus étroite et $25 \mathrm{~mm}$ pour la pièce la plus large avec une moyenne s'établissant à $18,25 \mathrm{~mm}$ ce qui est très proche de celles obtenues à Arcy. $(18,5 \mathrm{~mm}$ en Xc et $17,85 \mathrm{~mm}$ en IX). Malgré le contexte gîtologique assez différent puisqu'à Ormesson la matière première n'est guère éloignée que de $5 \mathrm{~km}$ environ alors qu'à Arcy, les sources de silex les plus proches sont situées à près de $15 \mathrm{~km}$ et que l'approvisionnement local correspond à de la chaille, il est intéressant de constater que les modules des couteaux sont identiques, à peu de choses près.

Une pièce sort du lot à la fois par une longueur et une largeur plus importantes ( $87 \times 25 \mathrm{~mm}$ ), mais aussi en raison de la morphologie globale de l'objet. La retouche de la partie dorsale est plutôt discrète ne faisant que souligner la rectitude de ce bord droit et l'aménagement de la pointe, ne fait lui aussi qu'accentuer une courbure déjà présente sur la partie distale du support. C'est une Châtelperron bien atypique qui s'apparente à un couteau de «type Audi». Elle partage néanmoins avec les autres plus classiques, la recherche d'une pointe même si celle-ci est déjetée et un bord abattu opposé à une zone tranchante (fig. 30, $\mathrm{n}^{\circ} 1$ ).

Il ne semble pas exister dans ce petit corpus de latéralisation préférentielle du bord abattu donc du tranchant puisque sur les neuf exemplaires considérés, cinq ont un dos abattu à droite et quatre à gauche. Il y a donc de fortes chances pour que cette non-latéralisation, observée sur d'autres gisements (Le Basté, Bidart, Canaule II ; Bachellerie, 2011) soit en fait une adaptation à la morphologie des supports sélectionnés, le dos se plaçant de l'autre côté du bord le plus tranchant et régulier. C'est sans doute cet élément qui guide le choix d'un bord retouché à gauche ou à droite. Le tranchant semble donc essentiel dans la composition de cet outil. Ce qui peut expliquer également que le dos n'a pas toujours ni la même délinéation (parfois rectiligne, parfois courbe), ni le même degré d'investissement par la retouche (parfois épaisse, abrupte et profonde, parfois légère; parfois complète, plus souvent partielle). Le fort état de patine qui affecte la totalité des pièces de ce niveau n'empêche pas l'analyse tracéologique microscopique. Il a en effet été possible

14. Le tri des esquilles nous a permis d'identifier une petite base cassée de Châtelperron (10x10x4 mm). 

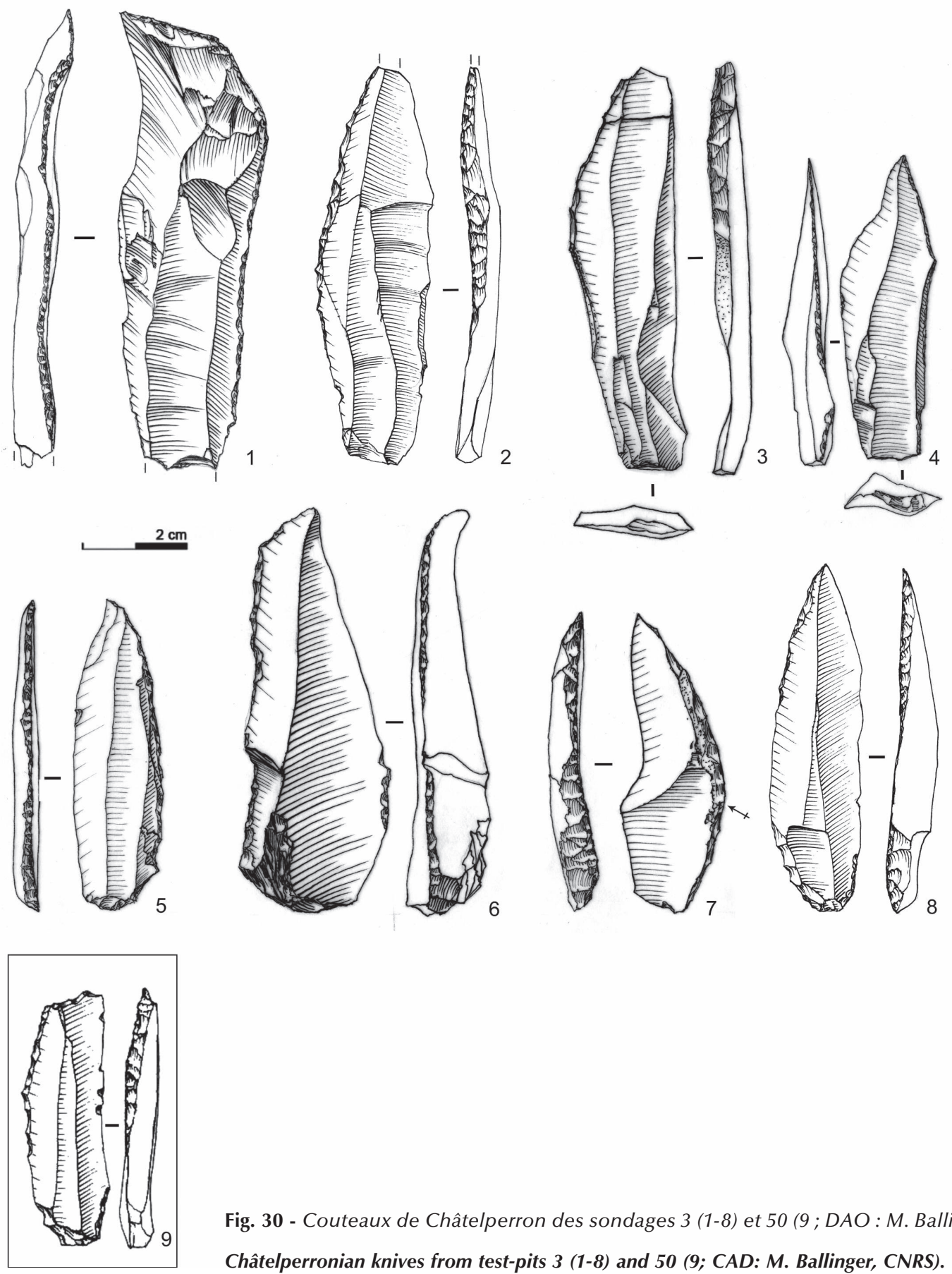

Fig. 30 - Couteaux de Châtelperron des sondages 3 (1-8) et 50 (9; DAO : M. Ballinger, CNRS).

Châtelperronian knives from test-pits 3 (1-8) and 50 (9; CAD: M. Ballinger, CNRS).

d'identifier sur trois Châtelperrons ${ }^{15}$ des esquillements plus ou moins légers des tranchants d'origine anthropique pouvant évoquer une découpe sans brutalité d'une matière organique

15. Les trois provenant du sondage 3 .

16. La lame large élancée assimilable à un couteau de «type Audi» s'avère être intacte. Nous ne pouvons donc trancher la question de son éventuelle proximité typo-morphologique avec les Châtelperrons. mêmes tranchants ${ }^{17}$ (fig. 31). Les couteaux à découper des matières tendres peuvent être mis en parallèle avec une lame à bord abattu (très légèrement par égrisage) qui présente les mêmes stigmates au même endroit. Leur durée de vie a probablement été courte au vu de l'absence à la fois de microémoussé et de poli sur les esquillements. Trois autres Châtelperrons sont manifestement intactes, ce qui peut s'avérer logique pour l'un d'entre eux (E20 n 50 du sondage 50) visiblement abandonné au stade d'ébauche de la retouche. Les observations faites sur le

17. À moins que certains burins soient ces couteaux dont un usage « violent» a conduit à l'enlèvement de chutes. 
matériel d'Ormesson rejoignent celles effectuées par H. Plisson sur la série d'Arcy : «La tendance, entrevue à Arcy, d'un usage de ces pointes comme couteau à matière tendre (carnée?), sera à confirmer par l'examen d'ensembles mieux conservés » (Plisson, Schmider, 1990). De la même façon l'hypothèse est proposée d'une utilisation des pointes en armature de projectile qui auraient subi des impacts et donc des cassures consécutives à ces impacts : «Dans l'état actuel de nos recherches, il paraît difficile de trancher pour telle ou telle fonction, même si l'absence de fractures d'impacts claires ne semble pas plaider ici pour une utilisation en pointe de projectile» (Bachellerie et al. 2007). Soutenant cette hypothèse, l'assemblage de Bidart
(Pyrénées-Atlantiques) révèle des fractures observables sur les pointes qui sont «non diagnostiques d'une utilisation en arme de jet» (Bachellerie, 2011, p. 178). Mais à Brassempouy, dans l'abri Dubalen, la fonction de pointe de projectile est clairement attestée sur certaines Châtelperrons qui portent des «stigmates de choc apical axial» (Bachellerie et al., 2011). Il en est de même au Pays basque dans le site de Labeko Koba où deux pointes ont livré des stigmates dus à une utilisation comme éléments de projectile (Rios-Garaizar, 2008). À Ormesson, aucune trace d'impact n'a été identifiée pour l'instant sur les pointes pourtant fragiles. Cependant, la petite taille de la surface fouillée peut représenter un biais à l'interprétation
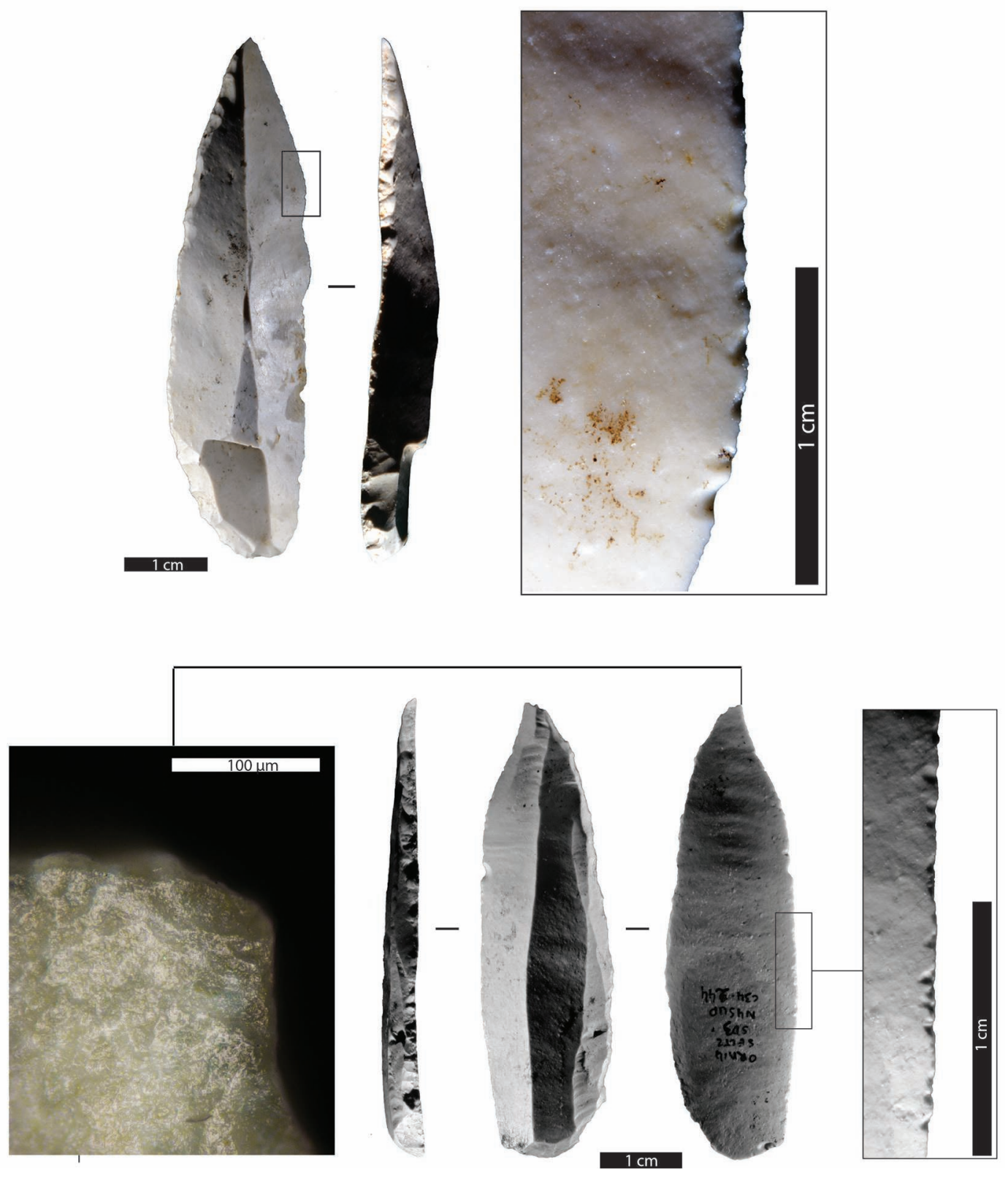

Fig. 31 - Détail de microtraces sur couteaux de Châtelperron (৫) : M. Baillet, PACEA).

Detail of microwears on Châtelperronian knives (৫); M. Baillet, PACEA). 
fonctionnelle de cette catégorie d'objets. Seule une pointe, provenant du sondage 50, montre une cassure distale soulignée par une troncature oblique abrupte assez irrégulière et un tranchant affecté par d'assez nombreux esquillements, des stigmates qui pourraient être liés à une utilisation relativement violente. Mais rien ne prouve qu'il s'agisse d'une utilisation comme armature de projectile. On ne doit pas non plus évacuer l'hypothèse que quelques burins seraient en fait des pointes de Châtelperron cassées à l'usage. Certains d'entre eux présentent en effet des enlèvements burinants dont l'intentionnalité est douteuse. L'abandon de trois burins au sein même de la concentration principale de couteaux de Châtelperron va dans le sens de cette hypothèse (fig. 32).

La mise sur plan des couteaux de Châtelperron et des pièces assimilées du sondage 3 , permet en effet d'identifier un très net regroupement de ces objets en bord ouest de l'amas de silex, où ils sont disséminés sur moins de deux mètres carrés (fig. 32). Il existe là à l'évidence une aire d'activité qui a impliqué préférentiellement des couteaux de Châtelperron, mais également quelques lames ou éclats laminaires portant une retouche plus ou moins discrète d'utilisation, d'affûtage ou de bordage. On est tenté d'y voir une zone de découpe probablement de matière carnée, zone relativement peu dense en autres vestiges. Cette aire de découpe pourrait se prolonger également au nord/nordouest de l'amas, mais de façon plus discrète si l'on en juge par la présence de deux seuls autres couteaux. À cette nette raréfaction des vestiges vers le nord/nord-ouest de la surface fouillée répond donc en marge de l'amas de silex à l'ouest et au sud un espace où différentes activités ont eu lieu. Cet espace se prolonge sans doute plus au sud encore, des fouilles à venir nous le confirmeront. Ces premières observations sur l'organisation au sol des vestiges montrent qu'il existe une cohérence spatiale des objets et donc des activités, ce qui est un atout majeur pour une compréhension palethnographique de l'occupation châtelperronienne.

\section{Des bURINS...}

Les burins sont les seconds outils représentés à Ormesson. Ils sont accompagnés de nombreuses chutes. Dans le sondage 3 ils constituent près de $18 \%$ de l'assemblage ( 8 burins), comme les pointes. Il n'y a pas de véritable burin-outil dans le sondage $50^{18}$. Les 8 burins du sondage 3 ont donné, $a$ minima, 36 chutes. Ils se répartissent en burins d'angle sur cassure (3), burins dièdres d'angle et d'axe sur troncature (2), burin dièdre d'axe (1) et deux burins dont les parties actives sont cassées, mais dont on identifie la nature par la portion distale des négatifs de chutes ou une cassure en $\mathrm{S}$ typique (fig. 33). Deux de ces burins d'angle (un simple, un double) posent la question de l'origine des enlèvements burinants en raison de leur morphologie et de la retouche d'un bord, proche de celle des couteaux (fig. $33, \mathrm{n}^{\circ} 3$ ). Ces derniers auraientils été réutilisés en burins? Une fois le tranchant abîmé par des découpes successives il peut en effet sembler logique de

18. L'unique burin retrouvé est en fait le nucléus à lamelles/burin transversal décrit précédemment. rentabiliser les supports. L'écrasement de certains pans est en effet étonnant et il peut être imputé soit à un manque de soin lors du détachement des dernières chutes, soit à une cassure burinante consécutive à un impact. Nous ne pouvons être affirmatifs sur l'origine de ces stigmates, mais à minima on doit considérer que certains de ces burins ont été des Châtelperrons à un moment donné de leur fonctionnement, ce qui enrichit cette catégorie, la mieux représentée parmi les outils, rappelons-le. C'est un constat également fait pour certains burins du site de Quincay, «aménagés sur des pointes/ couteaux de Châtelperron » (Roussel, 2011, p. 98), donc des reprises de pointes ou couteaux, abandonnés après leur utilisation première. C'est un comportement économique assez courant au Paléolithique supérieur où les lames tranchantes retouchées ou non sont fréquemment réutilisées sous forme d'autres outils (grattoirs, burins, becs) à l'une ou aux deux extrémités. Cela ne doit pas cependant nous faire oublier la présence à Ormesson de vrais burins, dont au moins deux qui, à l'évidence, ne sont pas des Châtelperrons accidentées (fig. $33, \mathrm{n}^{\circ} 1$ et 4 ). L'un de ces burins présente sur une dent des stries profondes dues à un raclage contre une matière dure indéterminée, tandis que le tranchant de son bord gauche montre des esquillements possiblement liés à une découpe de matière dure organique (fig. $33, \mathrm{n}^{\circ} 1$ et fig. $34, \mathrm{n}^{\circ} 1$ ). Deux chutes de burin témoignent des mêmes types de traces, respectivement aux mêmes endroits que celles présentes sur le burin. Une certaine prudence est nécessaire cependant en raison de l'absence d'attributs tracéologiques que l'on serait pourtant en droit d'attendre ici, tels que des microémoussés et/ou des polis. Deux burins, aménagés sur deux fragments d'une même lame à dos originelle (dont on ignore l'origine de la fracture initiale), présentent des esquillements marginaux continus et unifaciaux sur leur pan (fig. 33, n ${ }^{\circ} 2$ et fig. 34, $\mathrm{n}^{\circ}$ 2). Ces derniers peuvent correspondre au raclage bref d'une matière dure indéterminée. Des traces semblables se retrouvent sur une chute de burin. Là encore, l'absence troublante de microémoussé ainsi que de poli d'usage nous incite à la prudence. Un autre burin a été utilisé non pas sur son pan, demeuré intact, mais sur son bord tranchant adjacent, sans que l'on puisse connaître l'ordre de ces deux événements. Il s'agit d'une percussion lancée tranchante sur une matière dure organique indéterminée. Cette utilisation s'est soldée par la fracture mésiale en $" S$ $»$ de la pièce, initiée à partir de la zone active. Un autre burin enfin (sur troncature concave et retouche latérale réalisées au percuteur de pierre) pourrait en réalité être le résultat d'une utilisation en pièce esquillée, sur une matière dure organique indéterminée, à l'aide d'un maillet également en matière dure organique indéterminée. Ces différents objets et leurs déchets de fabrication et d'affûtage sont les ultimes témoins d'une activité qui nous échappe pour l'instant, travail de l'os, du bois, d'autres matériaux? La rareté et l'état de conservation médiocre des restes fauniques des sondages 3 comme 50 ne nous permettent pas d'aller plus loin dans l'attribution fonctionnelle de ces burins, mais l'observation préliminaire de stries et pans de raclage sur certains des fragments osseux est un résultat prometteur. À Arcy, dans la Grotte du Renne, les burins châtelperroniens $(5,9 \%$ de l'outillage soit 
231 pièces) ont au moins une raison d'exister, le travail des matières dures animales qui a donné lieu à la production de poinçons, épingles, lissoirs et éléments de parures (Baffier et Julien, 1990 ; d'Errico et al., 2001).

Très peu présents sur le site de Canaule II (Bachellerie, 2011), de l'ordre de 2 à $3 \%$ de l'outillage, les burins sont peu nombreux également au Basté $(4,2 \%)$ et dans ces deux gisements F. Bachellerie n'exclut pas que certains d'entre eux aient été des nucléus «laminaires», mais sans doute doit-on lire nucléus à lamelles. À Quincay, ils sont plus nombreux (9-10\% quel que soit le niveau) et il est intéressant de noter que la catégorie dominante est la moins sophistiquée, à savoir les burins sur cassure, ce qui rappelle en effet les observations faites sur les burins d'Ormesson (Roussel, 2011). À l'évidence, la faible représentation des burins en général dans les sites châtelperroniens est résumée à travers les termes suivants : «quelques burins simples» (Pelegrin et Soressi, 2007) pour caractériser la présence des burins châtelperroniens.

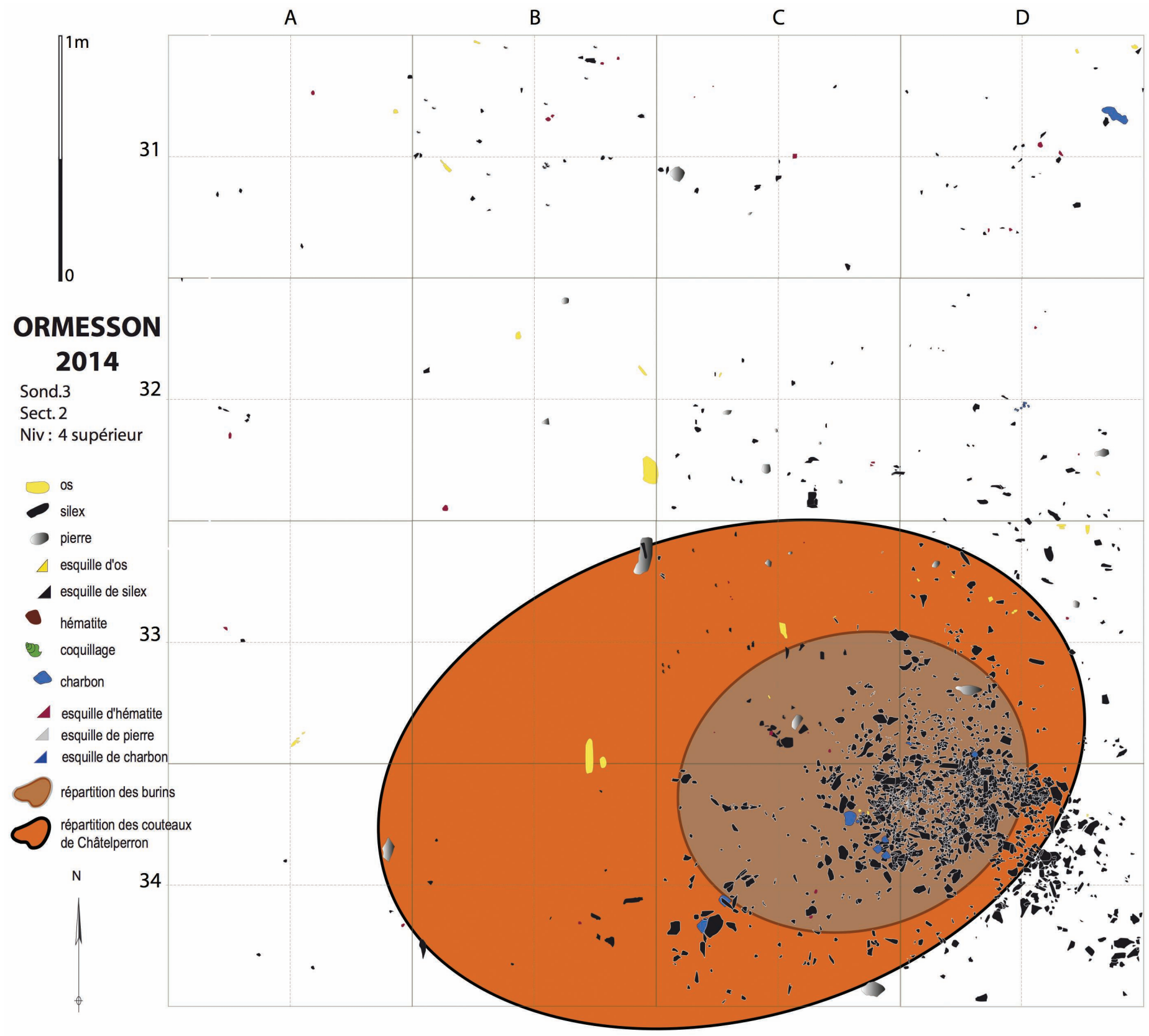

Fig. 32 - Plan de répartition des couteaux de Châtelperron (ovale orange) et des burins (ovale marron) du sondage 3 (DAO : M. Ballinger, CNRS).

Distribution map of the Châtelperron knives (orange oval) and burins (brown oval) in test-pit 3 (CAD: M. Ballinger, CNRS). 


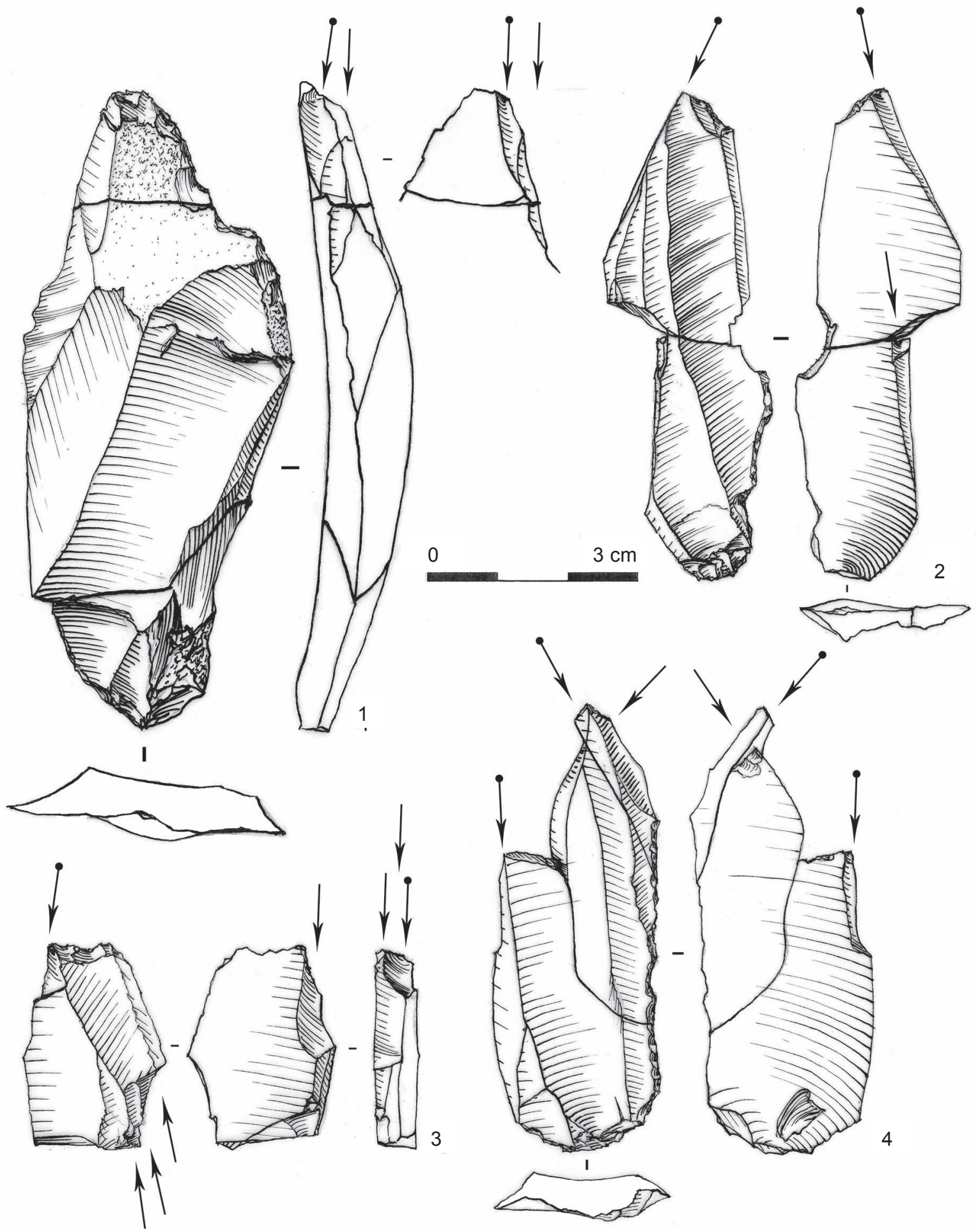

Fig. 33 - Burins du sondage 3 (DAO : M. Ballinger, CNRS). Burins from test-pit 3 (CAD: M. Ballinger, CNRS). 


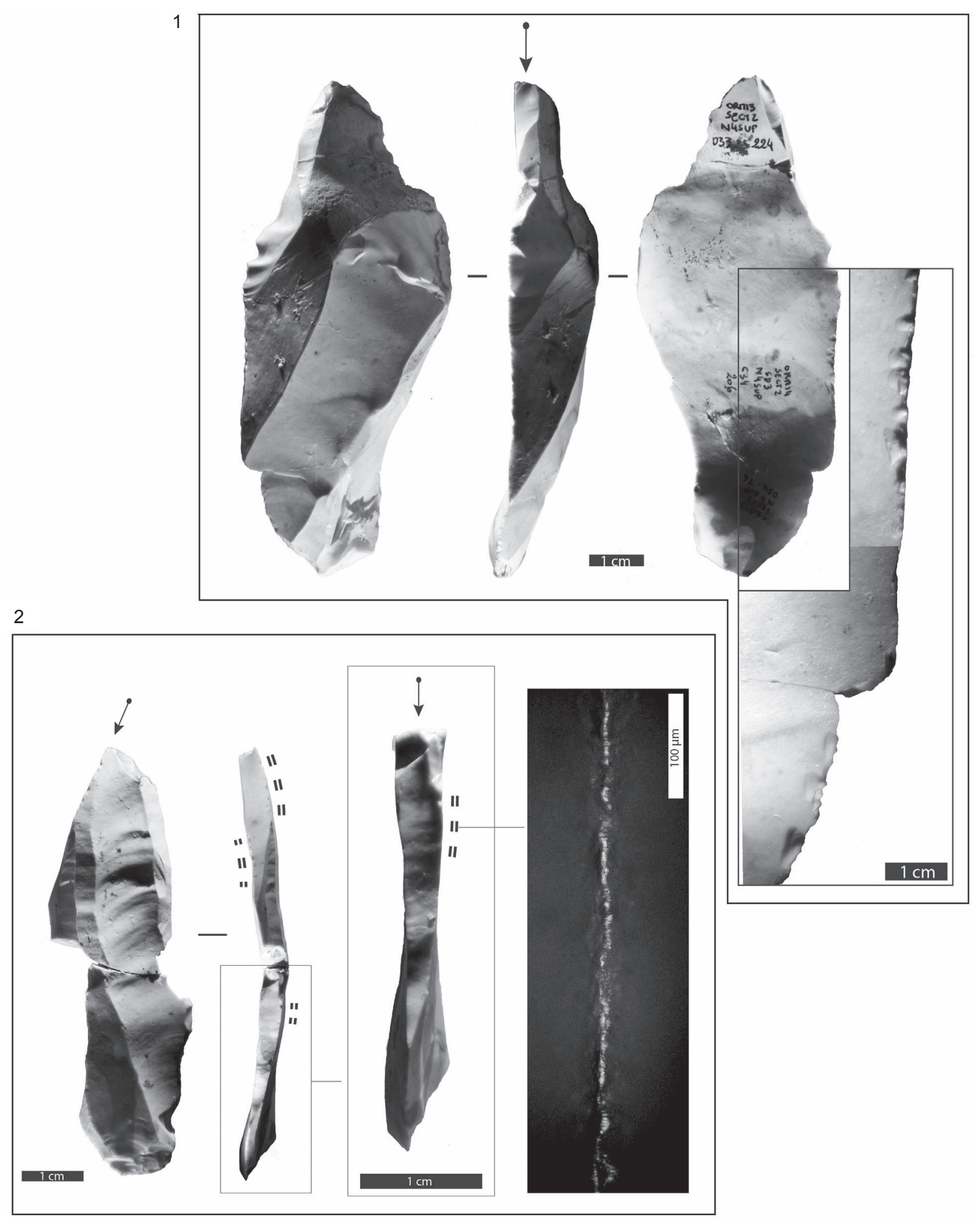

Fig. 34 - Détail de microtraces sur deux burins (C) : M. Baillet, UMR 5199 PACEA).

Detail of microwears on two burins (C): M. Baillet, UMR 5199 PACEA). 


\section{Des GRATtOIRS ABSENTS... DE LA ZONE FOUILlÉE}

L'absence de grattoirs à Ormesson est singulière alors que dans de nombreux gisements châtelperroniens, il s'agit de la catégorie la mieux représentée ou arrivant en seconde position après les couteaux. L'importance numérique de ces outils, qui pourtant ont moins attiré l'attention des préhistoriens que les fameuses pièces à dos, mérite d'être soulignée (Baillet, 2010; Baillet et al., 2014). Ils sont ainsi plus de $16 \%$ au Basté et 27,6 \% à Canaule II (Bachellerie, 2011), ils représentent 19 à $20 \%$ à Quincay (Roussel et Soressi, 2014). Aux Tambourets, les grattoirs épais forment $52 \%$ de l'outillage (Scandiuzzi, 2008), à la Côte N.III, il est fait état de 22 grattoirs pour 158 outils soit près de $14 \%$ de l'outillage (Pelegrin, 1995). Plus près d'Ormesson, les grattoirs sont nombreux dans le matériel lithique des couches châtelperroniennes d'Arcy-surCure (près de 200 objets), mais ils ne représentent guère que $5 \%$ de l'ensemble de l'outillage. Leur absence totale à Ormesson, dans le niveau châtelperronien peut être due à différentes causes : petite taille de la surface fouillée, surface non représentative de l'ensemble des activités qui se sont déroulées sur place, absence à Ormesson de l'activité qui aurait impliqué des grattoirs (le travail des peaux en l'occurrence), utilisation d'autres outils pour le travail des peaux à l'instar de manches coudés en bois de cervidé ou d'outils en os qu'utilisaient ou utilisent encore certaines populations amérindiennes

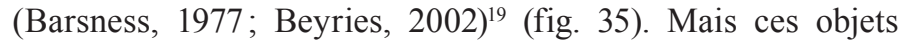
restent à trouver à Ormesson... Les grattoirs, dans d'autres gisements du Paléolithique supérieur, sont souvent retrouvés à quelques mètres de la zone centrale d'activité structurée autour d'une aire de combustion. Dans l'aire ouverte et restreinte des sondages 3 et 50 , on est sans doute soit trop proche de cette zone centrale (sondage 3), soit dans une aire affectée à d'autres activités comme en témoigne la forte présence de lames non retouchées dans le sondage 50 . Alors que la fonction de grattoir dans le gisement de Canaule II par exemple a été reconnue comme d'importance, nécessitant un savoir-faire, une gestion des supports, et qu'elle émane d'un véritable artisanat (Baillet, 2010; Baillet et al., 2014), il nous semble que l'absence de ces outils à Ormesson n'est pour l'instant que circonstancielle.

\section{LAMES ET AUTRES PRODUITS À RETOUCHES DISCRÈTES}

Dans le sondage 3, c'est une catégorie forte de 7 objets, qu'il s'agisse de lames portant des retouches généralement discrètes ( 5 ; fig. $36, \mathrm{n}^{\circ} 2$ et 4 ) comme de lames aux bords non retouchés, mais qui peuvent avoir été utilisées ( 2 ; fig. $36, \mathrm{n}^{\circ} 1$ et 3 ). Quatre éclats laminaires, 1 éclat et 3 lamelles complètent cette catégorie. Cet ensemble hétéroclite en termes d'importance et d'incidence de la retouche affiche cependant une certaine cohérence spatiale puisque les lames ne se retrouvent que dans

19. La même question concernant l'absence ou le faible nombre de grattoirs est posée pour le locus gravettien puisqu'à cet endroit où les restes de huit bisons ont été découverts, il existe très peu de grattoirs, en tout cas pas en nombre suffisant pour avoir permis le traitement de huit peaux. deux mètres carrés du sondage 3, C34 et D34. Il s'agit à la fois d'objets se trouvant dans l'amas de silex, mais également en marge de ce dernier dans un espace où d'autres types d'outils sont présents notamment des couteaux de Châtelperron. Si l'on ne peut mésestimer l'importance des retouches spontanées ou intervenues accidentellement après le débitage au sein de cette catégorie en particulier en ce qui concerne les produits qui se trouvent dans l'amas, il existe indubitablement un lot de vraies lames retouchées dont une étude tracéologique en cours pourra préciser, peut-être, si ce n'est la fonction au moins la qualité de la retouche et donc son origine.

\section{LES RESTES FAUNIQUES}

Le Châtelperronien est une période assez mal documentée du point de vue des communautés d'herbivores. L'identification des faunes est délicate en raison de la rareté des gisements ayant bénéficié de la préservation du matériel osseux. Ceux-ci sont principalement localisés dans le Sud-Ouest (Armand et al., 2004; Discamps et al., 2011; Discamps et al., 2014; Grayson et Delpech, 2003; Grayson et Delpech, 2008; Morin, 2006; Soulier, 2013) et uniquement en contextes de grottes ou d'abris multistratifiés. La plupart d'entre eux ont subi des perturbations post-dépositionnelles importantes, compliquant l'étude des industries, mais également des restes fauniques. Une exception notable est le site de Roc-de-Combe dont la récente approche taphonomique et archéozoologique a permis de dégager tout le potentiel informatif du niveau châtelperronien du point de vue de l'acquisition et du traitement des carcasses (Soulier, 2013). Pour les autres, les problèmes stratigraphiques ainsi que la fréquentation des carnivores, et en particulier l'hyène, semblent avoir considérablement impacté ces niveaux (Letourneux, 2003; Soressi et Roussel, 2008). La complexité taphonomique concerne également les rares gisements situés plus au nord. Au sein de la grotte du Renne à Arcy-sur-Cure, des occupations d'hyènes ou d'ours sont intercalées entre les occupations humaines (Enloe, 2012). F. David mentionne néanmoins pour la couche $\mathrm{X}$ châtelperronienne une faune bien conservée (NRT $=1060)$, mais très fragmentée. Elle est composée majoritairement de restes de rennes $(50,46 \%$ du NRT), de mammouths $(19,27 \%)$ et de chevaux $(18,35 \%)$ ainsi que de quelques vestiges osseux de bovinés, d'ours, de loups et de lièvres (David et al., 2001).

À Ormesson, bien que les restes osseux soient très mal conservés en raison du contexte gréseux et sableux, quelques vestiges nous sont parvenus à la faveur d'un léger concrétionnement qui les a préservés. L'unique reste déterminable à ce jour est une portion distale de tibia de boviné (fig. 37) sur laquelle nous avons pu mettre en évidence les seules traces de carnivores observables à ce jour à Ormesson (chewing sensu Binford, 1981). L'insertion du muscle fléchisseur du doigt, présente chez Bos sur cet os, est absente sur cette pièce, ce qui est au contraire caractéristique du genre Bison (Olsen, 1960). Nous avons décompté par ailleurs deux fragments de diaphyse d'un ongulé de grande taille (cheval ou boviné) ainsi que 235 fragments osseux indéterminés mesurant moins de $20 \mathrm{~mm}$. Parmi ces restes, 175 sont brûlés soit près de $75 \%$ de l'assemblage. Une 


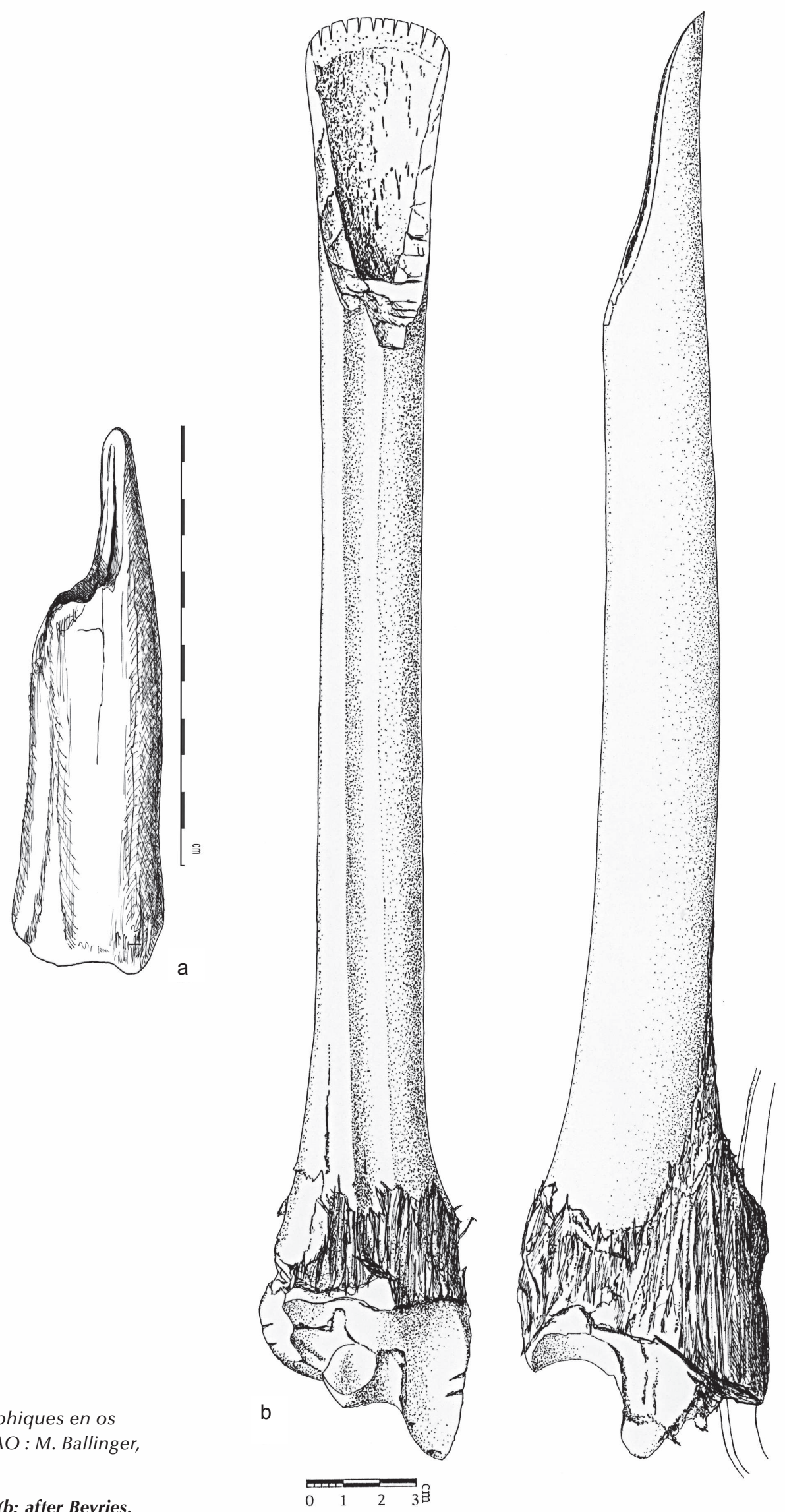

Fig. 35 - Grattoirs ethnographiques en os (b: d'après Beyries, 2002,DAO : M. Ballinger, CNRS).

Ethnographic bone scrapers (b: after Beyries, 2002, CAD: M. Ballinger, CNRS). 

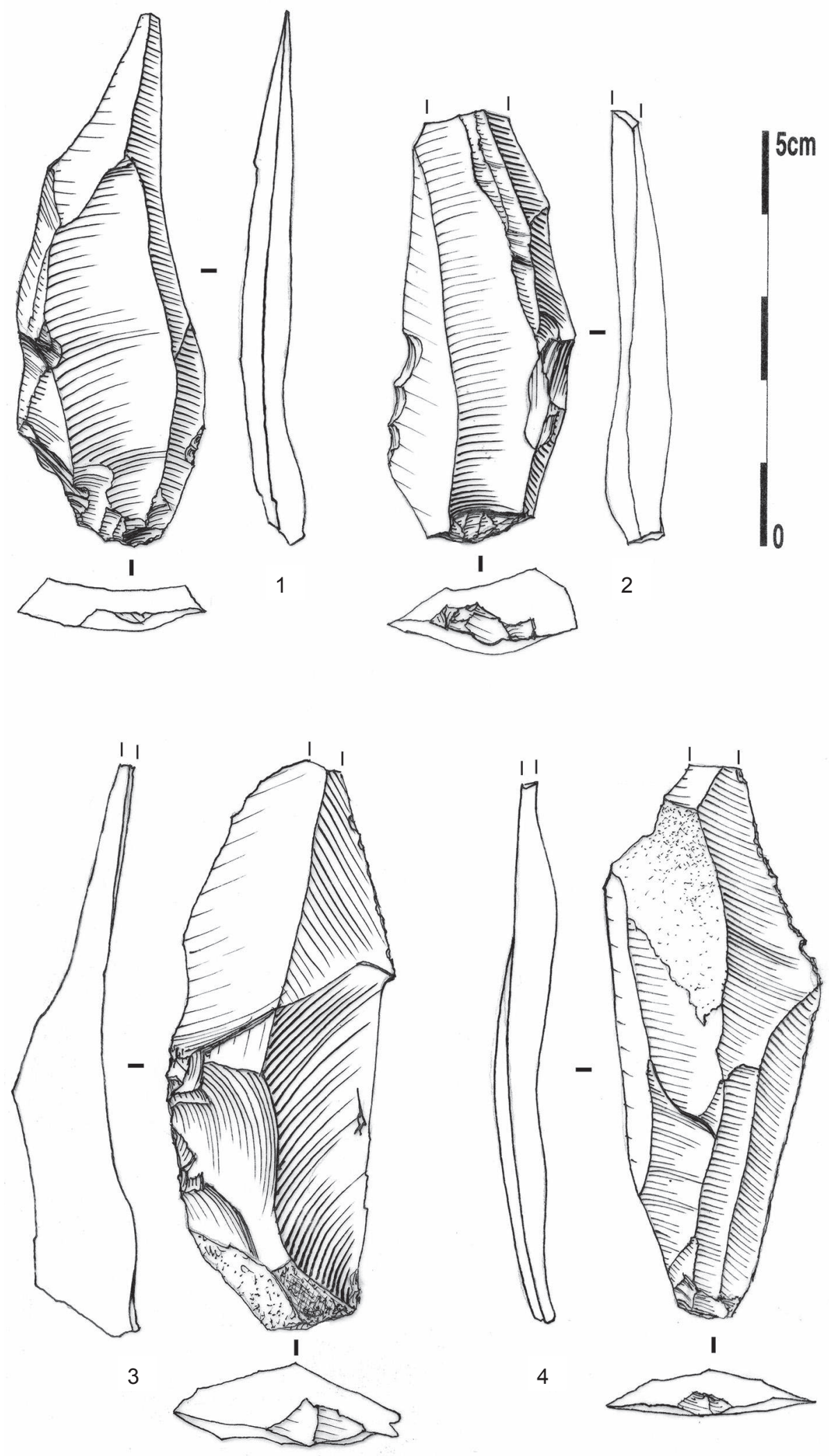

Fig. 36 - Lames à retouches discrètes (DAO : M. Ballinger, CNRS). Blades with limited retouch (CAD: M. Ballinger, CNRS). 
conservation différentielle liée à la chauffe des ossements semble vraisemblable et s'expliquerait par une très mauvaise préservation de la fraction organique du matériel (Gilchrist et Mytum, 1986). Au sein de ce matériel brûlé, des stries de boucherie ont été observées sur trois fragments et du raclage sur deux autres restes (fig. 38), ce qui permet au moins d'attester qu'un traitement des carcasses a été pratiqué sur place avant que les os ne soient exposés à une source de chaleur. Une utilisation des os comme combustible est envisageable bien qu'elle ne soit pas encore démontrable en l'état actuel des données. Elle est en tout cas proposée pour d'autres gisements châtelperroniens (Patou-Mathis, 2005; Soressi et Roussel, 2008).

On peut d'ores et déjà souligner que la présence du bison dans le spectre faunique du niveau châtelperronien d'Ormesson n'est pas incompatible avec les données environnementales du sud-ouest où ce taxon est également renseigné (Morin, 2006; Patou-Mathis, 2005; Soulier, 2013), mais surtout qu'il s'agit d'une récurrence très intéressante par rapport au niveau gravettien où le bison est aussi l'espèce la plus représentée en termes de restes osseux (Lacarrière et al., 2015).
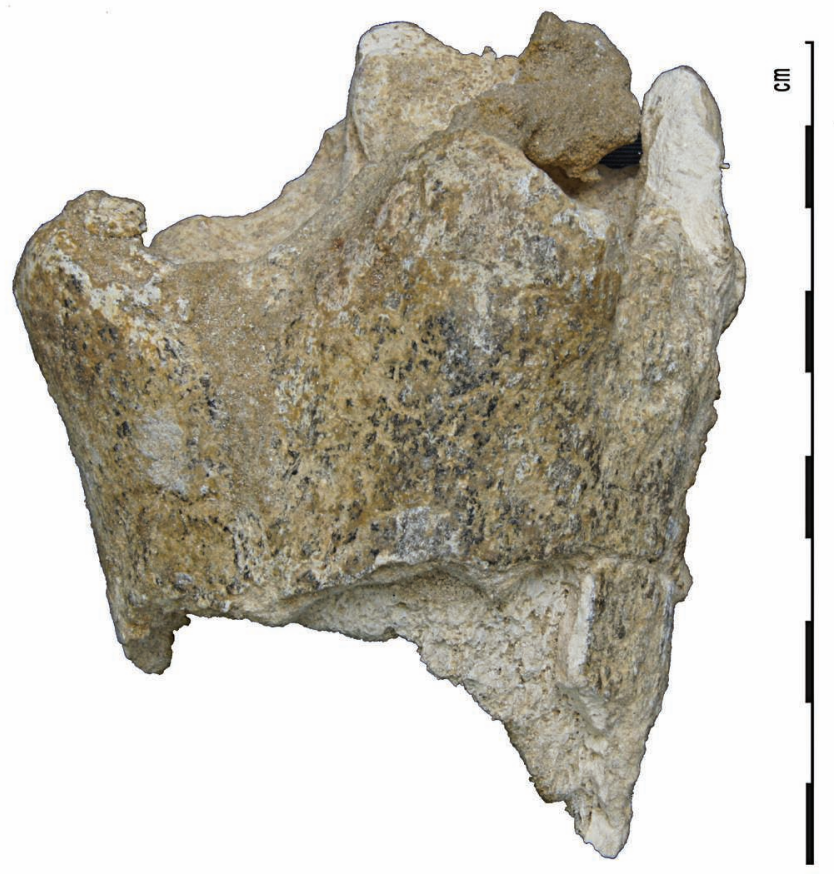

Fig. 37 - Partie distale d'un tibia de bison découvert dans le sondage 3 (C): P. Bodu, CNRS).

Distal part of a bison tibia from test-pit 3 (@: P. Bodu, CNRS).

Fig. 38 - Stries sur deux fragments d'os découverts dans le sondage $3 . n^{\circ} 1: \operatorname{dim}=10 \times 07 \times 05 \mathrm{~mm} ; n^{\circ} 2: \operatorname{dim}=20 \times 08 \times 07 \mathrm{~mm}$. (C): C. Guéret, Arscan).

Striations on two bones from test-pit $3 . n^{\circ} 1$ : size $=10 \times 07 \times 05 \mathrm{~mm}$; $n^{\circ}$ 2: size $=20 \times 08 \times 07 \mathrm{~mm}$ (С: C. Guéret, Arscan).

\section{RÉSULTATS DE L'ANALYSE ANTHRACOLOGIQUE}

Les charbons de bois du niveau Châtelperronien ne sont associés à aucune structure de combustion visible à la fouille. Ils proviennent des carrés C33, C34, D33, D34 du sondage 3 et sont présents en plus forte densité dans le carré C34 $(15 \mathrm{ch} / 10 \mathrm{~g}$ de sédiment) où une tache cendreuse et charbonneuse occupe près d'un demi-mètre carré au sud d'un amas de rejet lithique. $\mathrm{Au}$ total, 456 charbons d'une taille supérieure à 500 microns et 20 microfragments d'os brûlés ont été décomptés, mais seuls 21 fragments, de taille supérieure à $1 \mathrm{~mm}$ ont pu être identifiés (la limite d'identification taxonomique se situant autour de cette classe de taille). L'assemblage extrêmement mal conservé est composé de 13 fragments de pin sylvestre (Pinus sylvestris, Pinus nigra subsp salzmannii, Pinus mugo ou Pinus uncinata (les différentes espèces de pin n'ont pas été discriminées), 1 fragment de genévrier (Juniperus sp.), 6 fragments de gymnosperme et 1 fragment d'angiosperme non identifiables. Si les résidus de combustion sont bien représentés et attestent de l'usage du feu pendant l'occupation châtelperronienne, la petite taille des charbons réduit en revanche les possibilités d'identification spécifique. L'état de conservation du matériel
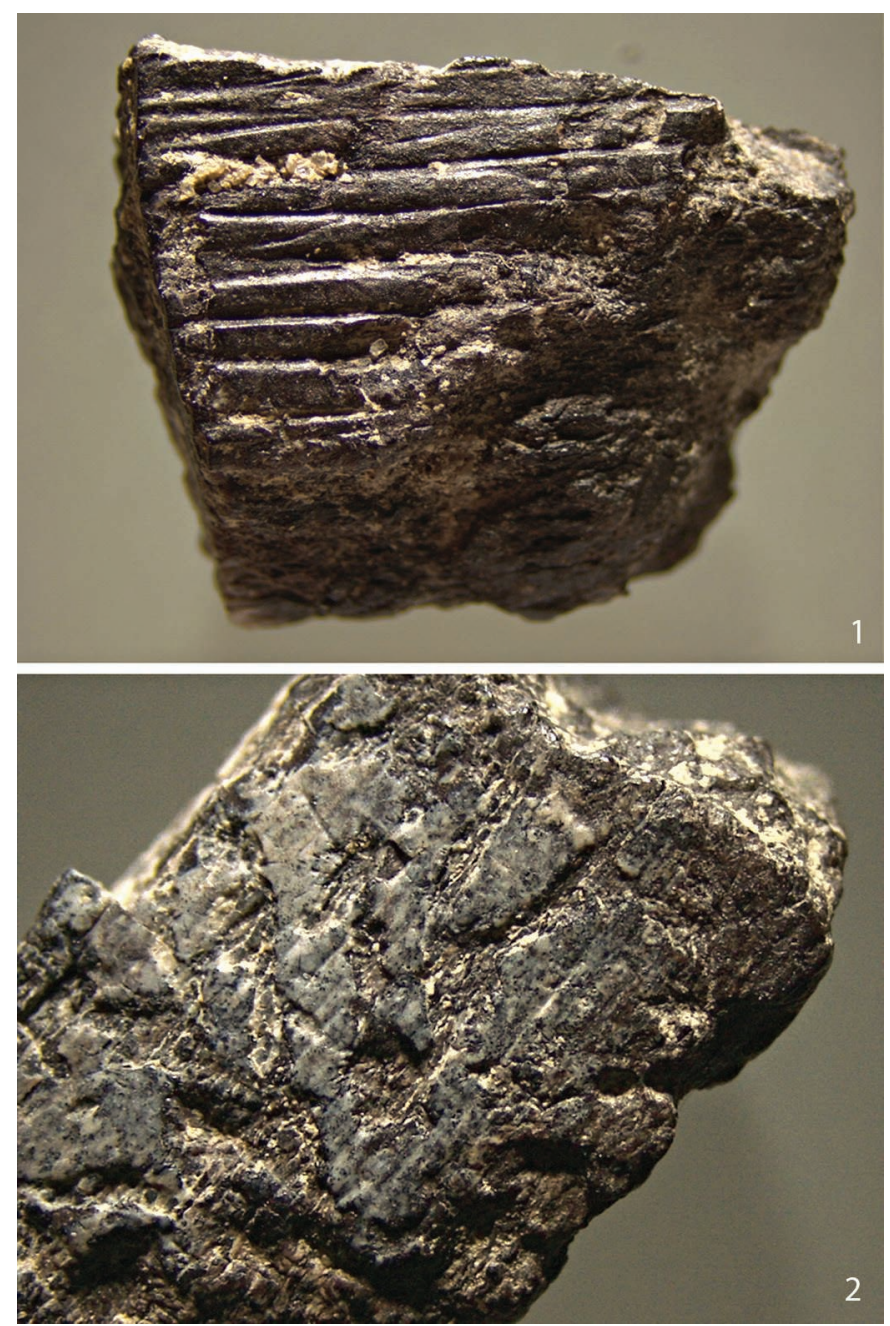
ligneux constitue donc une sérieuse limite de l'étude. Cette intense fragmentation témoigne d'une importante activité postdépositionnelle et n'exclut pas l'hypothèse d'une redistribution sin-sédimentaire ou d'une position secondaire au sein des dépôts pléistocènes, mais cette hypothèse ne s'étend pas à un potentiel mélange entre des niveaux séparés par près de $20 \mathrm{~cm}$ de sable. Le faible effectif exclut toute possibilité d'interprétation quantitative du dépôt. Qualitativement, l'association du pin et du genévrier est typique des dépôts pléistocènes du stade isotopique 4 et du début du stade 3. Elle évoque sans équivoque la flore froide et sèche du dernier glaciaire, caractérisée par une faible diversité spécifique et l'existence d'un paysage relativement ouvert. L'assemblage se distingue peu du niveau moustérien sous-jacent, mais est bien différencié du niveau solutréen où la présence de bouleau (Betula sp.) traduit l'existence d'un milieu plus forestier et plus humide. L'hypothèse d'une sélection du pin, taxon majoritaire, aux dépens d'autres taxons semble difficile à justifier; l'assemblage traduit d'abord une réalité écologiquement cohérente au regard du contexte bioclimatique et des résultats d'études contemporaines. Néanmoins, l'élagage naturel important de cette essence permet un accès rapide à la ressource ligneuse. Dans un contexte de forte mobilité, le bois mort issu de l'élagage naturel, déjà sec et «coupé», est parfaitement adapté au mode de vie des groupes qui voient dans cette ressource facilement accessible un moyen d'approvisionnement rapide et efficace.

\section{QUELQUES INFORMATIONS SUR LES MATIÈRES COLORANTES}

À l'heure actuelle, des matières colorantes ont été mises au jour dans 17 sites ou niveaux châtelperroniens, mais seulement 8 d'entre eux disposent d'une documentation ou d'un contexte sédimentaire suffisamment précis et fiable pour les associer aux restes d'occupation châtelperroniens (Dayet et al., 2014 ; Salomon, 2009; Salomon, 2017 à paraître; fig. 39). Les matières colorantes châtelperroniennes se présentent sous forme de blocs bruts, fragments, blocs facettés par des actions anthropiques volontaires, résidus (rarement mentionnés) et imprégnations dans les sédiments. Sur les six sites châtelperroniens de plein air, quatre en renfermaient. En revanche, bien que les assemblages de matières colorantes soient plus riches en grottes et abris, rares sont les sites dans ces contextes qui en ont livré. Cette situation s'explique probablement par l'ancienneté des fouilles au cours desquelles peu de soin a été accordé à l'enregistrement de ce type d'artefact ou de coloration du sol.

Moins de $100 \mathrm{~g}$ de matières colorantes (soit 537 éléments décomptés) ont été découverts dans le niveau châtelperronien d'Ormesson, principalement sous la forme de petites esquilles de moins de $1,5 \mathrm{~cm}$ de long, dont 28 présentent des traces d'usure résultant d'activités anthropiques volontaires. Le sondage 3 révèle un semis irrégulier de petits fragments de matières colorantes réunissant l'essentiel de l'assemblage (516 pièces, dont 27 avec des traces d'usure). Le sondage 50, quant à lui, compte des pièces éparses, principalement des esquilles sans trace d'usure et, au sommet du niveau châtelperronien, un objet facetté. Toutes les matières colorantes, sauf une, sont des fragments de concrétions yprésiennes, c'est-à-dire exactement la même matière première que celle qui a été sélectionnée et exploitée par les moustériens du niveau 4. Il s'agit en effet de fragments anguleux de concrétions ferrugineuses constituées de couches concentriques d'hématite et de goethite (Bodu et al., 2014 b; Mathis et al., 2014). Dans le sondage 3, les 27 fragments présentent des traces d'usure telles que des surfaces planes régularisées, striées et/ou lustrées par abrasion, raclage et concassage. Les traces d'usure ont les mêmes caractéristiques que celles qui ont été observées et mesurées dans la collection issue du Moustérien sous-jacent : abrasion sur au moins trois outils en roche grenue de granulométrie différente, raclage avec un outil en silex, concassage après raclage et abrasion. Le seul vestige qui se distingue, par sa lithologie, des concrétions prisées par les moustériens a été découvert au sommet de la couche châtelperronienne dans le sondage 50 où aucun niveau moustérien n'est identifié pour l'instant. Il s'agit d'une concrétion facettée riche en hématite et faite d'une alternance de couches plus ou moins indurées d'hématite et d'un lit de quartz subanguleux (fig. 40). Les caractéristiques lithologiques de cet objet en font une matière première peu compatible avec les ressources que nous avons déjà documentées (Mathis et al., 2014) et donc différente des autres matières colorantes de l'assemblage châtelperronien et de l'assemblage moustérien. Les traces d'usure qui couvrent cet objet contrastent aussi avec les stigmates d'exploitation des concrétions yprésiennes mises au jour dans le reste du niveau châtelperronien et dans le niveau moustérien. En particulier, une première réduction du volume par concassage - marquée par des négatifs d'impact opposés résultant de la percussion du bloc avec un outil mobile sur une surface dure immobile - a été suivie d'opérations d'abrasion sur des surfaces rugueuses. Les arêtes des cassures ont été légèrement émoussées par ces dernières actions. À l'inverse, les concrétions yprésiennes mises au jour dans le Moustérien et le Châtelperronien ont été abrasées dans un premier temps, puis fragmentées dans un second temps. Dans le sondage 3, les niveaux moustérien et châtelperronien sont séparés par une quinzaine de centimètres d'épaisseur de sable. De ce fait, des déplacements verticaux d'esquilles yprésiennes sont probables. Ils pourront, le cas échéant, être évalués et caractérisés lors de la fouille de l'inter-couche châtelperronien-moustérien.

\section{L'ANALYSE SPATIALE : DES RÉSULTATS PRÉLIMI- NAIRES, MAIS PROMETTEURS}

L'analyse spatiale du niveau châtelperronien d'Ormesson ne peut guère concerner que le sondage 3 , le sondage 50 ayant une surface ouverte de trop petite taille. Dans le sondage 3 c'est l'amas de silex situé en C-D/33-34 qui structure véritablement l'espace associé à une aire charbonneuse et cendreuse dont il est bien difficile de définir la fonction. La nature de l'amas de silex fort de plus d'un millier de pièces est tout aussi délicate à affirmer, cependant l'incomplétude des différents ensembles taillés remontés plaide plutôt en faveur d'un amas de rejet. Les vides dans les remontages, qui ne correspondent pas uniquement à des produits de première intention, s'expliqueraient ainsi par le fait que tout ou partie des objets manquants sont restés à l'emplacement du ou des vrais postes de taille que nous 


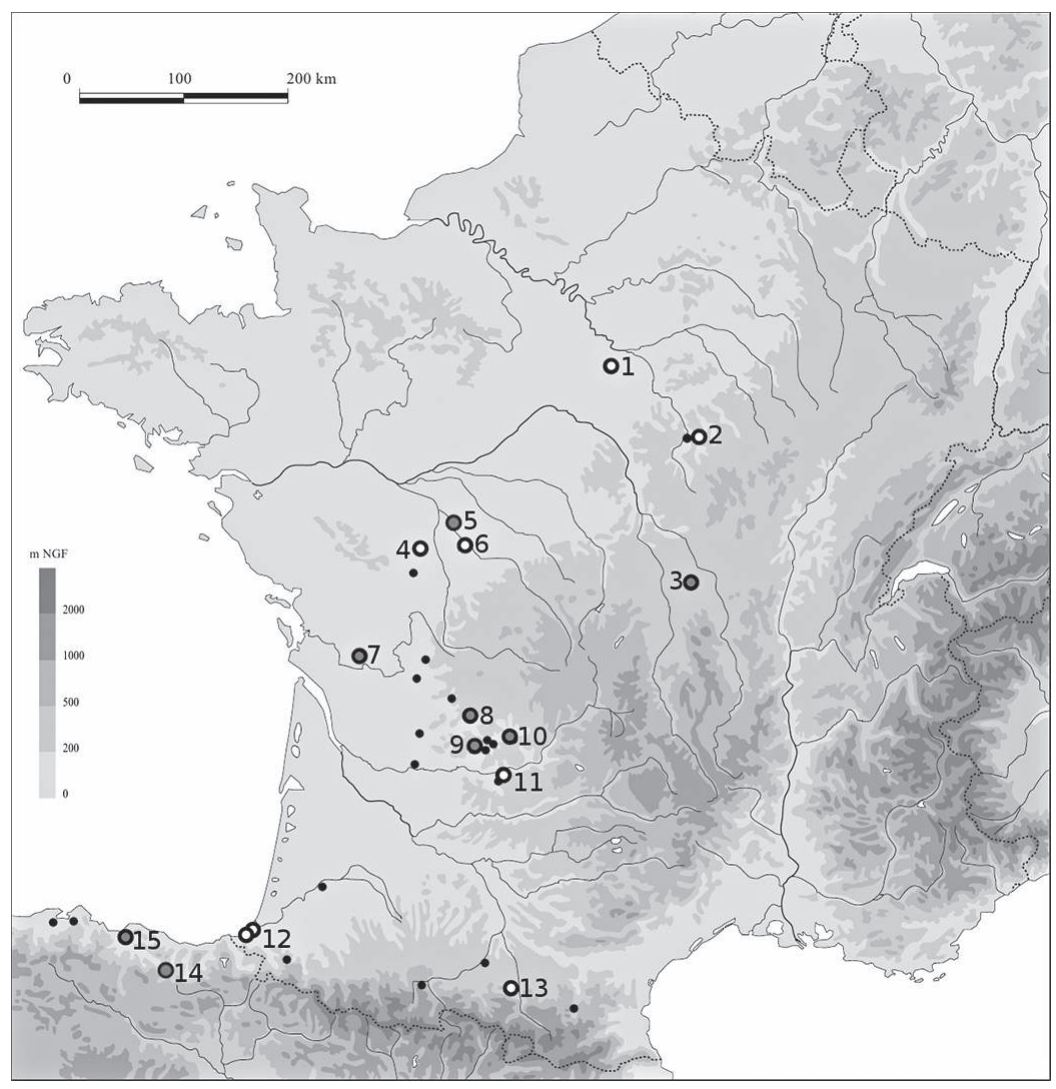

Fig. 39 - Carte de répartition des sites châtelperroniens ou ayant livré des vestiges d'industrie châtelperronienne. Les Oindiquent les sites pour lesquels l'appartenance des matières colorantes au Châtelperronien est avérée. Les O indiquent les sites pour lesquels d'importants mélanges stratigraphiques sont attestés et/ou la documentation archéologique n'est pas suffisante (Tiré de Salomon, 2017 à paraître. Modifié d'après Connet, 2002 ; Dayet et al., 2014). 1) Les Bossats (Ormesson), 2) Grotte du Renne et Grotte du Bison (Arcy-sur-Cure), 3) Grotte des Fées (Châtelperron), 4) La Grande Roche (Quinçay), 5) Abri Fitte-Bordes (Les Roches d'Abilly), 6) Les Cottés (Saint-Pierre-de-Maillé), 7) La Roche à Pierrot (Saint-Césaire), 8) Combe Saunière 1 (Sarliac-sur-I'Isle), 9) Grande Abri de la Ferrassie (Savignac-de-Miremont), 10) Bos-del-Sers (Brivela-Gaillarde), 11) Roc de Combe (Payrignac), 12) Bidart et Le Basté (Saint-Pierre-d'Irube), 13) Les Tambourets (Couladère), 14) Labeko Koba (Arrasate-Mondragón), 15) Aranbaltza (Barrika; DAO : H. Salomon, ULg).

Distribution map of the sites where remains of Châtelperronian industry have been found. The O's indicate the sites where colouring materials are undoubtedly associated with Châtelperronian remains. At the sites marked with $O$, the stratigraphy is disturbed and/or the documentation is not sufficient (after Salomon, 2017 to be published. Modified after Connet, 2002; Dayet et al., 2014). 1) Les Bossats (Ormesson), 2) Grotte du Renne and Grotte du Bison (Arcy-sur-Cure), 3) Grotte des Fées (Châtelperron), 4) La Grande Roche (Quinçay), 5) Abri Fitte-Bordes (Les Roches d'Abilly), 6) Les Cottés (Saint-Pierre-de-Maillé), 7) La Roche à Pierrot (Saint-Césaire), 8) Combe Saunière 1 (Sarliac-sur-I'Isle), 9) Grande Abri de la Ferrassie (Savignacde-Miremont), 10) Bos-del-Sers (Brive-la-Gaillarde), 11) Roc de Combe (Payrignac), 12) Bidart and Le Basté (Saint-Pierre-d'Irube), 13) Les Tambourets (Couladère), 14) Labeko Koba (Arrasate-Mondragón), 15) Aranbaltza (Barrika; CAD: H. Salomon, ULg).

n'avons pas encore identifiés. Autour de cette zone dépotoir et dans un rayon de moins de deux mètres vers le nord et l'ouest (l'est et le sud ne sont pas documentés pour l'instant), se seraient tenues un certain nombre d'activités qui auraient impliqué essentiellement des outils coupants (lames, couteaux de Châtelperron) et des burins (mètres $\mathrm{B}-\mathrm{C} / 33$ et $\mathrm{B}-\mathrm{D} / 34$ ). Plus au nord et à l'ouest (A-D/31, A-D/32, A-B/33 et $\mathrm{A} 34)$, une nette raréfaction des vestiges tranche avec la zone préalablement décrite. S'agit-il des limites de l'occupation châtelperronienne dans ces deux directions? Cela reste à démontrer par de futures fouilles. La suite de l'occupation châtelperronienne se trouve à l'évidence au sud de la surface actuellement fouillée, mais aussi à l'est où près d'une quarantaine de $\mathrm{m}^{2}$ peuvent avoir conservé les traces de cette installation.
Une autre question reste posée concernant la nature de l'extension du niveau châtelperronien vers le nord de la parcelle. Si le sondage 50 a montré que ce niveau existait bel et bien, même s'il reste à démontrer qu'il s'agit bien de la même occupation châtelperronienne que celle du sondage 3 , la faible surface fouillée rend difficile toute interprétation de cet endroit. On notera cependant qu'à côté des nombreux silex taillés dont des outils, parmi lesquels certains sont brûlés, ont été identifiés deux fragments de calcite brûlés, et des esquilles d'os brûlés, ces différents types de témoins indiquant la proximité d'une aire de combustion, ainsi qu'un fragment de matière colorante (supra). Comme dans le sondage 3, on peut donc s'attendre à ce que la zone du sondage 50 soit une aire d'activité et de rejet. Il restera à démontrer alors la contemporanéité et la complémen- 


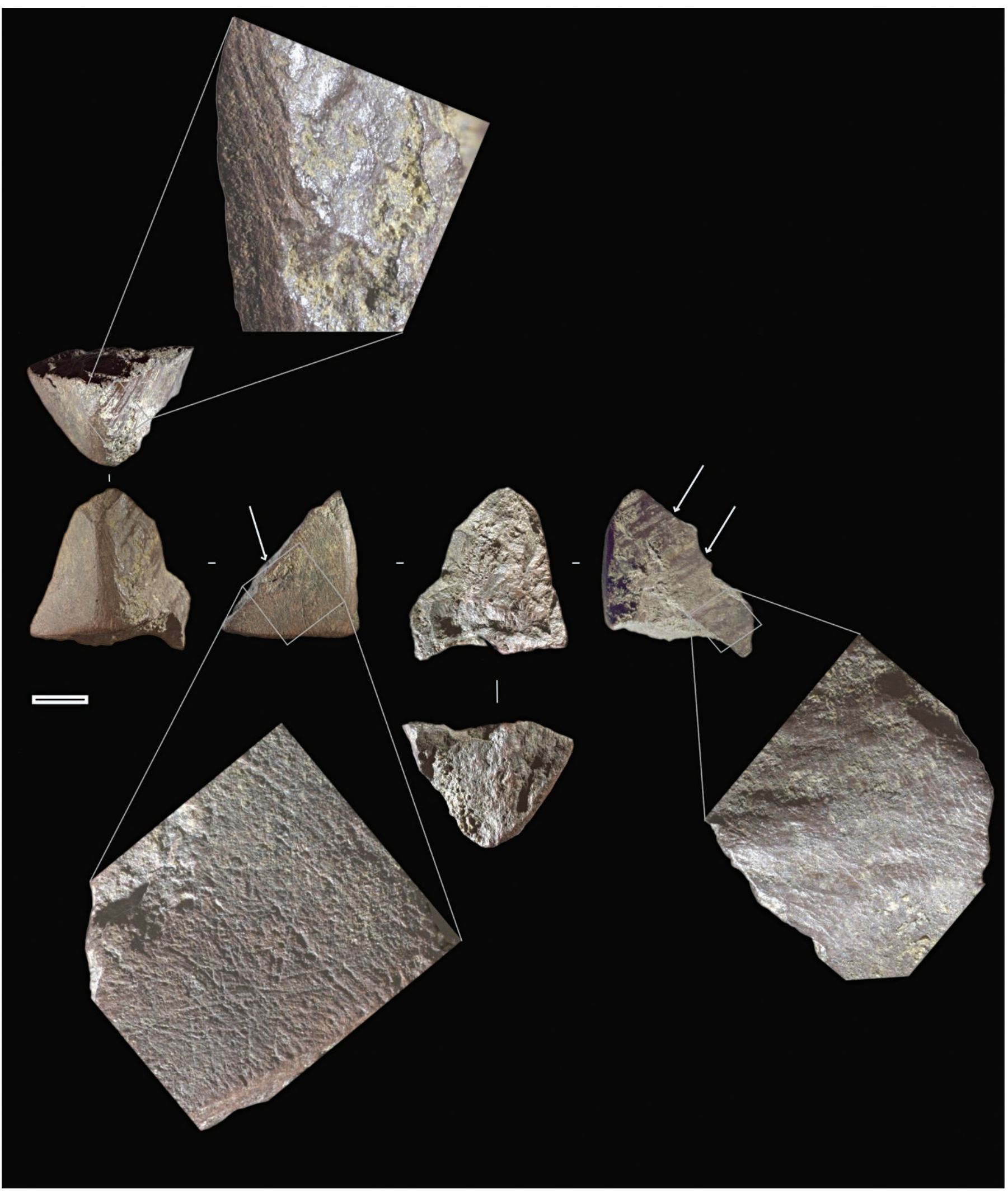

Fig. 40 - Matière colorante facettée du niveau châtelperronien, présentant des stries et des zones lustrées (C): H. Salomon, ULg). Facetted piece of colouring material from the Châtelperronian level, showing striations and a shiny surface (@): H. Salomon, ULg). 
tarité éventuelles entre ces deux espaces distants, rappelons-le, de 15 mètres. D'autres témoins plus discrets plaident en faveur d'une extension du niveau châtelperronien au-delà de ces deux sondages (fig. 5). Le sondage 32 tout au nord-ouest de la parcelle a livré dans un niveau sableux un charbon de bois daté de $37281 \pm 637$ BP (tabl. II). Dans le sondage 34, distant d'environ 7 mètres au nord-ouest du sondage 50 , deux objets taillés d'aspect laminaire ont été découverts dans un niveau sableux évoquant celui dans lequel repose le Châtelperronien. Enfin un dernier sondage, le 31 , situé à 5 mètres au sud-est du sondage 50, a également donné dans un même niveau sableux quatre objets lithiques dont un éclat laminaire semi-cortical. Ce sont là des témoignages bien modestes, mais ils permettent, pour le moment et en l'attente de fouilles de grande ampleur, de proposer une extension du niveau châtelperronien sur environ $300 \mathrm{~m}^{2}$, ce qui est une surface tout à fait respectable pour un gisement châtelperronien de plein air, de surcroît situé au nord de la Loire.

\section{DES COMPARAISONS PLUS OU MOINS LOCALES?}

La quasi-absence de gisements châtelperroniens au nord de la Loire est en effet un constat relativement ancien que la découverte du gisement d'Ormesson permet de questionner (fig. 18). Cet état de fait semble en effet plus lié à un état de la recherche qu'à une réelle absence. La simple observation de la carte des gisements châtelperroniens présentée par M. Soressi et $\mathrm{M}$. Roussel dans une synthèse récente (Roussel et Soressi, 2014) indique une rupture trop nette, selon nous, entre une large diagonale sud-ouest/centre-est où les gisements châtelperroniens sont assez nombreux et toute la partie située au nord de la Loire où les occurrences châtelperroniennes connues se réduisent aux cavités d'Arcy-sur-Cure et de la Roche-au-Loup (Merry-sur-Yonne) situées à peu de choses près à la même latitude que la Loire. Mais cette frontière nous semble trop tranchée pour refléter la réalité. On devait s'étonner d'ailleurs que les grottes d'Arcy-sur-Cure et celle très proche de la Roche-au-Loup (Parat, 1904; Breuil, 1911 ; Poplin, 1981, 1982, 1983a, 1983 b, 1984, 1985, 1986a, 1987) soient les seuls indices de la présence châtelperronienne la plus septentrionale. Et cela alors même que le réseau hydrographique qui les borde se prolonge au nord dans la vallée de l'Yonne qui elle-même rejoint la Seine à Montereau en Seine-et-Marne. Il y a là un vaste potentiel de déplacements vers des régions où la matière première siliceuse, entre autres, est abondante et où les territoires de chasse n'ont pas manqué comme le prouve l'intensité de certaines occupations du Paléolithique supérieur entre autres (Gravettien, Badegoulien, Magdalénien final). L'origine même des silex apportés à Arcy et sans doute à la Roche-auLoup pour certaines catégories est à rechercher plus au nord, à minima, dans le Jovinien dans l'Yonne où les sites du Paléolithique supérieur sont également nombreux, à l'exception de ceux attribués au Châtelperronien. Ainsi dans le cadre d'un projet collectif de recherche menée entre 1999 et 2005 (Bodu et al., 2013c), il a été possible de montrer, à la suite des travaux de B. Schmider (1971), que des occurrences aurignaciennes, gravettiennes, solutréennes, mais aussi du Magdalénien ancien et moyen étaient nombreuses entre l'Auxerrois et le Sénonais, le Châtelperronien faisant office de grand absent dans ce cortège. À l'exception peut-être d'un indice, certes moyennement fiable, provenant d'Auxerre (Yonne) ou de sa périphérie immédiate. Il s'agit d'une petite série lithique d'une trentaine de pièces déposée dans les réserves du musée d'Avallon et identifiée comme provenant d'une sablière dite «Portail» à Auxerre. $\mathrm{Au}$ sein de la série d'aspect plutôt laminaire se trouvent trois pièces à dos qui pourraient s'apparenter à des couteaux de Châtelperron, mais il n'est pas exclu que l'on ait affaire à des objets plus récents (Gravettien, Azilien? ; fig. 41). En raison de l'incertitude qui pèse sur cette série, dont les conditions de récolte nous sont inconnues, ce sont les déplacements effectués par les châtelperroniens d'Arcy-sur-Cure et de Merry-surYonne pour le silex qui sont donc les meilleurs indices, même si indirects, de leur fréquentation de territoires situés plus au nord de la Bourgogne. Dès lors l'absence de sites châtelperroniens au nord d'Arcy-sur-Cure doit être considérée comme une lacune dans la documentation. Ce biais peut être imputé certes à la relative faible fréquence du Châtelperronien entre Loire et Manche, mais il est à peu près certain que cette industrie, peu caractéristique au premier abord si on lui ôte ses fameux couteaux ou pointes, est passée inaperçue ou n'a pas été identifiée dans des séries recueillies en surface notamment, voire a été confondue avec d'autres ensembles lithiques non contemporains. Et à l'exception d'Arcy-sur-Cure, il n'existe quasiment

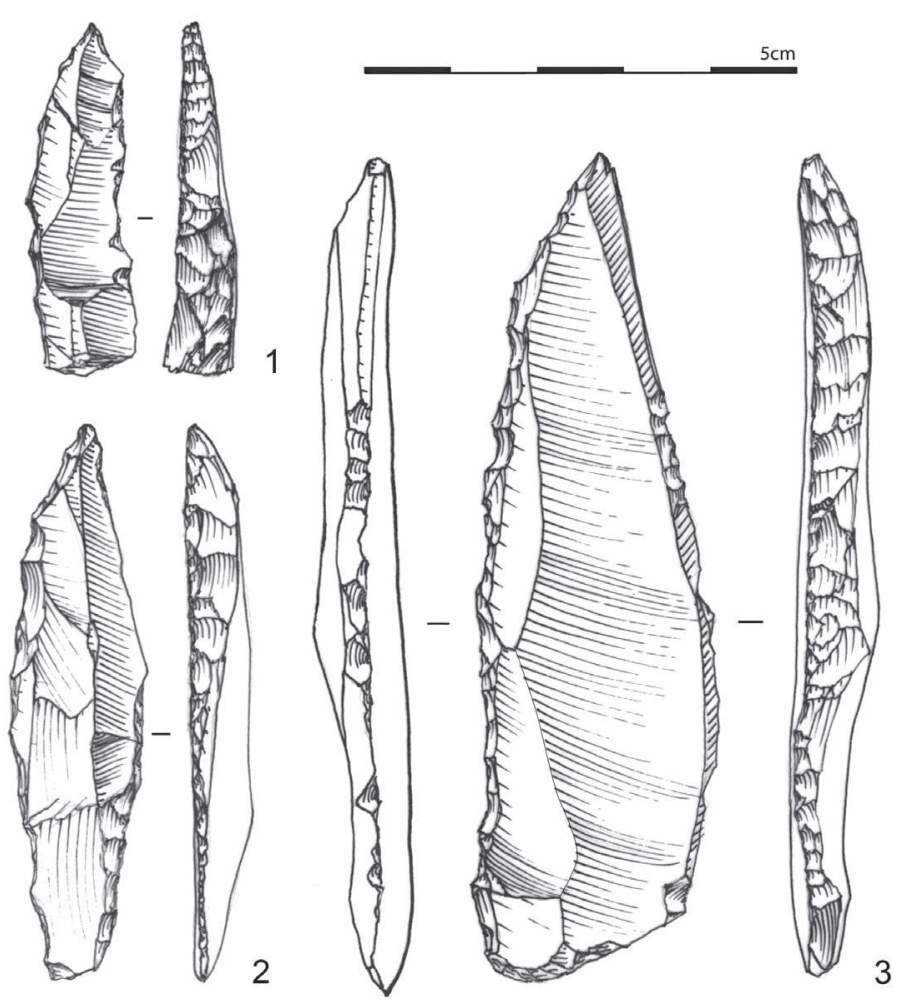

Fig. 41 - Trois pièces à dos provenant de la carrière Portail à Auxerre (Yonne; DAO : M. Ballinger, CNRS).

Three backed pieces from the Portail quarry at Auxerre (Yonne; CAD: M. Ballinger, CNRS). 
pas, au nord de la Loire, de gisements à stratigraphie complexe où différentes occupations du Paléolithique supérieur seraient superposées et où le Châtelperronien, s'il existait, pourrait être plus facilement reconnu. D'autre part, malgré un intérêt relativement précoce pour la préhistoire ancienne dans le Bassin parisien et plus généralement toute la partie nord de la France, de la part d'amateurs plus ou moins éclairés ou de rares chercheurs, les gisements rapportés au Paléolithique supérieur ancien restent assez peu nombreux dans cette vaste région. Il n'est dès lors pas surprenant que le Châtelperronien ne soit pas très connu au nord de la Loire $^{20}$. L'exemple de la Roche-au-Loup à Merry-sur-Yonne (Bourgogne) est symptomatique d'une certaine méconnaissance de la présence châtelperronienne au nord de la Loire. Le site est ainsi évoqué dans quelques rares travaux de synthèse (Roussel et Soressi, 2014) et articles ciblés sur certains aspects (Poplin, 1983 b, 1986 b, 1986c, 1988), mais peu de descriptions détaillées sont faites du matériel archéologique, notamment lithique, qui y a été trouvé. On en sait peu malgré la qualité et la quantité de la documentation recueillie par l'Abbé Parat et surtout par F. Poplin (Poplin, 1981, 1982, 1983a et b, 1984, 1985 1986a,b,c 1987, 1988). L'Abbé Parat fouille ce gisement dans les années 1900, mais n'identifie que deux niveaux d'occupation, l'un qu'il attribue au Moustérien, l'autre au Magdalénien. Il ne fait pas état de la découverte de Châtelperronien et écrit même ne pas observer «comme à la grotte de l'Ours, à Arcy, le passage d'une industrie à l'autre» ${ }^{21}$ (Parat, 1904). Les descriptions qu'il fait de la série recueillie dans le niveau moustérien épais «de 1 à 2 mètres» évoquent cependant la possibilité d'une présence châtelperronienne («Plusieurs échantillons ont la régularité d'un triangle isocèle, mais généralement un des bords latéraux fait une courbe. Plusieurs spécimens se terminent en pointe acérée qui paraît ménagée pour percer tandis que les bords retouchés servaient à racler» [Parat, 1904]), ce que confirmera plus tard l'Abbé Breuil en reprenant la série (Breuil, 1911). Dans un article consacré à l'industrie de la grotte de Châtelperron et d'autres gisements similaires, Breuil présente le matériel découvert par Parat à la Roche-au-Loup. Il y figure notamment de nombreuses «pointes à retouches unilatérales du type de Châtelperron» typiques (fig. 42), mais la possibilité d'un mélange avec d'autres industries du Paléolithique supérieur n'est pas à écarter ${ }^{22}$. Ce sont "plusieurs beaux couteaux de

20. Une simple anecdote permet de se rendre compte de la difficulté à identifier le matériel lithique châtelperronien : découvert en 2010, fouillé en 2011 et 2012, il aura fallu attendre l'année 2013 et la collecte d'un nombre assez élevé de silex taillés, dont les fameuses pointes, pour confirmer l'attribution au Châtelperronien de ce niveau à Ormesson.

21. Entendre de l'industrie à éclats à l'industrie à lames.

22. Bien plus tard, Nelly Connet dans le cadre de ses travaux de thèse et à l'occasion d'une étude menée sur la série Parat déposée au musée
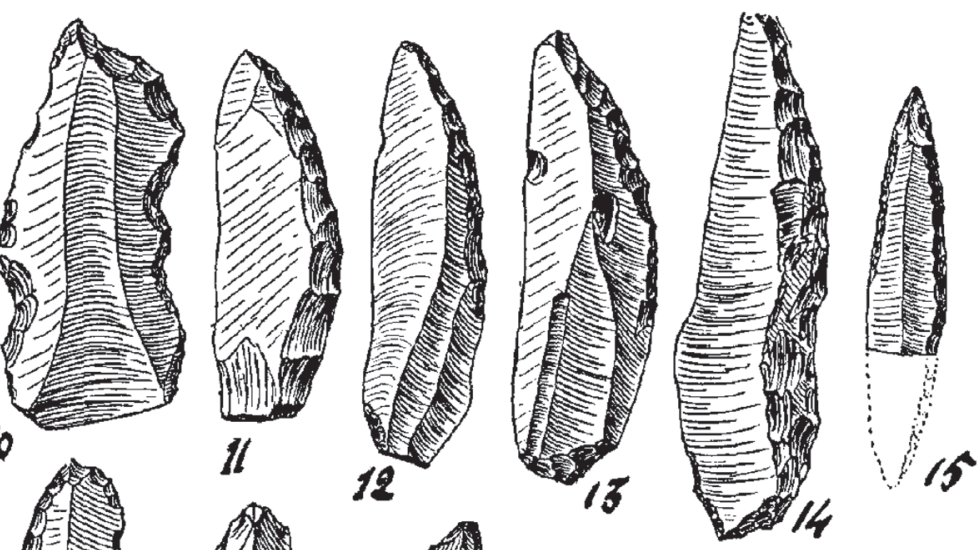

Fig. 42 - Couteaux à dos châtelperroniens du site de la Roche-au-Loup

(d'après Breuil, 1911).
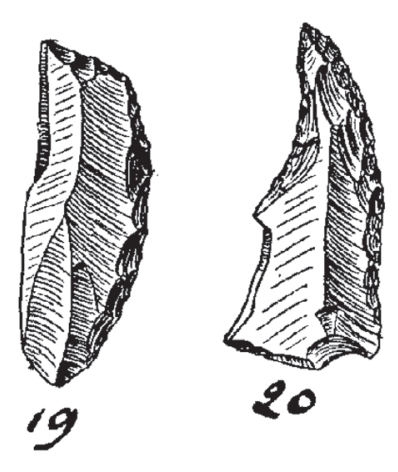

16
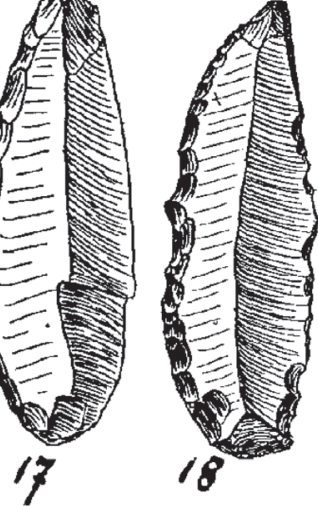

type de Chatelperron, trouvées a la Roche an
Echelle $2 / 3$. Le $\mathrm{n}^{\circ} \mathrm{2} 0$ passe au percoir courbe.

hâtelperronian backed knifes from the site la Roche-au-Loup (after Breuil, 1911).

Châtelperron qui témoignent d'une industrie peu commune du début du Paléolithique supérieur (couches IX et X de la Grotte $\mathrm{du}$ Renne à Arcy-sur-Cure)» que F. Poplin trouve également au début des années 80 lorsqu'il reprend la fouille du gisement de la Roche-au-Loup ${ }^{23}$. L'opération visant à trier et tamiser les déblais de l'abbé Parat lui permet d'identifier et de documenter un lambeau de couche bréchifiée à l'entrée de la cavité qu'il rapproche du Châtelperronien (Poplin, 1983, p. 24; Poplin, 1984, p. 11). Cette attribution sera ensuite confirmée par une campagne de fouille pendant laquelle la même brèche livre quelques éléments supplémentaires identifiables comme étant des couteaux de Châtelperron (Poplin, 1985, p. 28). Nous avons

d'Avallon a également proposé une présence châtelperronienne. Elle avait observé que le silex était majoritaire dans cette série qui comportait certes des éléments du Paléolithique moyen (racloirs en abondance souvent amincis), mais aussi des pointes de Châtelperron, des burins, un nucléus à lamelles et une lamelle retouchée (N. Connet, com. orale).

23. F. Poplin consacrera sept campagnes de fouille à la Roche-auLoup entre 1981 et 1987. Le matériel archéologique est actuellement stocké dans une des réserves du MNHN où nous avons pu prélever temporairement les éléments intéressants dans le cadre de cette enquête. 
pu confirmer et documenter plus amplement cette présence châtelperronienne à la Roche-au-Loup en consultant le mobilier Parat déposé à Avallon et celui provenant des fouilles plus récentes ${ }^{24}$. Étant donné la quantité considérable de matériel lithique recueilli, nous nous sommes contentés de passer rapidement en revue la collection déposée au MNHN afin d'y repérer les pièces les plus diagnostiques ${ }^{25}$. Ce tri rapide de l'industrie lithique nous a permis d'identifier outre quelques couteaux de Châtelperron entiers ou fragmentés ${ }^{26}$ (fig. 43, fig. 44) un assez grand nombre de nucléus en chaille et silex d'exploitation «châtelperronienne» avec un investissement progressif de la tranche vers la surface la plus large du nucléus pour l'obtention de supports laminaires. Ce matériel provient soit des déblais des fouilleurs précédents soit de l'entrée de la grotte où se trouve la brèche évoquée précédemment, soit du centre ou du fond de la cavité où quelques pièces lithiques découvertes par F. Poplin évoquent aussi le Châtelperronien. L'étude de cette série montre, dans un premier temps, que l'occupation châtelperronienne identifiée par Breuil puis F. Poplin était sans doute très riche mais il nous est impossible d'en donner une estimation quantifiée en raison de la médiocre qualité des fouilles réalisées par les prédécesseurs de F. Poplin et des difficultés de ce dernier à retrouver, après leurs opérations, des niveaux en place et facilement attribuables. Dans la mesure où le gisement de la Roche-au-Loup est l'un des très rares sites châtelperroniens situés au nord de la Loire et que peu d'articles ont été consacrés au détail de l'industrie lithique châtelperronienne, nous avons jugé nécessaire d'illustrer par la reprise de dessins plus ou moins anciens et par de nouvelles représentations graphiques certains objets lithiques caractéristiques. Malgré la médiocre qualité des contextes de découverte, l'occupation châtelperronienne de la Roche-au-Loup représente donc un témoin majeur pour la connaissance de cette période au nord de la Loire ${ }^{27}$. Ainsi retrouver l'origine des matières

24. Dans ces rapports très détaillés ainsi que quelques articles, F. Poplin fait état de la découverte d'objets travaillés en os, en bois de cervidé ou en ivoire, d'éléments de parure et de curios, dont certains pourraient être rapportés au niveau châtelperronien. Des restes humains fragmentaires sont même découverts, mais le contexte de la découverte est incertain même si l'un d'entre eux (une dent) semble provenir de la brèche attribuée, pour partie, au Châtelperronien (Poplin, 1986, p. 38 et 106).

25. Les occupations préhistoriques de la Roche-au-Loup concernent à minima le Paléolithique moyen, le Châtelperronien, le Gravettien et vraisemblablement le Magdalénien ancien/Badegoulien ainsi que le Magdalénien moyen ou final (Poplin, 1981 à 1987 et observations personnelles).

26. Lors de notre premier tri des éléments châtelperroniens diagnostiques dans la réserve du MNHN, il ne nous a pas été possible de retrouver quelques couteaux de Châtelperron évoqués et parfois représentés par F. Poplin dans certains de ses rapports soit qu'ils nous aient échappé, soit qu'ils se trouvent à un autre endroit. Nous avons en revanche identifié quelques autres couteaux et assimilés non figurés dans les rapports.

27. Témoin qui mériterait une étude plus détaillée du corpus lithique, mais aussi des datations sur un certain nombre de restes osseux découverts «en relation» avec des pièces typiquement châtelperroniennes.
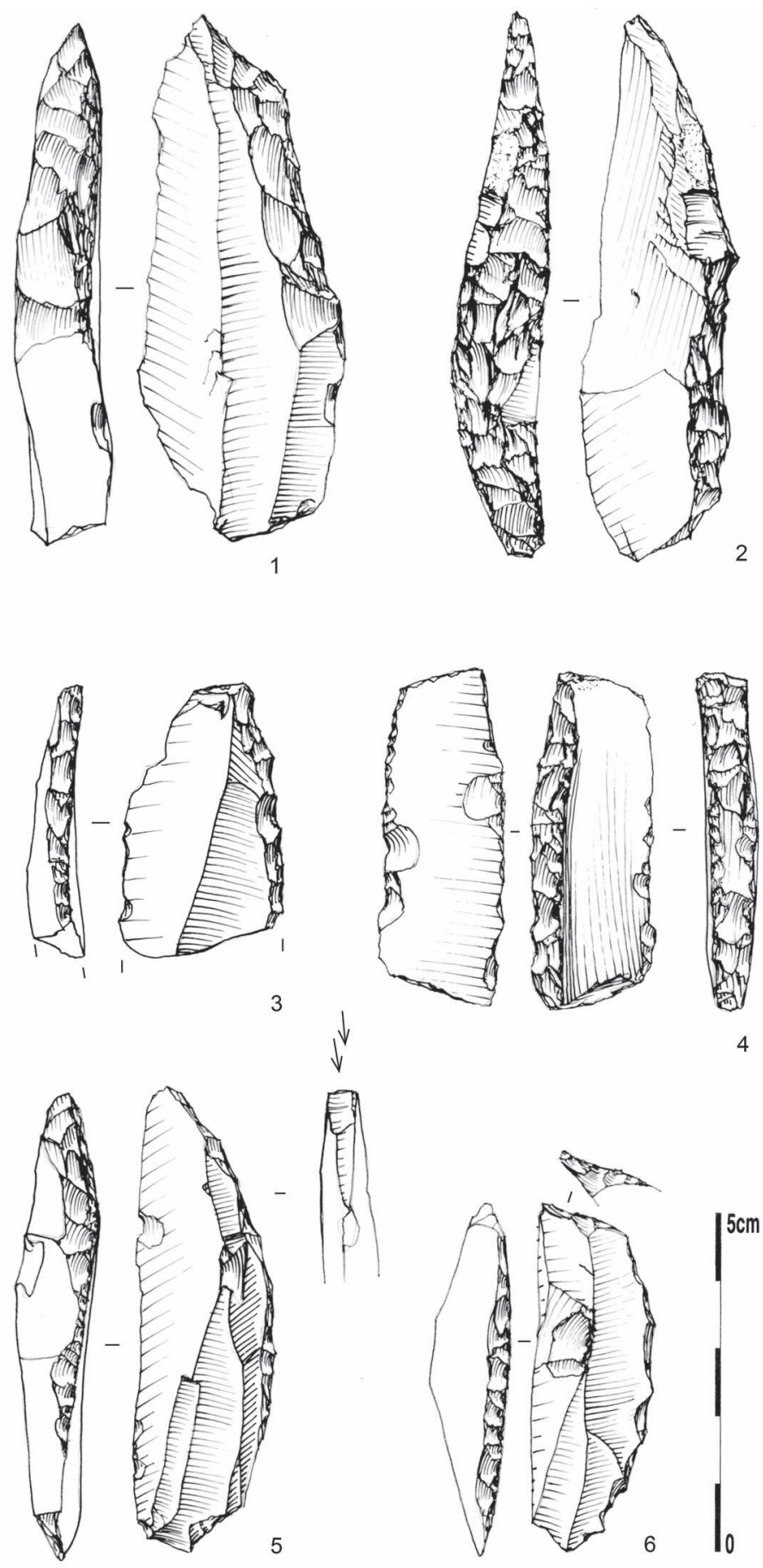

Fig. 43 - Couteaux à dos châtelperroniens du site de la Roche-auLoup, fouilles F.Poplin (DAO : M. Ballinger, CNRS).

Châtelperronian backed knives from the site La Roche-au-Loup, excavations by F. Poplin (CAD: M. Ballinger, CNRS). 

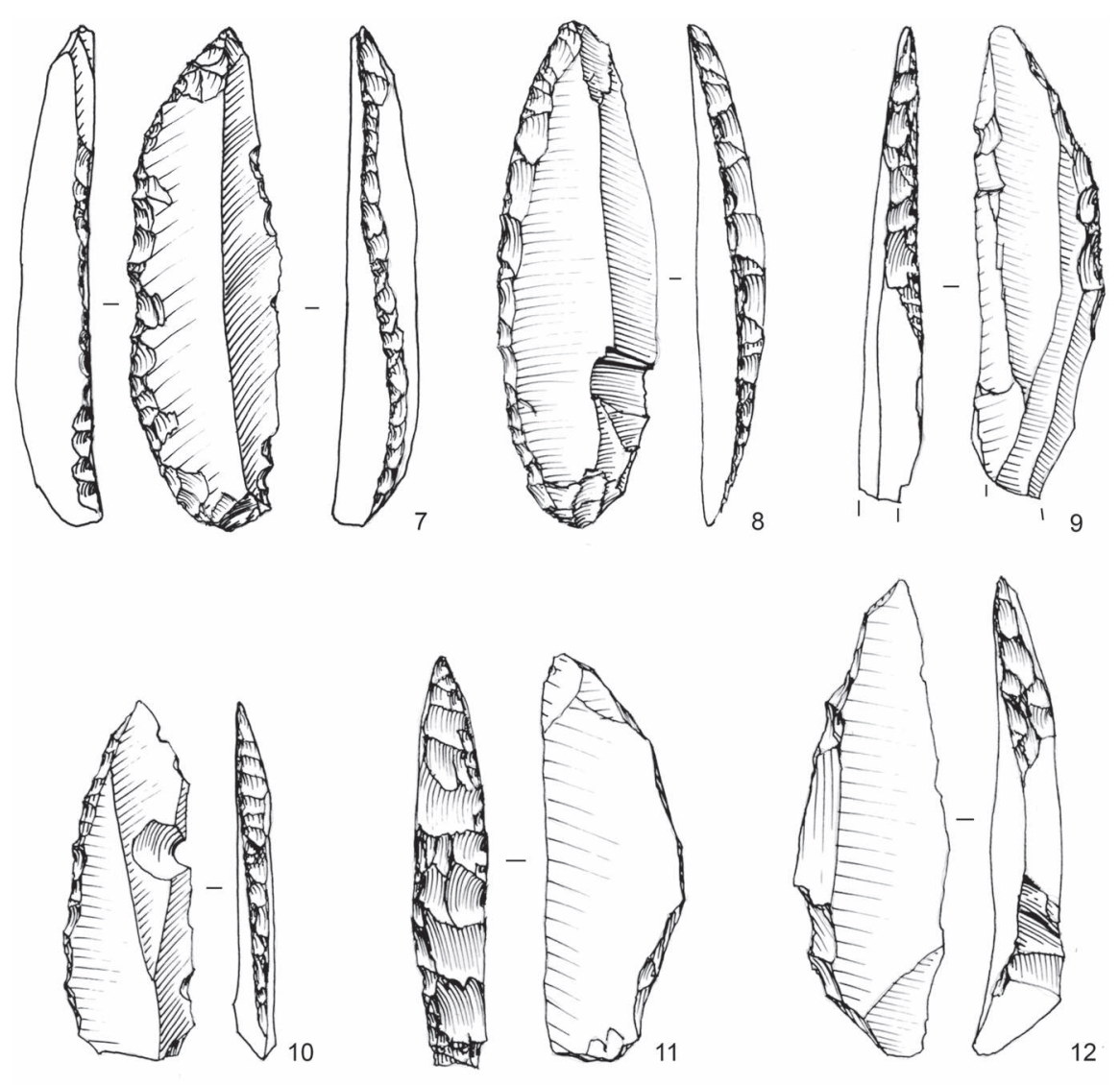

Fig. 44 - Couteaux à dos châtelperroniens du site de la Roche-au-Loup, fouilles F. Poplin (DAO : M. Ballinger, CNRS).

Châtelperronian backed knives from the site La Roche-au-Loup, surveys by F. Poplin (CAD: M. Ballinger, CNRS).

pointes à dos courbe magdaléniennes ou aziliennes selon leur état de patine et leurs dimensions qui complètent un ensemble de pièces véritablement tardiglaciaires que R. Daniel avait découvert dans ce même gisement. Une autre pièce provenant du bois des Beauregards à Nemours et présentée par Ch. Vaché comme étant une "pointe type Châtelperron» (Vaché, 1932) ${ }^{28}$ (fig. 46) semble aussi peu convaincante d'autant plus que sa position stratigraphique s'apparente à celles des objets badegouliens découverts par milliers dans ce même gisement, à moins que cet objet, comme les précédents, ne soit une pièce à dos azilienne. Ainsi, dans la vallée du Loing située à moins de $5 \mathrm{~km}$ à l'est d'Ormesson, aucun gisement châtelperronien n'a été identifié à l'heure actuelle et ce malgré l'importante quantité de sites d'autres périodes appartenant au Paléolithique moyen, mais aussi au Paléolithique supérieur (essentiellement Gravettien et Badegoulien), découverts depuis le $\mathrm{XIX}^{\mathrm{e}}$ siècle notamment au Bois des Beauregards ou encore au Cirque de la Patrie (Cheynier, 1962; Schmider, 1971; Schmider et Roblin-Jouve, 2008). Certes, l'absence de gisements aurignaciens dans la vallée du Loing, par ailleurs assez nombreux dans les Yvelines et dans l'Yonne proches (Bodu et al., 2013 b), doit nous alerter, car elle a peut-être pour cause des processus taphonomiques qui auraient pu contribuer à fortement entamer ou simplement éliminer les occupations antérieures au Gravettien. Ce qui a pu arriver aux sites aurignaciens de la vallée du Loing aurait pu tout aussi bien concerner des occupations châtelperroniennes.

premières lithiques transportées par les Châtelperroniens de la Roche-au-Loup serait un très bon moyen pour reconstituer leurs déplacements notamment vers le nord. Plus près d'Ormesson, les indices du Châtelperronien sont donc absents et ce ne sont pas les quelques pièces découvertes dans la grotte du Troglodyte à Nemours par M. et R. Daniel qui permettent de pallier cette absence. Ces deux auteurs ont ainsi publié quatre pièces qu'ils identifient comme appartenant au Périgordien I, soit au Châtelperronien (Daniel et Daniel., 1953 ; fig. 45). Nous avons pu retrouver ces quatre pièces au Musée des Antiquités nationales à Saint-Germain-en-Laye. Il s'agit en réalité de
Force est donc de constater qu'à l'exception des gisements d'Arcy-sur-Cure et de la Roche-au-Loup à Merry-sur-Yonne, aucun des rares autres gisements sous abri situés au nord de la Loire, n'a livré de vestiges d'une quelconque occupation châtelperronienne y compris les grottes de la vallée de l'Erve en Mayenne, situées à la même latitude qu'Ormesson et plus connues pour leurs occupations aurignaciennes, gravettiennes (Rochefort, Mayenne-Science) et surtout du Solutréen moyen (Allard, 1985; Hinguant et Biard, 2013).

28. La localisation actuelle de cet objet nous est inconnue. 
Fig. 45 - Quatre pointes aziliennes du site de la grotte du Troglodyte, Nemours (d'après R. Daniel, 1953 ; $D A O:$ M. Ballinger, CNRS).

Four Azilian points from La grotte du Troglodyte, Nemours (after R. Daniel, 1953; CAD: M. Ballinger, CNRS).

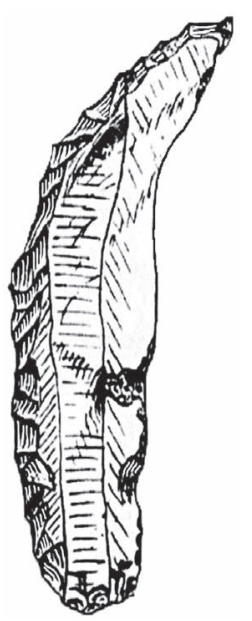

Fig. 46 - Une pièce à dos identifiée comme couteau de Châtelperron provenant du site des Beauregards à Nemours (environ $9 \mathrm{~cm}$ de longueur, d'après Vaché, 1932).

A backed piece identified as a Châtelperronian knife from the site Les Beauregards at Nemours (approximately $9 \mathrm{~cm}$ in length, after Vaché, 1932).

Plus au sud-est, l'occupation initialement considérée comme châtelperronienne(Desbrosse, 1984) du trou de la Mère-Clochette à Rochefort-sur-Nenon (Jura), situé à $7 \mathrm{~km}$ au nord-ouest de Dôle, a finalement été réattribuée au Gravettien (Bachellerie et Brou, 2009; Szmidt et al., 2010). En revanche, E. Dutkiewicz et H. Floss ont pu récemment réhabiliter, par des recherches documentaires et des fouilles, les occupations châtelperroniennes des grottes de la Verpillière I et II à Germolles (Mellecey, Saône-et-Loire ; Floss et al., 2013 ; Dutkiewicz et Floss, 2015) ${ }^{29}$. Mais nous restons là encore dans un espace géographique assez classique pour le Châtelperronien (fig. 18).

\section{CONCLUSION}

Ainsi le gisement d'Ormesson apparaît comme étant particulièrement exceptionnel dans ce «désert» qui caractérise l'occupation châtelperronienne au nord de la Loire. Cela est d'autant plus vrai qu'il s'agit d'un gisement de plein air où le risque de mélanges avec des niveaux plus anciens ou plus récents est tout à fait minime contrairement à ce qui se passe dans bien des

29. Au moment où nous bouclons ce manuscrit, un article concernant l'occupation châtelperronienne des grottes de la Verpillière est en cours de validation pour publication (Paléo). Nous renvoyons bien évidemment les lecteurs à sa consultation. abris. Les résultats présentés ici sont certes préliminaires, mais ils permettent déjà de poser plusieurs jalons quant à la compréhension d'un certain nombre de phénomènes liés aux modalités de production (et d'utilisation?) des équipements en silex et à l'usage de matières colorantes par les Châtelperroniens. La part du lamellaire au sein de cette industrie, comme cela avait été observé dans d'autres séries du Sud-Ouest (Roussel, 2011 ; Roussel et al., 2016; Bachellerie, 2011), semble particulièrement importante même si nous n'avons pas encore précisé la fonction de ces produits. Malgré leur nombre particulièrement réduit, il semble néanmoins que des microlithes retouchés aient constitué un des objectifs du débitage des lamelles même si nous n'excluons pas l'utilisation de tranchants bruts. Nous envisageons également que les lamelles retouchées d'Ormesson puissent être une déclinaison plus ou moins réduite des couteaux à dos. Cela reste à confirmer par une analyse tracéologique ultérieure et la découverte d'un plus grand nombre d'exemplaires de lamelles retouchées, ou non.

Quoi qu'il en soit l'entrée des Châtelperroniens dans le monde lamellaire est un phénomène qui doit être mieux compris encore puisque porteur d'éléments de réflexion d'ordre anthropologique. Les auteurs du Châtelperronien ont-ils été influencés par d'autres groupes humains dans leur pratique du débitage des roches siliceuses, notamment pour la production de lamelles? Le silex est un matériau certes prolixe, mais il est des domaines que son étude, même poussée, ne permet pas ou permet difficilement, d'aborder. Cependant, on peut proposer que la proximité technique entre le schéma lamellaire et le schéma laminaire, observée à Ormesson, traduise bien l'appartenance des deux productions à une communauté de conceptions qui ne s'est pas nécessairement inspirée d'exemples extérieurs. Après tout, les Moustériens du Nord de la France comme ceux du gisement de Riencourt-lès-Bapaumes (série CA, stade isotopique 5a; Tuffreau dir., 1993; Vande Walle, 2003) ont bien produit des lamelles et retouché certaines d'entre elles, même si le terme lamelle même n'est pas utilisé par les auteurs (voir par ex. Tuffreau, dir. 1993, p. 27, 29, 40 et 44). Dans les sites bourguignons de la région de Sens (Lailly «Beauregard», Molinons), certaines industries du Paléolithique 
moyen récent ont, elles aussi, livré quelques indices de production lamellaire (Depaepe, 2007). On voit bien, ce faisant, que l'interrogation, légitime, sur l'origine de la production lamellaire au Châtelperronien requiert une meilleure connaissance des schémas de production des lamelles au Moustérien, tout comme il convient d'affiner les comparaisons avec le Proto-Aurignacien. On ne peut pas suspecter, à Ormesson, de mélange entre les silex châtelperroniens et du matériel lithique de périodes plus récentes. La sophistication du débitage laminaire, déjà bien décrite dans d'autres gisements, est donc indiscutable et c'est un caractère fort qui signe une rupture très nette avec ce qui se passe auparavant. La genèse de la mise en place d'un débitage laminaire si abouti au Châtelperronien, peu de temps après les dernières manifestations moustériennes, doit être, comme celle des lamelles, mieux documentée. On peut compter désormais sur une analyse particulièrement fine de la production laminaire châtelperronienne d'Ormesson grâce aux remontages que l'on sait d'ores et déjà très potentiels, afin de la comparer avec les productions laminaires moustériennes (Révillon et Tuffreau, 1994; Blaser et al., 2012). Pour le moment, le niveau châtelperronien d'Ormesson n'a été fouillé que sur un peu moins d'une vingtaine de $\mathrm{m}^{2}$ alors qu'on estime son extension minimale à près de $150 \mathrm{~m}^{2}$. Il est dans nos projets, une fois les occupations solutréennes et gravettiennes traitées, de poursuivre l'exploration de ce niveau unique par bien des aspects. C'est en effet à l'aune d'un vaste décapage, à l'instar de ce qui est réalisé pour le Magdalénien ou l'Azilien du Bassin parisien par exemple, que nous pourrons développer une véritable approche palethnologique des Châtelperroniens. À une échelle plus vaste que celle du site, la découverte d'Ormesson pose la question de l'extension septentrionale de cette entité chronologique et nous amène à réinterroger certains ensembles lithiques du Bassin parisien à composante laminaire ubiquiste non ou mal attribués. Puisque le niveau châtelperronien d'Ormesson existe, il est évident que d'autres occurrences châtelperroniennes nous attendent plus au nord encore!

\section{Remerciements}

Les auteurs tiennent à remercier les deux rapporteurs qui ont contribué notablement à l'amélioration du manuscrit. Ils remercient aussi très chaleureusement Rebecca Miller et Yan-Axel Gomez-Coutouly pour la traduction du résumé en anglais et Noora Taipale ainsi qu'Emmanuelle Honoré pour la correction de la version anglaise des légendes, Colas Guéret pour les clichés des fragments d'os striés ainsi que Marie Soressi et Morgan Roussel pour leur autorisation d'utilisation de la carte de répartition des gisements châtelperroniens. Nos remerciements s'adressent également à Margareta Tengberg et au Service régional de l'Archéologie de BourgogneFranche-Comté qui nous ont facilité l'accès aux collections de la Roche-au-Loup (fouilles François Poplin) et au Musée d'Avallon pour l'accès aux séries de l'Abbé Parat. Nous remercions le propriétaire de la parcelle du site les Bossats, Didier Lebègue qui nous accorde très aimablement son autorisation depuis 2009, le Service régional de l'Archéologie d'Ile-de-France pour son accompagnement financier et scientifique, le Conseil départemental de Seine-et-Marne pour son aide financière, le Parc Naturel régional du Gâtinais, la mairie d'Ormesson et son maire Alain Poursin pour leur soutien indéfectible et la communauté de communes du pays Nemourois, Christian Peutot en particulier, qui collabore efficacement au projet Ormesson. Enfin, un très grand merci s'adresse à l'ensemble des fouilleurs et spécialistes qui participent à l'exploitation scientifique de ce gisement. 


\section{BIBLIOGRAPHIE}

\section{Allard M.}

1985 : «Solutréen de Thorigné-en-Charnie et de Saint-Pierre-sur-Erve (Mayenne)», Bulletin de la Société préhistorique française, 82 (fasc. 10-12), p. 338-349.

Armand D., Plassard F., Prat F.

2004: L'Ours de Font-de-Gaume III et le problème de l'exploitation de l'Ours des Cavernes, Cahiers scientifiquesMuséum d'histoire naturelle de Lyon, p. 103-110.

Aubry T., Dimuccio L., Almeida M., Buylaert J.-P., Fontana L., Candela P., Higham T., Liard M., Murray A.S., Neves M.J., Peyrouse J.-B., Walter B.

2012: 'Stratigraphic and technological evidence from the middle palaeolithic-Châtelperronian-Aurignacian record at the Bordes-Fitte rockshelter (Roches d'Abilly site, Central France)', Journal of Human Evolution, 62 (issue 1), p. 116-137.

Aubry T., Almeida M., Candela P., Chauviere F.-X., Dimuccio L., Fontana L., Liard M., Marquet J.-C., Neves M.J., Peyrouse J.-B., Walter B.

2013: «Le Paléolithique supérieur ancien dans le sud-ouest du Bassin parisien : du Châtelperronien au Gravettien dans les vallées de la Creuse et de la Claise» in Bodu P., Chehmana L., Klaric L., Mevel L., Soriano S., Teyssandier N. (dir.), Le Paléolithique supérieur ancien de l'Europe du NordOuest (35000-15000 BP) : réflexions et synthèses à partir d'un projet collectif de recherche sur le Paléolithique supérieur ancien du Bassin parisien, actes du colloque, Sens, avril 2009, Société préhistorique française, Mémoire LVI, Paris, p. 299-315.

Bachellerie F., Bordes J.-G., Morala A., Pelegrin J.

2007: «Étude typo-technologique et spatiale de remontages lithiques de Canaule II, site châtelperronien de plein air en Bergeracois (Creysse, Dordogne)», Paléo, 19, p. 259-280.

Bachellerie F., Brou L.

2009: "Les Châtelperroniens ont-ils été jurassiens? Diagnostic chronoculturel de "l'industrie à pièce à dos" du Trou de la Mère Clochette (Rochefort-surNenon)», in Jacottey L., Milleville A. (dir.), Gestion des Matières Premières et Implantation Humaine Autour du
Massif de la Serre (39). Projet Collectif de Recherche 2007-2009, Rapport 2009, inédit, Service Régional d'archéologie de Franche-Comté, p. 241-246.

\section{BaChellerie $\mathbf{F}$.}

2011 : Quelle unité pour le Châtelperronien? Apport de l'analyse taphonomique et techno-économique des industries lithiques de trois gisements aquitains de plein air : le Basté, Bidart (PyrénéesAtlantiques) et Canaule II (Dordogne). Thèse de doctorat, Université de Bordeaux I, Talence, inédit, $442 \mathrm{p}$.

Bachellerie F., Bon F., Deschamps M., Eizenberg L., Henry-Gambier D., Mourre V., Normand C., Pelegrin J., Primault J., Scandiuzzi R., Thiebaut C.

2011: «La signature archéologique de l'activité de chasse appliquée à la comparaison des industries moustériennes, châtelperroniennes et aurignaciennes des Pyrénées : nature des équipements et fonctions des sites», in BoN F., Costamagno S., Valdeyron N. (dir.), Haltes de chasse en Préhistoire. Quelles réalités archéologiques?, actes du colloque international du 13 au 15 mai 2009, université Toulouse II - Le Mirail, P@lethnologie,3,p. 131-168.

Baffier D., Julien M.

1990: «L'outillage en os des niveaux châtelperroniens d'Arcy-sur-Cure», in FARIZY C. (éd.), Paléolithique moyen récent et Paléolithique supérieur ancien en Europe. Ruptures et transitions : examen critique des documents archéologiques, Nemours, actes du colloque international, 9-11 mai 1988, Mémoires du Musée de Préhistoire d'Île-deFrance, 3, APRAIF, Paris, p. 329-334

Baillet M.

2010: Étude fonctionnelle des grattoirs de Canaule II (Creysse, Dordogne), site châtelperronien de plein air en Bergeracois, Mémoire de Master II, Université de Bordeaux I, Talence, inédit, $78 \mathrm{p}$.

Baillet M., Bachellerie F., Bordes J.-G. 2014: «Enquête autour d'un outil : approche techno-économique, fonctionnelle et expérimentale des grattoirs châtelperroniens de Canaule II (Creysse, Dordogne, France)», Paléo, 25, p. 7-36.

BANKs W.

2015: 'Constructing chronologies for the late Middle Paleolithic and Upper
Paleolithic: limitations and means to overcome them', World Archaeology, 47 (4), p. 585-600.

Barsness L.

1977: The Bison in Art. A graphic Chronicle of the American Bison, Amon Carter Museum of Western Art, Northland Press, 142 p.

Bar-Yosef O., Bordes J.-G.

2010: "Who were the makers of the Châtelperronian culture?', Journal of Human Evolution, 59(5), p. 586-593.

BeYRIES S.

2002: «Le travail du cuir chez les Tchouktches et les Athapaskans : Implications ethno-archéologiques», in Audouin-Rouzeau F., Beyries S. (éds.), Le travail du cuir de la préhistoire à nos jours, XXII e rencontres internationales d'archéologie et d'histoire d'Antibes, 18-20 octobre 2001, édition APDCA, Antibes, p. 11-12.

BINFORD L.R.

1981 : Bones: Ancient Men and Modern Myths, New York Academic Press, 320 p.

Blaser F., Bourguignon L., Sellami F., Rios J.

2012: «Une série lithique à composante Laminaire dans le Paléolithique moyen du Sud-Ouest de la France : le site de Cantalouette 4 (Creysse, Dordogne, France)», Bulletin de la Société préhistorique française, 109 (fasc. 1), p. 5-33.

Bodu $\mathbf{P}$.

1990: «L'application de la technique des remontages à l'étude du matériel lithique des premiers niveaux Châtelperroniens de la grotte du Renne à Arcy-sur-Cure (Yonne)", in FArizy C. (éd.), Paléolithique moyen récent et Paléolithique supérieur ancien en Europe. Ruptures et transitions : examen critique des documents archéologiques, Nemours, actes du colloque international, 9-11 mai 1988 , Mémoires du Musée de Préhistoire d'Île-de-France, 3, APRAIF, Paris, p. 309-312.

Bodu P., Chehmana L., Debout G.

2007: «Le Badegoulien de la moitié nord de la France. Un état des connaissances», Bulletin de la Société préhistorique française, 104 (4), p. 661-679. 
Bodu P., Mevel L.

2008: "Enquête autour des lames tranchantes de l'Azilien ancien. Le cas du niveau inférieur du Closeau (RueilMalmaison, Hauts-de-Seine, France)», L'Anthropologie, 112, p. 509-543.

Bodu P., Bignon O., Dumarcay G.

2011: «Le gisement des Bossats à Ormesson, région de Nemours (Seine-et-Marne) : un site gravettien à faune dans le Bassin parisien ", in Goutas N., Klaric L., Pesesse D., Guillermin P. (éds.), À la recherche des identités gravettiennes: actualités, questionnements et perspectives, actes de la table-ronde sur le Gravettien en France et dans les pays limitrophes, Aix-en-Provence (6-8 octobre 2008), Société préhistorique française, Mémoire LII, Paris, p. 259-272.

Bodu P., Renard C.

2013a: "L'ancien” Solutréen du Bassin parisien : quelques observations récentes », in Bodu P., Chehmana L., Klaric L., Mevel L., Soriano S., Teyssandier N. (dir.), Le Paléolithique supérieur ancien de l'Europe du NordOuest (35000-15000 BP) : réflexions et synthèses à partir d'un projet collectif de recherche sur le Paléolithique supérieur ancien du Bassin parisien, actes du colloque, Sens, avril 2009, Société préhistorique française, Mémoire LVI, Paris, p. 117-141.

Bodu P., Bon F., Teyssandier N., Paris C. 2013b: «L'Aurignacien et les faciès à pièces carénées entre Yonne et Yvelines», in Bodu P., Chehmana L., Klaric L., Mevel L., Soriano S., Teyssandier N. (dir.), Le Paléolithique supérieur ancien de l'Europe du Nord-Ouest $(35000-15000 \mathrm{BP})$ : réflexions et synthèses à partir d'un projet collectif de recherche sur le Paléolithique supérieur ancien du Bassin parisien, actes du colloque, Sens, avril 2009, Société préhistorique française, Mémoire LVI, Paris, p. 37-60.

Bodu P., Chehmana L., Klaric L., Mevel L., Soriano S., Teyssandier N. (Dir.) 2013c: Le Paléolithique supérieur ancien de l'Europe du Nord-Ouest (35000-15000 $B P)$ : réflexions et synthèses à partir d'un projet collectif de recherche sur le Paléolithique supérieur ancien du Bassin parisien, actes du colloque, Sens, avril 2009, Société préhistorique française, Mémoire LVI, Paris, 516 p.

Bodu P., Dumarcay G., Naton H.-G. avec la collaboration de Ballinger M. et de TheryParisot I.

2014a: «Un nouveau gisement solutréen en Île-de-France, le site des Bossats à
Ormesson (Seine-et-Marne)», Bulletin de la Société préhistorique française, 111 (2), p. 225-254.

Bodu P., Salomon H., Leroyer M., Naton H.-G., Lacarriere J., Dessoles M. 2014b: An open-air site from the recent middle Palaeolithic in the Paris Basin (France): Les Bossats at Ormesson (Seine-et-Marne), Quaternary International, 331, p. 39-59.

Bordes J.G.

2002: Les interstratifications Châtelperronien/Aurignacien $d u$ Roc de Combe et du Piage (Lot, France) : analyse taphonomique des industries lithiques, implications archéologiques, Thèse de Doctorat, Université de Bordeaux I, inédit, 365 p.

Bordes J.-G., Teyssandier N.

2011: 'The Upper Paleolithic Nature of the Châtelperronian in Southwestern France: Archeostratigrapic and Lithic Evidence', Quaternary International, 246 (1-2), p. 382-388.

Breuil $\mathbf{H}$.

1911: «Études de morphologie paléolithique II. - L'industrie de la grotte de Châtelperron (Allier) et d'autres gisements similaires $»$, Revue de l'École d'Anthropologie de Paris, 21, p. 29-40.

Caron F., D'errico F., Del Moral P., SAntos F., J. ZiLhão J.

2011: «The Reality of Neandertal Symbolic Behavior at the Grotte du Renne, Arcysur-Cure, France», PLoS ONE, 6 (6), p. e21545.

Cheynier A.

1962: Le Cirque-de-la-Patrie à Nemours (Seine-et-Marne), Société préhistorique française, Mémoire VI, Paris, $195 \mathrm{p}$.

Connet N.

2002: Le Châtelperronien : Réflexions sur l'unité techno-économique de l'industrie lithique. L'apport de l'analyse diachronique des industries lithiques des couches châtelperroniennes de la grotte du Renne à Arcy-sur-Cure (Yonne), Thèse de Doctorat, Université de Lille I, inédit, 685 p.

\section{Cretin C.}

1996: «Vers une nouvelle perception du Badegoulien des Jamblancs : Premiers éléments techno-économiques », Paléo, 8, p. 243-268.

Daniel R.

1930: «Étude sur les différentes industries lithiques de la station du "Beauregard" près Nemours (Seine-et-Marne)»,
Bulletin de l'Association des naturalistes de la vallée du Loing, 13 (1-2), p. 67-83.

Daniel M. et Daniel R.

1953: «Les gisements préhistoriques de la vallée du Loing», L'Anthropologie, 57, p. 209-239.

David F., Connet N., Girard M., Lhomme V., Miskovsky J.C., RoblinJouve A.

2001: «Le Châtelperronien de la grotte du Renne à Arcy-sur-Cure (Yonne). Données sédimentologiques et chronostratigraphiques», Bulletin de la Société préhistorique française, 98 (2), p. 207-230.

Dayet L., D'errico F., Garcia-Moreno R. 2014: 'Searching for consistencies in Châtelperronian pigment use', Journal of Archaeological Science, 44, p. 180-193.

Delarue R., Vignard E.

1959: «L'Aurignaco-Périgordien des Gros Monts, Bis et Ter, dans les bois de Beauregard de Nemours », Bulletin de la Société préhistorique française, 56 (7-8), p. 166-171.

Denizot G.

1971: Carte géologique de la France, 1:50000, $\mathrm{n}^{\circ} 329$, Château-Landon, Orléans, BRGM.

DePaepe P.

2007: Le Paléolithique moyen de la vallée de la Vanne (Yonne, France) : matières premières, industries lithiques et occupations humaines, Société préhistorique française, Mémoire XLI, Paris, 298 p.

D'errico F., Zilhão J., Julien M., Baffier D., Pelegrin J.

1998: « Neanderthal Acculturation in Western Europe? A Critical Review of the Evidence and its Interpretation', Current Anthropology, 39 (2), p. S1-S44.

D'errico F., Julien M., Liolios D., Baffier D., Vanhaeren M.

2001: «Les poinçons en os des couches châtelperroniennes et Aurignaciennes de la grotte du Renne (Arcy-sur-Cure, Yonne) : comparaisons technologiques, fonctionnelles et décor», in Bodu P., Constantin C. (dir.), Approches fonctionnelles en préhistoire, actes du $X X V^{e}$ Congrès Préhistorique de France (Nanterre), Société Préhistorique Française, Paris, p. 45-66.

Discamps E., Jaubert J., Bachellerie F. 2011: 'Human choices and environmental constraints: deciphering the vari- 
ability of large game procurement from Mousterian to Aurignacian times (MIS 5-3) in southwestern France', Quaternary Science Reviews, 30 (19), p. 2755-2775.

Discamps E., Soulier M.-C., Bachellerie F., Bordes J.-G., Castel J.-C., Morin E.

2014: «Des faunes et des hommes : interactions entre environnements et cultures à la fin du Paléolithique moyen et au début du Paléolithique supérieur dans le Sud-Ouest de la France», in Thiébaut C., Costamagno S., Claud E. (éds.), Transitions, ruptures et continuité en Préhistoire, XXVIIe congrès préhistorique de France, BordeauxLes Eyzies, 31 mai-5 juin 2010, Société préhistorique française, Mémoire XXXI, p. 299-314.

Discamps M., Gravina B., Teyssandier N. 2015: 'In the eye of the beholder: contextual issues for Bayesian modelling at the Middle-to-Upper Palaeolithic transition', World Archaeology, 47 (4), p. 601-621.

Dutkiewicz E., Floss H.

2015: «La grotte de la Verpillière I à Germolles, site de référence paléolithique en Bourgogne méridionale. Historique des 150 ans de recherche», La Physiophile, 162, p. 12-32.

Enloe, J. G.

2012: Neanderthals, bears and hyenas, oh my! competition for exclusive use of space, Journal of Taphonomy, 10 (3-4), p. 193-195.

FARIZY C.

1990 : «Du Moustérien au Châtelperronien à Arcy-sur-Cure : un état de la question ", in Farizy C. (éd.), Paléolithique moyen récent et Paléolithique supérieur ancien en Europe. Ruptures et transitions : examen critique des documents archéologiques, Nemours, actes du colloque international, 9-11 mai 1988, Mémoires du Musée de Préhistoire d'Île-de-France, 3, APRAIF, Paris, p. 281-289.

Flas D.

2014: «Les industries à pointes foliacées dans les régions septentrionales de l'Europe », in Otte M. (éd.), Néandertal/ Cro-Magnon : La rencontre. Civilisations et Cultures, Éditions Errance, Paris, p. 97-122.

Floss H., Dutkiewicz E., Frick Jens A., Hoyer C.

2013: «Le Paléolithique supérieur ancien en Bourgogne du sud», in Bodu P., Chehmana L., Klaric L., Mevel L.,
Soriano S., Teyssandier N. (dir.), Le Paléolithique supérieur ancien de l'Europe du Nord-Ouest (35000$15000 \mathrm{BP})$ : réflexions et synthèses à partir d'un projet collectif de recherche sur le Paléolithique supérieur ancien $d u$ Bassin parisien, actes du colloque, Sens, avril 2009, Société préhistorique française, Mémoire LVI, Paris, p. 331-350.

Gilchrist R., Mytum C.

1986: «Experimental archaeology and burnt animal bone from archaeological sites », Circaea, 4, p. 29-38.

Grayson D. K., Delpech F.

2003: 'Ungulates and the middle-to-upper Paleolithic transition at Grotte XVI (Dordogne, France)', Journal of Archaeological Science, 30 (12), p. 1633-1648.

Grayson, D. K., Delpech, F.

2008: 'The large mammals of Roc de Combe (Lot, France): the Châtelperronian and Aurignacian assemblages', Journal of anthropological archaeology, 27 (3), p. $338-362$.

Grigoletto F., Ortega I., Rios J., Bourguignon L.

2008: «Le Châtelperronien de Vieux Coutets - premiers éléments de réflexion », in JAUBERT J., BORDES J-G., Ortega I. (éds.), Les sociétés $d u$ Paléolithique dans un grand Sud-ouest : nouveaux gisements, nouveaux résultats, nouvelles méthodes, Séances de la Société préhistorique française, 24-25 novembre 2006, Bordeaux, Mémoire XLVII, Paris p. 245-259.

Higham T., Jacobi R., Julien M., David F., Basell L., Wood R., Davies W., Bronk RAMSEY C.

2010: 'Chronology of the Grotte du Renne (France) and Implications for the Context of Ornaments and Human Remains within the Châtelperronian', Proceeding of the National Academy of Science, 107 (47), p. 20234-20239.

Higham T., Douka K., Wood, R., Ramsey C. B., Brock F., Basell L., Camps M., Arrizabalaga A., Baena J., Barroso-Ruíz C., Bergman C., Boitard C., Boscato P., Caparrós M., Conard N. J., Draily C., Froment A., Galván B., Gambassini P., GarciaMoreno A., Grimaldi S., Haesaerts P., Holt B., Iriarte-Chiapusso M.-J., Jelinek A., Jordá Pardo J. F., MaílloFernández J.-M, Marom A., Maroto J., Menéndez M., Metz L., Morin E., Moroni A., Negrino F., Panagopoulou E., Peresani M., Pirson S., de la Rasilla M., Riel-Salvatore J., Ronchitelli A.,
Santamaria D., Semal P., Slimak L., Soler J., Soler N., Villaluenga A., Pinhasi R., Jacobi R.

2014: 'The timing and spatiotemporal patterning of Neanderthal disappearance', Nature, 512, p. 306-309.

Hinguant S., Biard M.

2013: «Le Paléolithique supérieur ancien de la Vallée de l'Erve (Mayenne) : un état des connaissances », in Bodu P., Chehmana L., Klaric L., Mevel L., Soriano S., Teyssandier N. (Dir.), Le Paléolithique supérieur ancien de l'Europe du Nord-ouest (3500015000 BP) : Réflexions et synthèses à partir d'un projet collectif de recherche sur le Paléolithique supérieur ancien du Bassin parisien, Sens, avril 2009, Société préhistorique française, Mémoire LVI, Paris, p. $239-250$.

Hublin J.-J., Spoor F., Braun M., Zonneveld F., Condemi S.

1996: «A Late Neanderthal Associated with Upper Palaeolithic Artefacts », Nature, 381 (6579), p. 224-226.

Hublin J.-J., Talamo S., Julien M., David F., Connet N., Bodu P., VANDERmeersch B., RichaRds M.-P.

2012: 'Radiocarbon dates from the Grotte du Renne and Saint-Césaire support a Neandertal Origin for the Châtelperronian', Proceedings of the National Academy of Sciences, 109(46), p. $18743-18748$.

Lacarriere J., Bodu P., Dumarçay G., Goutas N., Julien M.A., LeJay M., Peschaux C., Naton H.-G., TheryParisot I., Vasiliu L.

2015: 'Les Bossats (Ormesson, Paris basin, France): A new early Gravettian bison processing camp', Quaternary International, 359-360, p. 520-534.

Lanos P., Philippe A., Lanos H., Dufresne P.

2015: ChronoModel: Chronological Modelling of Archaeological Data using Bayesian Statistics. (Version 1.5.0). Available from http://www.chronomodel.fr.

\section{LENOIR M.}

1983: Le Paléolithique des basses vallées de la Dordogne et de la Garonne, Thèse de Doctorat d'état, Université de Bordeaux 1, Talence, inédit, 2 t., $702 \mathrm{p}$.

Leroi-Gourhan A.

1958: «Étude des restes humains fossiles provenant des Grottes d'Arcy-surCure», Annales de Paléontologie, 44, p. $87-148$ 
Leroi-Gourhan A. et Leroi-Gourhan A.

1964: «Chronologie des grottes d'Arcy-surCure (Yonne)», Gallia Préhistoire, 7, $64 \mathrm{p}$.

Letourneux C.

2003: Devine qui est venu dîner à Brassempouy? Approche taphonomique pour une interprétation archéozoologique des vestiges osseux de l'Aurignacien ancien de la grotte des Hyènes (Brassempouy, Landes), Thèse de Doctorat, Université PanthéonSorbonne, Paris I, 603 p.

LÉVÊque F., VANDERMEersch B.

1980: «Découverte de restes humains dans un niveau Castelperronien à Saint-Césaire (Charente-Maritime)», Comptes Rendus de l'Académie des Sciences, Paris, Série D 291, p. 187-189.

Lévêque F., Backer A.M., Guilbaud M.

1993: Context of a Late Neandertal: Implications of Multidisciplinary Research for the Transition to Upper Paleolithic Adaptations at SaintCésaire, Charente-Maritime, France. Madison, WI: Prehistory Press.

Lhomme V., David F., Thiebaut C.

2005: «Les industries de la fin du Paléolithique moyen de la grotte du bison à Arcy-sur-Cure (Yonne)». in Molines N., Moncel M.-H., Monnier. L. (éds.), Données récentes sur les modalités de peuplement et sur le cadre chronostratigraphique, géologique et paléogéographique des industries du Paléolithique inférieur et moyen en Europe, actes du colloque international de Rennes, 22-25 septembre 2003, British Archaeological Reports, 1364 (I), p. 479-495.

Malgarini R., Mevel L., Bereiziat G., Bodu P., Debout G., Cupillard C., Carquigny N.

2017: "Les faciès du Magdalénien moyen dans l'Est de la France : confrontation et discussion des industries osseuses et lithiques ", in BOURdier C., Chehmana L., Malgarini R., Poltowicz-Bobak M. (dir.), L'essor $d u$ Magdalénien. Aspects culturels, symboliques et techniques des faciès à Navettes et à Lussac-Angles, actes de la séance de la Société préhistorique française de Besançon, 17-19 octobre 2013, Séances de la Société préhistorique française, 8 , Paris, 2016, p. 139-156.

Mathis F., Bodu P., Dubreuil O., SAlomon H.

2014: 'PIXE identification of the provenance of ferruginous rocks used by Neanderthals', Nuclear Instruments and Methods in Physics Research Section B: Beam Interactions with Materials and Atoms, 331, p. 275-279.

Mellars P.

2010: 'Neanderthal symbolism and ornament manufacture: The bursting of a bubble?', Proceedings of the National Academy of Science USA, 107 (47), p. 20147-20148.

Morala A.

1993: 'Technologie lithique du Magdalénien ancien de l'abri casserole (Les Eyziesde-Tayac, Dordogne)', Paléo, 5, p. 193-208.

Morin E.

2006: «Late Pleistocene population interaction in Western europe and modern human origins: new insights based on the faunal remains from Saint-Césaire, southwestern France», Bulletin de la Société préhistorique française, 103 (1), p. 186-188.

OLSEN S.J.

1960: 'Post-Cranial Skeletal Characters of Bison and Bos', Papers of the Peabody Museum of Archaeology and Ethnology, 35 (4), p. 3-15.

Parat A.

1904: «Les grottes de la vallée de l'Yonne. XXXVI. La grotte de la Roche-auLoup et les grottes de Merry-surYonne, Brosses, Chatel-Censoir, Crain, Festigny, Druyes », Bulletin de la Société des Sciences historiques et naturelles de l'Yonne, 2ème sem., 68 p., 1 pl.

Patou-Mathis M.

2005: «Comportement de subsistance des Néandertaliens du niveau châtelperronien de Saint-Césaire (CharenteMaritime)», Antropologia-Arkeologia, 57, p. 197-204.

Pelegrin J.

1995: Technologie lithique : le Châtelperronien de Roc-de-Combe (Lot) et de la Côte (Dordogne). Cahiers du Quaternaire, 20, éditions du CNRS, $297 \mathrm{p}$.

2000: « Les techniques de débitage laminaire au Tardiglaciaire : critères de diagnose et quelques réflexions », in VALENTIN B. Bodu P., Christensen M. (dir.), L'Europe centrale et septentrionale au Tardiglaciaire, actes de la table-ronde internationale, Nemours, 14-16 mai 1997, Musée de Préhistoire d'Île-deFrance, 7, APRAIF, Nemours, p. 73-86.

Pelegrin J., Soressi M.

2007: «Le Châtelperronien et ses rapports avec le Moustérien», in Vandermeersch B., Maureille B. (éds.), Les Néandertaliens. Biologie et cultures, éditions du CTHS, documents préhistoriques, 33, Paris, p. 283-296.

Pirson S., Di Modica K.

2011: "Position chronostratigraphique des productions lithiques du Paléolithique ancien en Belgique : un état de la question ", in Toussain M., Di Modica K., Pirson S. (éds.), Le Paléolithique moyen en Belgique, Mélanges Margueritte Urlix-Closset, E.R.A.U.L, 128, p. 105-128.

Pirson S., Flas D., Abrams G., Bonjean D., Court-Picon M., Di Modica K., Draily C., Damblon F., Haesaerts P., Miller R., Rougier H., Toussaint M. \& Semal, P.

2012: 'Chronostratigraphic context of the Middle to Upper Palaeolithic transition: Recent data from Belgium', Quaternary International, 259, p. 78-94.

Plisson H., Schmider B.

1990: «Étude préliminaire d'une série de pointes de Châtelperron de la Grotte du Renne à Arcy-sur-Cure», in FARIZY C. (éd.), Paléolithique moyen récent et Paléolithique supérieur ancien en Europe. Ruptures et transitions : examen critique des documents archéologiques, Nemours, actes du colloque international, 9-11 mai 1988, Mémoires du Musée de Préhistoire d'Île-deFrance, 3, APRAIF, Paris, p. 313-318.

Poplin F.

1981: Rapport sur la campagne de fouilles préhistoriques de 1981 à la Roche-auLoup, Commune de Merry-sur-Yonne (Yonne) ; Le sondage du Mahaleb, inédit, Service régional de l'Archéologie de Bourgogne, $49 \mathrm{p}$.

1982: Rapport sur la campagne de fouilles préhistoriques de 1982 à la Rocheau-Loup, Commune de Merry-surYonne (Yonne); Le sauvetage de l'entrée, le sondage du cachot, inédit, Service régional de l'Archéologie de Bourgogne, $42 \mathrm{p}$.

1983a : Rapport sur la campagne de fouilles préhistoriques de 1983 à la Roche-auLoup (Merry-sur-Yonne); Le sauvetage de l'entrée, le sondage du fond, inédit, Service régional de l'Archéologie de Bourgogne, $55 \mathrm{p}$.

1983b: «Nouvelles fouilles préhistoriques à la Roche-au-Loup (Merry-sur-Yonne)», Bulletin de la Société des Sciences de l'Yonne, 114, p. 23-45.

1984: Rapport sur la campagne de fouilles préhistoriques de 1984 à la Roche au Loup (Merry-sur-Yonne) ; La coupe du cône de déblais, la brèche du couloir d'entrée, le diverticule à droite au fond de la grande salle, la fouille du fond, inédit, Service régional de l'Archéologie de Bourgogne, $20 \mathrm{p}$. 
1985 : Les fouilles préhistoriques de Merrysur-Yonne, rapport 1985, inédit, Service régional de l'Archéologie de Bourgogne, $107 \mathrm{p}$.

1986a: Les fouilles préhistoriques de Merry-sur-Yonne, grotte de la Rocheau-Loup, campagne 1986, inédit, Service régional de l'Archéologie de Bourgogne, $111 \mathrm{p}$.

1986b: «La légende de l'hippopotame de la Roche-au-Loup », Bulletin de la Société préhistorique française, 82,8 , p. $232-235$.

1986c: «Découverte et utilisation probable de galène dans le Châtelperronnien de Merry-sur-Yonne (Yonne)», Bulletin de la Société préhistorique française, 83, p. 132.

1987: Les fouilles préhistoriques de Merry-sur-Yonne, grotte de la Rocheau-Loup, campagne 1987, inédit, Service régional de l'Archéologie de Bourgogne, $61 \mathrm{p}$.

1988: «Aux origines néandertaliennes de l'Art. Matière, forme, symétrie. Contribution d'une galène et d'un oursin fossile taillé de Merry-sur-Yonne (France)», L'Homme de Néandertal, vol. 5. La Pensée, ERAUL, 32, Liège, p. 109-116.

Reimer P.J., Bard E., Bayliss A., Warren Beck J., Blackwell P.G., Ramsey C.B., Buck C.E., Cheng H., Edwards R.L., Friedrich M., Grootes P.M., Guilderson R.P., Haflidason H., HaJda I., Hatté C., Heaton T.J., Hoffmann D.L., Hogg A.G., Hughen K.A., Felix Kaiser K., Kromer B., Manning S.W., NiU M., Reimer R.W., Richards D.A., Marian Scott E., Southon J.R., Staff R.A., Turney C.S.M., Van Der Plicht J.

2013: IntCal13 and Marine13 Radiocarbon Age Calibration Curves 0-50,000 Years cal BP, Radiocarbon, 55 (4), p. 18691887.

Revillon S., Tuffreau A.

1994: Les industries laminaires au Paléolithique moyen, actes de la table-ronde, Villeneuve d'Ascq, 1991, Éditions du CNRS, Paris, 193 p.

Rios-Garaizar J.

2008: Nivel IX (Chatelperroniense) de Labeko Koba (Arrasate-Gipuzkoa): gestión de la industria lítica y función del sitio, Munibe (AntropológicaArkeologia), 59, p. 25-46.

Roussel M.

2011 : Normes et variations de la production lithique durant le Châtelperronien. La séquence de la Grande-Roche-de-laPlématrie à Quincay (Vienne), Thèse de Doctorat, Université de Paris Ouest Nanterre-la-Défense, inédit, 554 p.
Roussel M., Soressi M.

2013: «Une nouvelle séquence du Paléolithique supérieur ancien aux marges sud-ouest du Bassin parisien : Les Cottés ", in Bodu P., Chehmana L., Klaric L., Mevel L., Soriano S., Teyssandier N. (dir.), Le Paléolithique supérieur ancien de l'Europe $d u$ Nord-Ouest (35000-15000 BP) : réflexions et synthèses à partir d'un projet collectif de recherche sur le Paléolithique supérieur ancien $d u$ Bassin parisien, actes du colloque, Sens, avril 2009, Société préhistorique française, Mémoire LVI, Paris, p. 283-298.

2014: «Le Châtelperronien», in Отте M. (éd.), Néandertal/Cro-Magnon : La rencontre. Civilisations et Cultures, Éditions Errance, Paris, p. 31-59.

Roussel M., Soressi M., Hublin J.-J.

2016: 'The Châtelperronian conundrum: Blade and bladelet lithic technologies from Quincay, France', Journal of Human Evolution, 95, p. 13-32.

SALOMON H.

2009: Les matières colorantes au début du Paléolithique supérieur : sources, transformations et fonctions, Thèse de Doctorat, Université de Bordeaux 1, inédit, $413 \mathrm{p}$.

2017 à paraître: Les matières colorantes, in Julien M. (éd.), le Châtelperronien de la Grotte du Renne à Arcy-sur-Cure. Les fouilles d'André Leroi-Gourhan, Paléo, $\mathrm{n}^{\circ}$ spécial.

SCANDIUZZI R.

2008: Les Tambourets, un gisement châtelperronien de plein air au seuil des Petites Pyrénées. Étude de l'industrie lithique. Fouilles H.M. Bricker (1973-1975-1980, Couladère, Haute-Garonne), Mémoire de Master II, Université Toulouse II Le Mirail, inédit, 131 p.

SCHMider B.

1971: Les industries du Paléolithique Supérieur en Île-de-France. $6^{\circ}$ Suppl. à Gallia Préhistoire, Éditions du CNRS, Paris, 243 p.

Schmider B., Roblin-Jouve A.

2008: Le massif de Fontainebleau au Paléolithique supérieur. Les grands sites d'habitat préhistorique, évolution des cultures et des paysages, ERAUL, 120, Liège, 65 p.

Soulier M.-C.

2013: "Entre alimentaire et technique»: L'exploitation animale aux débuts du Paléolithique supérieur. Stratégies de subsistance et chaîne opératoire de traitement du gibier à Isturitz, La Quina Aval, Roc de Combe et Les
Abeilles, Thèse de Doctorat, Université de Toulouse II, inédit, $756 \mathrm{p}$.

Stocker T.F., Johnsen S.J.

2003: "A minimum thermodynamic model for the bipolar seesaw», Paleoceanography, 18 (4), p. 108.

Szmidt C., Brou L., Jaccottey L.

2010: « Direct radiocarbon (AMS) dating of split-based points from the (Proto) Aurignacian of Trou de la Mère Clochette, Northeastern France. Implications for the characterization of the Aurignacian and the timing of technical innovations in Europe', Journal of Archaeological Science, 37, p. 3320-3337.

TABORIN Y.

1990: "Les prémices de la parure », in FARIZY C. (éd.), Paléolithique moyen récent et Paléolithique supérieur ancien en Europe. Ruptures et transitions : examen critique des documents archéologiques, Nemours, actes du colloque international, 9-11 mai 1988, Mémoires du Musée de Préhistoire d'Île-de-France, 3, APRAIF, Paris, p. 335-344.

Tostevin G.B.

2007: 'Social Intimacy, Artefact Visibility, and Acculturation Models of NeanderthalModern Human Interaction', in Mellars P., Boyle K., Bar-Yosef O., Stringer C. (éds.), Rethinking the Human Revolution: New Behavioural and Biological Perspectives on the Origins and Dispersal of Modern Humans, p. 341-357.

VACHÉ C.

1932: «Une pointe type Châtelperron dans le niveau aurignacien du Beauregard », Bulletin del'Association des Naturalistes de la Vallée du Loing, p. 86-87.

Vande Walle $\mathbf{H}$.

2003: " La production des outils au Paléolithique moyen : comparaison diachronique des occupations de Riencourt-lès-Bapaume (Pas-deCalais, France) », Paléo, 15, p. 169-194.

\section{VANDERMEERSCH B.}

1984: « À propos de la découverte du squelette Néandertalien de SaintCésaire ", Bulletins et Mémoires de la Société d'Anthropologie de Paris, 14 (1-3), p. 191-196.

Vignard E., Delarue R.

1960: « Le protomagdalénien I du bois des Chênes sur la platière des Beauregards près de Nemours (Seine-et-Marne) ", Bulletin de la Société préhistorique française, 57, p. 607-620. 
Welker F., Hadjinjak M., Talamo S., Jaouen K., Dannemamm M., David F., Julien M., Meyer M., Kelso J., Barnes I., Brace S., Kamminga P., Fisher R., Kessler B.M., Stewart J.R., Pä̈̈вo S., Collins M., Hublin J.-J.

2016: 'Paleoproteomic evidence identifies archaic hominins associated with the Châtelperronian at the Grotte du Renne', Proceedings of the National Academy of Sciences USA, 113 (40), p. 11162-11167.
WhITE R.

2001: 'Personal Ornaments from the Grotte du Renne at Arcy-sur-Cure', Athena Review, 2 (4), p. 41-46.

Zilhão J., D'errico F., Bordes J.-G., Lenoble A., Texier J.-P., Rigaud J.-P.

2006: 'Analysis of Aurignacian interstratification at the Châtelperronian-type site and implications for the behavioral modernity of Neandertals', Proceedings of the National Academy of Sciences USA, 103 (33), p. 12643-12648.
2007: 'Grotte des Fées (Châtelperron) : History of Research, Stratigraphy, Dating, and Archaeology of the Châtelperronian type-site', PaleoAnthropology, 1-4

Zollikofer, C., Ponce De Leon, M., VANDERmeersch, B., LeVeque, F.

2002: 'Evidence for interpersonal violence in the St. Césaire Neanderthal', Proceedings of the National Academy of Sciences USA, 99 (9), p. 6444-6448. 